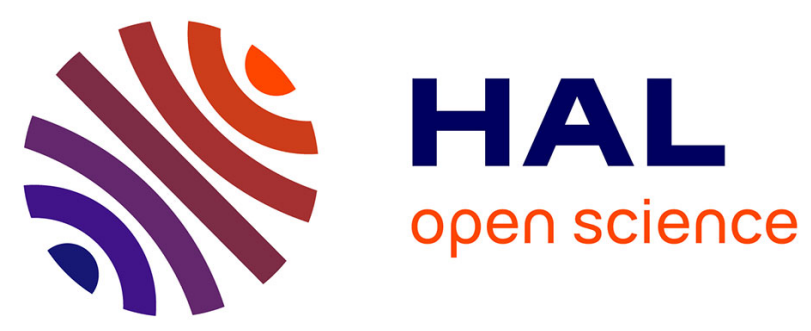

\title{
Prediction of necking in HCP sheet metals using a two-surface plasticity model
}

\author{
Mohamed Yassine Jedidi, Mohamed Ben Bettaieb, Farid Abed-Meraim, \\ Mohamed Taoufik Khabou, Anas Bouguecha, Mohamed Haddar
}

\section{- To cite this version:}

Mohamed Yassine Jedidi, Mohamed Ben Bettaieb, Farid Abed-Meraim, Mohamed Taoufik Khabou, Anas Bouguecha, et al.. Prediction of necking in HCP sheet metals using a two-surface plasticity model. International Journal of Plasticity, 2020, 128, pp.102641. 10.1016/j.ijplas.2019.102641 hal02517380

\author{
HAL Id: hal-02517380 \\ https://hal.science/hal-02517380
}

Submitted on 24 Mar 2020

HAL is a multi-disciplinary open access archive for the deposit and dissemination of scientific research documents, whether they are published or not. The documents may come from teaching and research institutions in France or abroad, or from public or private research centers.
L'archive ouverte pluridisciplinaire HAL, est destinée au dépôt et à la diffusion de documents scientifiques de niveau recherche, publiés ou non, émanant des établissements d'enseignement et de recherche français ou étrangers, des laboratoires publics ou privés. 


\title{
Prediction of necking in HCP sheet metals using a two-surface plasticity model
}

\author{
M.Y. Jedidi ${ }^{1,3}$, M. Ben Bettaieb ${ }^{1,2^{*}}$, F. Abed-Meraim ${ }^{1,2}$, M.T. Khabou ${ }^{3}$, A. Bouguecha ${ }^{3}$, M. \\ Haddar $^{3}$ \\ ${ }^{1}$ Université de Lorraine, CNRS, Campus Arts et Métiers de Metz, LEM3, F-57000, France \\ ${ }^{2}$ DAMAS, Laboratory of Excellence on Design of Alloy Metals for low-mAss Structures, Université \\ de Lorraine, France \\ ${ }^{3}$ Laboratory of Mechanics, Modeling and Production (LA2MP), National School of Engineers of Sfax \\ - Sfax - Tunisia \\ *Corresponding author: Mohamed.BenBettaieb@ensam.eu
}

\begin{abstract}
In the present contribution, a two-surface plasticity model is coupled with several diffuse and localized necking criteria to predict the ductility limits of hexagonal closed packed sheet metals. The plastic strain is considered, in this two-surface constitutive framework, as the result of both slip and twinning deformation modes. This leads to a description of the plastic anisotropy by two separate yield functions: the Barlat yield function to model plastic anisotropy due to slip deformation modes, and the Cazacu yield function to model plastic anisotropy due to twinning deformation modes. Actually, the proposed two-surface model offers an accurate prediction of the plastic anisotropy as well as the tension-compression yield asymmetry for the material response. Furthermore, the current model allows incorporating the effect of distortional hardening resulting from the evolution of plastic anisotropy and tension-compression yield asymmetry. Diffuse necking is predicted by the general bifurcation criterion. As to localized necking, it is determined by the Rice bifurcation criterion as well as by the Marciniak \& Kuczynski imperfection approach. To apply both bifurcation criteria, the expression of the continuum tangent modulus associated with this constitutive framework is analytically derived. The set of equations resulting from the coupling between the Marciniak \& Kuczynski approach and the constitutive relations is solved by developing an efficient implicit algorithm. The numerical implementation of the two-surface model is assessed and validated through a comparative study between our numerical predictions and several experimental results from the literature. A sensitivity study is presented to analyze the effect of some mechanical parameters on the
\end{abstract}


prediction of diffuse and localized necking in thin sheet metals made of HCP materials. The effect of distortional hardening on the onset of plastic instability is also investigated.

Keywords: two-surface plasticity model; hexagonal closed packed; plastic anisotropy; strength asymmetry; plastic instability; forming limit diagram.

\section{Introduction}

Due to their interesting physical and mechanical properties, hexagonal closed packed (HCP) materials, such as titanium, magnesium and beryllium alloys, are widely used in aircraft, aerospace, automotive, computer and mobile device industries, as alternatives to conventional materials, such as steel or aluminum alloys. Indeed, HCP materials are characterized by their high tensile strength to density ratio (Li et al., 2017), high corrosion resistance (Lide, 2005), and high crack and fatigue resistance (Moiseyev, 2006). To better optimize the use of HCP materials in new industrial applications, their mechanical behavior should be accurately investigated. In this field, the modeling of physical mechanisms and modes that lead to plastic deformation and anisotropy in this class of materials has been getting growing attention from the scientific community (Cazacu and Barlat, 2004; Cazacu et al., 2006; Fan et al., 2018; Paramatmuni and Kanjarla, 2018; Kondori et al., 2019...). Several experimental investigations show that plastic deformation in HCP materials is mainly due to the activation of twinning and slip modes. These investigations also revealed that twinning mode results in tension-compression strength asymmetry, known as strength differential effect. Furthermore, the small number of slip systems in HCP materials (typically 3 to 6 slip systems versus 24 slip systems in BCC materials) leads to strong initial and evolving plastic anisotropy. In spite of the large number of works devoted to the study of their plastic anisotropy, experimental and theoretical investigations of HCP material ductility are still seldom, and often not very extensive (Chen and Hang, 2003; Chang et al., 2007; Lee et al., 2008; Steglich et Jeong, 2016). Though, during the design stage of a mechanical system, the forming capabilities of thin components are crucial to determine their behavior and service lifetime. In this context, a decision support tool that would provide relevant information from numerical prediction of formability limits should be developed. Towards this perspective, we have coupled an elaborate constitutive framework, which is capable of describing the evolution of the mechanical variables during deformation of $\mathrm{HCP}$ materials, with several plastic instability criteria. This coupling is used to predict the onset of diffuse and localized necking and to represent the predicted necking limit strains in terms of forming limit diagrams (FLDs).

- Constitutive modeling of HCP materials: to accurately model the mechanical behavior of HCP materials, several multiscale strategies have been set up in the literature (Lebensohn and Tomé, 1993; Kalidindi, 1998; Staroselsky and Anand, 1998; Wang et al., 2010; Wang et al., 2011; Song and Castañeda, 2018...). These multiscale schemes account for the abovementioned peculiarities of the mechanical behavior of the studied materials (strong and evolving 
plastic anisotropy and strength asymmetry), and hence offer good versatility to predict stress and strain anisotropy. Despite their undeniable advantages (physical foundations, accuracy, versatility...), the use of multiscale schemes is limited by the huge CPU time required by complex numerical applications, such as the prediction of the ductility limit, which is the primary concern of the current contribution. In fact, the CPU time required for the prediction of a complete FLD (i.e., strain paths ranging from uniaxial to equibiaxial tensile states), when using multiscale schemes for polycrystalline aggregates made of a few thousands of single crystals, ranges from days to weeks (Akpama et al., 2017; Gupta et al., 2018). To reduce the CPU time, it is more convenient to use phenomenological constitutive approaches instead of multiscale schemes in the modeling of the mechanical behavior of HCP polycrystalline materials. To this end, Cazacu and Barlat (2004) have developed an isotropic yield function to account for the asymmetry between tension and compression in the mechanical response. Some comparisons with results of polycrystalline simulations performed by Hosford and Allen (1973) reveal that the yield function proposed by Cazacu and Barlat (2004) describes very well the yielding asymmetry. The isotropic model developed in Cazacu and Barlat (2004) has been extended to plastic orthotropy in Cazacu et al. (2006). Known as CPB06, this extension takes into account the plastic orthotropy by introducing a linear transformation for the stress deviator tensor (through a transformation matrix). While it enables to reproduce with great accuracy the initial anisotropy of the studied materials, the CPB06 cannot accurately predict the evolving anisotropy due to the evolution of crystallographic texture. To better capture the evolution of plastic anisotropy and anisotropic hardening during loading, Plunkett et al. (2006) have developed an extension of the CPB06 model. In the latter investigation, the differential yield strengthening in compression and tension is described by predicting the components of the transformation matrix as well as strength differential parameters for different levels of plastic deformation. With this extension, a linear interpolation has been used to determine the evolution of these parameters. Another extension of the CPB06 model has been suggested by Plunkett et al. (2008). Instead of a single transformation, as introduced in CPB06, they used two independent linear transformations. It has been shown in Plunkett et al. (2008) that the accuracy in the description of the details of the plastic flow and anisotropy in both tension and compression can be further increased if more than two linear transformations were included in the formulation. The Cazacu models and their subsequent extensions have been widely used to model the mechanical behavior of various HCP materials and alloys. For instance, good correlations were observed between experimental results and numerical predictions obtained by Cazacu's models for the mechanical behavior of HCP materials, such as high-purity $\alpha$-titanium (Nixon et al., 2010), and titanium alloy Ti-6Al-4V (Gilles et al., 2011; Khan et al., 2012). The Cazacu anisotropic functions have also been used to model plastic anisotropy of magnesium alloys, such as AZ31 alloy (Muhammed et al., 2015; Soare et al., 2016), and ZEK100 alloy 
(Muhammed et al., 2015; Abedini et al., 2017). Despite the relevance of the Cazacu models in the description of plastic anisotropy and strength asymmetry of HCP materials, a number of investigators suggested using more elaborate plasticity models. Indeed, the coexistence of slip and twinning modes leads to complex physical mechanisms causing a significant change in the shape of the yield surface with accumulated plastic deformation, which cannot be described by traditional single yield surface. In elaborate plasticity models, plastic anisotropy resulting from slip and that induced by twinning modes are treated separately by two yield functions: a symmetric yield function to model the slip mode, and an asymmetric yield function representing twinning-dominated flow. The two-surface modeling concept has been introduced in Mróz (1967) and Krieg (1975). This concept has recently been applied in Lee et al. (2008) to model the mechanical behavior of HCP materials, especially AZ31 magnesium alloy sheets. In the latter model, each surface is defined by the Drucker-Prager yield function, suitably modified to account for the anisotropy of magnesium alloy sheets. The same concept has been used by Kim et al. (2013) to model temperature dependent anisotropic/asymmetric plastic behavior of AZ31 magnesium alloy sheets, where the Hill'48 (resp. CPB06) yield function has been used to model the slip (resp. twinning) dominant mode. More recently, Steglich et al. (2016) have used a twosurface approach to model the evolution of plastic anisotropy in rolled thin sheets made of two different rolled magnesium alloys (AZ31 and ZE10). In the latter contribution, the Barlat (Barlat, 1991) and the CPB06 yield functions have been employed to model plastic anisotropy due to slip and twinning modes, respectively. Following this study, Madi et al. (2017) have developed an identification strategy, which includes specimens along the plate principal directions and off-axes specimens along diagonal orientations in principal planes, and this strategy has been extended by Kondori et al. (2019) to model plastic anisotropy in thick plates (3D modeling). The constitutive framework developed in Steglich et al. (2016), Madi et al. (2017) and Kondori et al. (2019) is used in the current contribution after its extension to incorporate the effect of distortional hardening on the macroscopic behavior. In fact, it is well known that for HCP materials, the twinning activity is gradually exhausted and the asymmetry decreases or even reverses (Lou et al., 2007; Kondori and Benzerga, 2014). This behavior cannot be captured using constant anisotropy coefficients, as it is the case in the original model developed in Steglich et al. (2016), Madi et al. (2017) and Kondori et al. (2019). An attempt to remedy this limitation in the context of HCP sheet alloys has recently been proposed by Yoon et al. (2013), who considered that the anisotropy coefficients are dependent on the effective plastic strain. The approach proposed by Yoon et al. (2013) has been followed by other authors to accurately model the distortional hardening resulting from the effect of the evolution of the anisotropy parameters on the macroscopic mechanical response (Ghaffari Tari et al., 2014; Muhammad et al., 2015; Lee et al., 2017). This choice is motivated by the capabilities and versatility of this modeling approach, which have been proven by the comparison between 
experimental results and numerical predictions for a large variety of mechanical tests. The developed framework is based on a finite strain rate-independent formulation, with an associative flow rule and a plastic strain rate assumed to be normal to the activated yield functions. To numerically integrate the constitutive equations governing this model, we have developed an implicit integration scheme, which allows us to robustly switch from one active deformation mode to another. The accuracy and the efficiency of the developed numerical scheme are checked through several numerical results. The numerical predictions are assessed and validated by comparison with reference results taken from the literature (e.g., Steglich et al., 2016).

- Plastic instability prediction: during sheet metal forming processes, plastic deformation is often limited by the occurrence of plastic instability. To predict the onset of the latter and represent the corresponding necking limit strains in the form of FLDs, several plastic instability criteria have been developed in the literature. One of the most well-established and theoretically-sound criteria is the bifurcation approach initially proposed by Hill (1952). In the current contribution, the general bifurcation criterion (Drucker, 1950, 1956; Hill, 1958) has been used to predict the occurrence of diffuse necking in HCP thin sheets. This criterion will be called GBC in what follows. As to localized necking, it is predicted by another bifurcation criterion, based on the condition of loss of ellipticity of the governing equations (Rudnicki and Rice, 1975; Rice, 1976). This strain localization criterion, shortly denoted as RBC in what follows, is generally capable of predicting strain localization at realistic (finite) limit strains in the range of positive strain-path ratios when the constitutive framework exhibits some destabilizing effects, such as the development of vertices at the current points of the yield surface. Despite the development of vertex points when both slip and twinning modes are activated, it is shown in the present contribution that, within this particular two-surface plastic anisotropy constitutive framework, the Rice bifurcation criterion does not predict localized necking at realistic (finite) limit strains in the range of positive strain-path ratios. To overcome this limitation, the initial imperfection approach, developed by Marciniak and Kuczynski (1967) and briefly called hereafter $\mathrm{M}-\mathrm{K}$ approach, is adopted. For HCP materials, this approach has been coupled with a limited number of constitutive frameworks to predict the onset of plastic strain localization. For instance, Lévesque et al. (2016) have recently used a rate-dependent multiscale scheme based on the Taylor model to predict the occurrence of strain localization in magnesium alloys. With regard to phenomenological approaches, an extension of the CPB06 model, developed by Plunkett et al. (2006), has been coupled in Wu et al. (2015) with the M-K approach to predict forming limit diagrams for materials with HCP structure. However, the current contribution represents the first time that bifurcation criteria are applied for the prediction of diffuse and localized necking in HCP materials. The application of these criteria requires the analytical expression of the continuum tangent modulus to be determined. The 
derivation of such an elastoplastic tangent modulus is detailed in the present contribution. The coupling of the initial imperfection approach with the elastoplastic constitutive framework may be mathematically formulated as a strongly non-linear twenty-equation system (with twenty unknowns). We have developed an implicit algorithm to solve this set of equations. The adopted instability criteria are implemented using the multi-paradigm numerical computing environment Mathematica. Note that the developed prediction approaches are sufficiently general to be applied for the determination of necking in a wide range of HCP alloys. In the current investigation, these approaches are used to predict the formability limit of thin sheets made of magnesium alloy AZ31. This choice is motivated by the growing use of this well-known alloy in several industrial applications (automotive and aircraft industries) and by the availability of identified material parameters for this alloy in the literature (Steglich et al., 2016; Kondori et al., 2019). A sensitivity study is also conducted to analyze the effect of some material parameters on the predicted limit strains. Furthermore, the effect of distortional hardening on the onset of plastic instability is investigated.

A brief outline of the present paper is as follows:

- Section 2 details the constitutive framework selected to model the mechanical behavior of HCP materials as well as the main equations governing the adopted necking criteria.

- Section 3 outlines the numerical implementation of the constitutive equations coupled with the instability criteria presented in Section 2.

- Section 4 addresses the validation of the developed model and presents a numerical investigation to analyze the sensitivity of the predicted ductility limits to various material parameters.

- Section 5 closes our contribution by some conclusions and future work.

\section{Theoretical framework}

In this section, the main equations that describe the two-surface constitutive framework are presented. Then, the expression of the analytical tangent modulus is derived from these constitutive equations. This elastoplastic tangent modulus is required in the mathematical formulation of the necking criteria presented at the end of this section.

\subsection{Constitutive equations}

The constitutive modeling adopted in the current investigation is taken to be rate-independent and is formulated in the finite strain range. As a departure point of the theoretical framework developed in the subsequent sections, the spatial velocity gradient $\mathbf{g}$ is additively decomposed into its symmetric and skew-symmetric part, denoted $\mathbf{d}$ and $\mathbf{w}$, respectively: 


$$
\mathbf{g}=\mathbf{d}+\mathbf{w}
$$

To satisfy the objectivity principle (i.e., frame invariance), objective derivatives for tensor variables should be used (Sidoroff and Dogui, 2001). A practical approach, adopted to ensure frame invariance while maintaining simple forms for the constitutive equations, consists in reformulating these equations in terms of rotation-compensated variables. In the present work, a co-rotational approach based on the Jaumann objective rate is used. Hence, tensor quantities are expressed in a rotating frame, so that simple material time derivatives can be used in the constitutive equations. The rotation $\mathbf{r}$, which defines this rotating frame with respect to the fixed one, is derived from the spin tensor $\mathbf{w}$ (skew-symmetric part of $\mathbf{g}$ ) through the following relation:

$$
\dot{\mathbf{r}} \cdot \mathbf{r}^{T}=\mathbf{w}
$$

with $\mathbf{r}^{T}$ is the transpose of tensor $\mathbf{r}$.

In the remainder of Section 2.1, all tensor variables will be expressed in the rotating frame (called corotational frame), that is to say, using rotation-compensated variables. Consequently, time derivatives are involved in the constitutive equations, making them identical in form to a small-strain formulation. The strain rate $\mathbf{d}$ is itself split into its elastic part $\mathbf{d}^{e}$ and plastic part $\mathbf{d}^{p}$ :

$$
\mathbf{d}=\mathbf{d}^{e}+\mathbf{d}^{p} .
$$

The stress rate is described with a hypoelastic law:

$$
\dot{\sigma}=\mathbf{C}^{e}: \mathbf{d}^{e},
$$

where $\mathbf{C}^{e}$ denotes the fourth-order elasticity tensor. Here, elasticity is assumed to be isotropic and is defined by two material parameters: the Young modulus $E$ and the Poisson ratio $v$.

The plastic strain rate $\mathbf{d}^{p}$ is assumed to result from a contribution due to slip-dominated deformation mode, denoted $\mathbf{d}^{p s}$, and a contribution induced by twinning-affected deformation mode, designated as $\mathbf{d}^{\text {pt }}$ (Steglich et al., 2016; Kondori et al., 2019):

$$
\mathbf{d}^{p}=\mathbf{d}^{p s}+\mathbf{d}^{p t} .
$$

Both $\mathbf{d}^{p s}$ and $\mathbf{d}^{p t}$ are given by an associative plastic flow rule (normality law):

$$
\mathbf{d}^{p s}=\dot{\gamma}^{s} \frac{\partial \Phi^{s}}{\partial \boldsymbol{\sigma}}, \quad \mathbf{d}^{p t}=\dot{\gamma}^{t} \frac{\partial \Phi^{t}}{\partial \boldsymbol{\sigma}}
$$

where:

- $\Phi^{s}$ (resp. $\Phi^{t}$ ) represents the slip (resp. twinning) yield function and is equal to $\widehat{\sigma}^{s}-R^{s}$ (resp. $\left.\hat{\sigma}^{t}-R^{t}\right)$

- $\dot{\gamma}^{s}$ (resp. $\dot{\gamma}^{t}$ ) is the equivalent slip (resp. twinning) strain rate (also called the plastic multiplier),

- $\hat{\sigma}^{s}$ (resp. $\hat{\sigma}^{t}$ ) stands for the equivalent slip (resp. twinning) stress, 
- $R^{s}$ (resp. $R^{t}$ ) denotes the slip (resp. twinning) yield stress. In the current contribution, both self-hardening (i.e., the effect of the plastic strain of a deformation mode to increase the yield stress of the same mode) and latent hardening (i.e., the effect of the plastic strain of a deformation mode to increase the yield stress of the other mode) are considered. Hence, $R^{s}$ and $R^{t}$ are defined by the following generic forms:

$$
R^{s} \triangleq R^{s}\left(\gamma^{s}, \gamma^{t}\right), \quad R^{t} \triangleq R^{t}\left(\gamma^{s}, \gamma^{t}\right)
$$

It has been shown in several previous contributions that slip-induced plasticity is almost insensitive to the stress sign (i.e., the tensile and compressive stresses required for the activation of the slip mode are equal in absolute value). Furthermore, the small number of slip systems in HCP materials leads to a strong evolution of crystallographic texture, and hence, of plastic anisotropy. According to Steglich et al. (2016) and Kondori et al. (2019), the specific characteristics of tension/compression asymmetry and strong evolution of plastic anisotropy are accurately accounted for by the anisotropic yield function developed by Barlat et al. (1991) and briefly called BLB91. In the BLB91 model, the equivalent slip stress $\hat{\sigma}^{s}$ is defined by the following expression:

$$
\hat{\sigma}^{s}=\left(\frac{1}{2}\left(\left|\Sigma_{1}^{s}-\Sigma_{2}^{s}\right|^{a^{s}}+\left|\Sigma_{2}^{s}-\Sigma_{3}^{s}\right|^{a^{s}}+\left|\Sigma_{1}^{s}-\Sigma_{3}^{s}\right|^{a^{s}}\right)\right)^{1 / a^{s}} .
$$

In the above equation, $\Sigma_{1}^{s}, \Sigma_{2}^{s}$ and $\Sigma_{3}^{s}$ are the eigenvalues of a tensor $\Sigma^{s}\left(\Sigma_{1}^{s} \geq \Sigma_{2}^{s} \geq \Sigma_{3}^{s}\right)$, which is obtained from the Cauchy stress tensor $\sigma$ by the following linear transformation:

$$
\Sigma^{s}=\left(\mathbf{L}^{s} . \mathbf{T}\right): \boldsymbol{\sigma}
$$

where $\mathbf{L}^{s}$ is a fourth-order transformation tensor defined in a matrix form as follows:

$$
\mathbf{L}^{s}=\left(\begin{array}{cccccc}
l_{11}^{s} & l_{12}^{s} & l_{13}^{s} & 0 & 0 & 0 \\
l_{12}^{s} & l_{22}^{s} & l_{23}^{s} & 0 & 0 & 0 \\
l_{13}^{s} & l_{23}^{s} & l_{33}^{s} & 0 & 0 & 0 \\
0 & 0 & 0 & l_{44}^{s} & 0 & 0 \\
0 & 0 & 0 & 0 & l_{55}^{s} & 0 \\
0 & 0 & 0 & 0 & 0 & l_{66}^{s}
\end{array}\right),
$$

and matrix $\mathbf{T}$ is given by:

$$
\mathbf{T}=\frac{1}{3}\left(\begin{array}{cccccc}
2 & -1 & -1 & 0 & 0 & 0 \\
-1 & 2 & -1 & 0 & 0 & 0 \\
-1 & -1 & 2 & 0 & 0 & 0 \\
0 & 0 & 0 & 3 & 0 & 0 \\
0 & 0 & 0 & 0 & 3 & 0 \\
0 & 0 & 0 & 0 & 0 & 3
\end{array}\right)
$$


The plastic deformation behavior of HCP materials is characterized by the yielding asymmetry of the stress state between tension and compression. This asymmetry is due to the activation of the twining deformation mode. To accurately capture the effect of this asymmetry on the shape of the anisotropic yield function, we have used the CPB06 yield criterion (Cazacu et al., 2006) to compute the equivalent twinning stress $\hat{\sigma}^{t}$, which is defined by the following expression:

$$
\widehat{\sigma}^{t}=\left(\left(\left|\Sigma_{1}^{t}\right|-k \Sigma_{1}^{t}\right)^{a^{t}}+\left(\left|\Sigma_{2}^{t}\right|-k \Sigma_{2}^{t}\right)^{a^{t}}+\left(\left|\Sigma_{3}^{t}\right|-k \Sigma_{3}^{t}\right)^{a^{t}}\right)^{1 / a^{t}},
$$

where $k$ is a material parameter that describes the asymmetry degree of the deformation behavior, while $\Sigma_{1}^{t}, \Sigma_{2}^{t}$ and $\Sigma_{3}^{t}$ (with $\Sigma_{1}^{t} \geq \Sigma_{2}^{t} \geq \Sigma_{3}^{t}$ ) are the eigenvalues of a tensor $\Sigma^{t}$ related to $\sigma$ by:

$$
\Sigma^{t}=\left(\mathbf{L}^{t} \cdot \mathbf{T}\right): \sigma
$$

The orthotropic transformation matrix $\mathbf{L}^{t}$ is defined by the following expression:

$$
\mathbf{L}^{t}=\left(\begin{array}{cccccc}
l_{11}^{t} & l_{12}^{t} & l_{13}^{t} & 0 & 0 & 0 \\
l_{12}^{t} & l_{22}^{t} & l_{23}^{t} & 0 & 0 & 0 \\
l_{13}^{t} & l_{23}^{t} & l_{33}^{t} & 0 & 0 & 0 \\
0 & 0 & 0 & l_{44}^{t} & 0 & 0 \\
0 & 0 & 0 & 0 & l_{55}^{t} & 0 \\
0 & 0 & 0 & 0 & 0 & l_{66}^{t}
\end{array}\right) .
$$

The material parameters $\left(a^{\circ}, k, l_{\circ}^{\circ}\right)$ involved in Eqs. (8), (10), (12) and (14) are classically identified on the basis of a set of mechanical tests (uniaxial tension, uniaxial compression, simple shear...). An experimental methodology developed to identify these parameters has been presented and extensively discussed in Steglich et al. (2016) and Kondori et al. (2019).

The plastic flow may occur by activating one of the two plastic deformation modes (slip or twinning) or both modes simultaneously. The activation of each deformation mode is governed by the following Kuhn-Tucker constraints:

$$
\begin{aligned}
& \text { slip mode: } \quad \Phi^{s}=\left(\widehat{\sigma}^{s}-R^{s}\right) \leq 0, \quad \dot{\gamma}^{s} \geq 0, \quad \Phi^{s} \dot{\gamma}^{s}=0, \\
& \text { twinning mode: } \Phi^{t}=\left(\hat{\sigma}^{t}-R^{t}\right) \leq 0, \quad \dot{\gamma}^{t} \geq 0, \quad \Phi^{t} \dot{\gamma}^{t}=0 \text {. }
\end{aligned}
$$

The possible scenarios for the activation of the different deformation modes are illustrated in Fig. 1, where the distinct yield surfaces are plotted in the $\sigma_{11}-\sigma_{22}$ space normalized by the initial slip yield stress $\sigma_{0}$. 


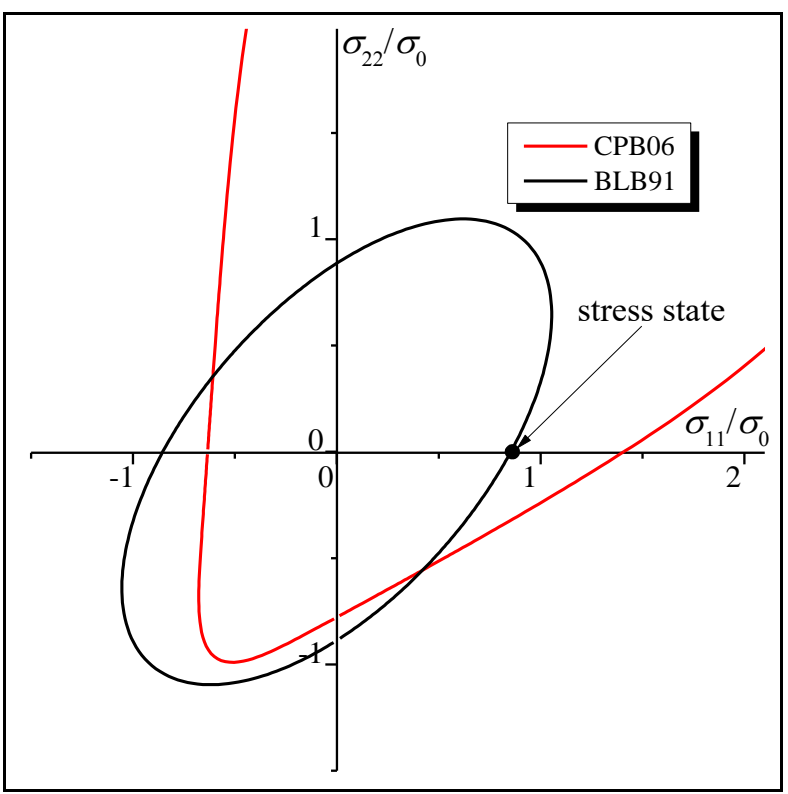

(a)

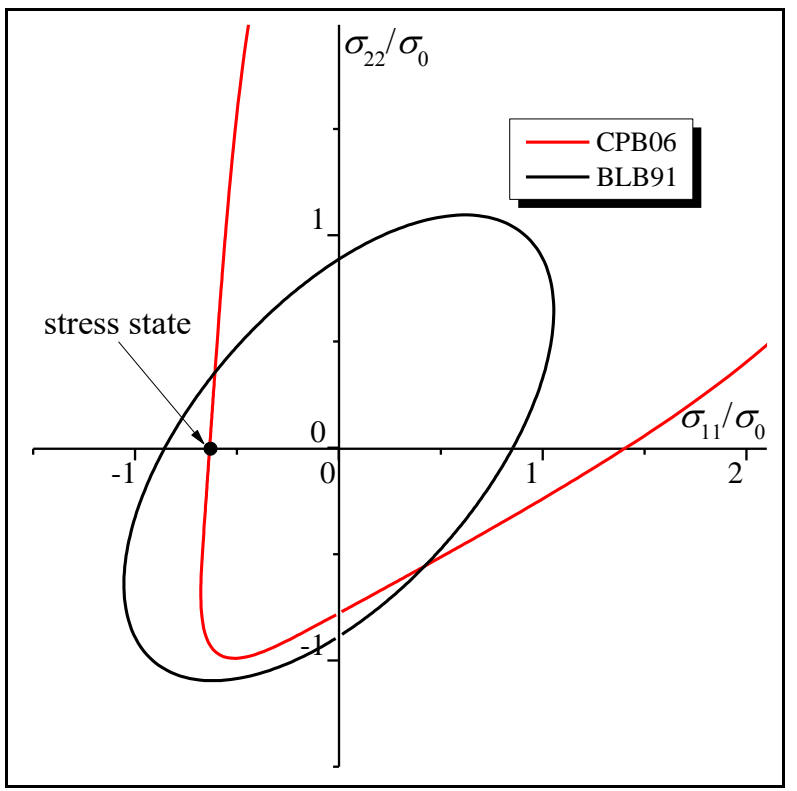

(b)

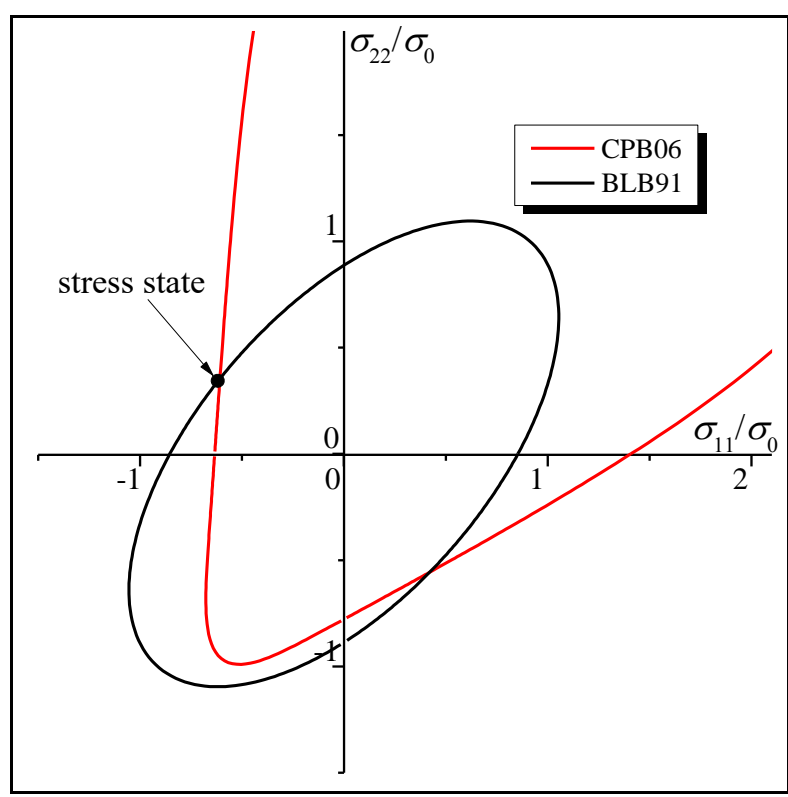

(c)

Fig. 1. Possible scenarios for the activation of the different deformation modes: (a) Slip mode only; (b) Twinning mode only; (c) Slip and twinning modes simultaneously.

Note that if the anisotropy transformation matrices $\mathbf{L}^{s}$ and $\mathbf{L}^{t}$ and the strength differential parameter $k$ are taken to be constant, Eqs. (15) describe isotropic hardening, i.e. a proportional expansion of the two yield surfaces, without any shape change. To accurately account for the effect of the rapid change of plastic anisotropy in the modeling of the mechanical behavior, we assume that the different anisotropy parameters (i.e., the components of matrices $\mathbf{L}^{s}$ and $\mathbf{L}^{t}$ and parameter $k$ ) are dependent on the equivalent slip and twinning strains $\gamma^{s}$ and $\gamma^{t}$ :

$$
\mathbf{L}^{s} \triangleq \mathbf{L}^{s}\left(\gamma^{s}, \gamma^{t}\right), \quad \mathbf{L}^{t} \triangleq \mathbf{L}^{t}\left(\gamma^{s}, \gamma^{t}\right), \quad k \triangleq k\left(\gamma^{s}, \gamma^{t}\right)
$$


The consideration of the evolution of the anisotropy parameters during plastic loading allows incorporating the effect of distortional hardening, in addition to isotropic hardening, on the macroscopic response (Yoon et al., 2013; Ghaffari Tari et al., 2014; Muhammad et al., 2015; Lee et al., 2017).

The Kuhn-Tucker constraints given by Eq. (15) (two inequalities and one equality for each deformation mode) can be equivalently expressed as a single equality by using the Fischer-Burmeister equality (Fischer, 1992; Fischer, 1997):

$$
\begin{array}{ll}
\text { slip mode: } & \Psi^{s}=\sqrt{\left(\Phi^{s}\right)^{2}+\left(\dot{\gamma}^{s}\right)^{2}}+\left(\Phi^{s}-\dot{\gamma}^{s}\right)=0, \\
\text { twinning mode: } & \Psi^{t}=\sqrt{\left(\Phi^{t}\right)^{2}+\left(\dot{\gamma}^{t}\right)^{2}}+\left(\Phi^{t}-\dot{\gamma}^{t}\right)=0 .
\end{array}
$$

The Fischer-Burmeister form is more convenient for the numerical implementation of the consistency condition. For instance, the Fischer-Burmeister form has been used in Akpama et al. (2016), instead of the classical formulation given by Eq. (15), in order to compute the slip rates within a rateindependent crystal plasticity constitutive framework. Adopting this compact form allows avoiding the recourse to the traditional elastic prediction-plastic correction algorithm, generally used to integrate rate-independent constitutive equations. Indeed, the use of the conventional form of the Kuhn-Tucker constraints may lead to some numerical difficulties when several deformation modes (more than one) are simultaneously or separately activated. These difficulties are related to the robust switch from one deformation mode to another. Hence, in the current work, Eq. (17) has been used to build the numerical scheme for the integration of the constitutive equations.

The specific expressions for the evolution laws of the components of the anisotropy parameters (i.e., the components of matrices $\mathbf{L}^{s}$ and $\mathbf{L}^{t}$ and parameter $k$ ) as well as the hardening yield functions $R^{s}$ and $R^{t}$ will be provided in Section 4 .

\subsection{Analytic computation of the continuum tangent modulus}

The constitutive framework described in Section 2.1 is complemented here by the derivation of the expression of the analytical tangent modulus $\mathcal{L}$, which is essential for the subsequent developments related to the analysis of diffuse and localized necking. This tangent modulus relates the nominal stress rate $\dot{\mathbf{n}}$ to the velocity gradient $\mathbf{g}$, as expressed by the following relation:

$$
\dot{\mathbf{n}}=\mathcal{L}: \mathbf{g}
$$

To derive the expression of $\mathcal{L}$, let us first recall the relationship between the Cauchy stress tensor $\sigma$ and the nominal stress tensor $\mathbf{n}$ :

$$
\mathbf{n}=j \mathbf{f}^{-1} \cdot \boldsymbol{\sigma},
$$

where $\mathbf{f}$ is the deformation gradient and $j$ its determinant. By taking the time derivative of Eq. (19), one obtains: 


$$
\dot{\mathbf{n}}=j \mathbf{f}^{-1} \cdot(\dot{\sigma}+\sigma \operatorname{Tr}(\mathbf{d})-\mathbf{g} \cdot \boldsymbol{\sigma}) .
$$

In the current investigation, an updated Lagrangian approach is adopted. Accordingly, $\mathbf{f}$ is set to the second-order identity tensor $\mathbf{I}_{2}$ and $j$ is set to 1. Hence, Eq. (20) reduces to:

$$
\dot{\mathbf{n}}=\dot{\boldsymbol{\sigma}}+\boldsymbol{\sigma} \operatorname{Tr}(\mathbf{d})-\mathbf{g} . \boldsymbol{\sigma} .
$$

Using Eq. (1) and the Jaumann derivative of the Cauchy stress tensor $\sigma$, Eq (21) can be rewritten in the following form after some straightforward developments (Haddag et al., 2009):

$$
\dot{\mathbf{n}}=\mathbf{C}^{e}: \mathbf{d}^{e}+\sigma \operatorname{Tr}(\mathbf{d})-\mathbf{d} . \sigma-\sigma . \mathbf{w}
$$

To derive a constitutive relation expressed in the form of Eq. (18), each of the terms on the right-hand side of Eq. (22) is conveniently rewritten so as to obtain the following relations:

$$
\left\{\begin{array} { l } 
{ \mathbf { C } ^ { e } : \mathbf { d } ^ { e } = \mathbf { C } ^ { e p } : \mathbf { g } } \\
{ \boldsymbol { \sigma } \operatorname { T r } ( \mathbf { d } ) = \mathbf { C } _ { 1 } : \mathbf { g } } \\
{ \mathbf { d } . \boldsymbol { \sigma } = \mathbf { C } _ { 2 } : \mathbf { g } } \\
{ \boldsymbol { \sigma } . \mathbf { w } = \mathbf { C } _ { 3 } : \mathbf { g } }
\end{array} \quad , \quad \text { with } \left\{\begin{array}{l}
C_{1 i j k l}=\sigma_{i j} \delta_{k l} \\
C_{2 i j k l}=\frac{1}{2}\left(\sigma_{j l} \delta_{i k}+\sigma_{j k} \delta_{i l}\right), \\
C_{3 i j k l}=\frac{1}{2}\left(\sigma_{i k} \delta_{j l}-\sigma_{i l} \delta_{j k}\right)
\end{array}\right.\right.
$$

where $\delta_{k l}$ is the Kronecker delta.

The analytical tangent modulus $\mathcal{L}$ is finally derived by combining Eqs. (22) and (23):

$$
\mathcal{L}=\mathbf{C}^{e p}+\mathbf{C}_{1}-\mathbf{C}_{2}-\mathbf{C}_{3} .
$$

In what follows, we provide in some details the computation of the elastoplastic tangent modulus $\mathbf{C}^{e p}$. In the subsequent developments, we assume that the two deformation modes (namely slip and twining) are simultaneously activated. The special case when only one deformation mode is activated is addressed at the end of this section.

The elastoplastic tangent modulus $\mathbf{C}^{e p}$ is defined as follows:

$$
\dot{\sigma}=\mathbf{C}^{e}: \mathbf{d}^{e}=\mathbf{C}^{e}:\left(\mathbf{d}-\mathbf{d}^{p s}-\mathbf{d}^{p t}\right)=\mathbf{C}^{e p}: \mathbf{d}=\mathbf{C}^{e p}: \mathbf{g} .
$$

As the two deformation modes are activated, the Kuhn-Tucker conditions given by Eq. (15) are written in their rate forms:

$$
\begin{array}{ll}
\text { slip mode: } & \dot{\Phi}^{s}=\mathbf{V}_{\sigma}^{s}: \dot{\sigma}+M^{s s} \dot{\gamma}^{s}+M^{s t} \dot{\gamma}^{t}=0, \\
\text { twinning mode: } & \dot{\Phi}^{t}=\mathbf{V}_{\sigma}^{t}: \dot{\sigma}+M^{t s} \dot{\gamma}^{s}+M^{t t} \dot{\gamma}^{t}=0,
\end{array}
$$

where:

$$
\begin{aligned}
& \mathbf{V}_{\sigma}^{s}=\frac{\partial \hat{\sigma}^{s}}{\partial \boldsymbol{\sigma}}, \quad M^{s s}=\frac{\partial \Phi^{s}}{\partial \gamma^{s}}, \quad M^{s t}=\frac{\partial \Phi^{s}}{\partial \gamma^{t}} \\
& \mathbf{V}_{\sigma}^{t}=\frac{\partial \hat{\sigma}^{t}}{\partial \boldsymbol{\sigma}}, \quad M^{t s}=\frac{\partial \Phi^{t}}{\partial \gamma^{s}}, \quad M^{t t}=\frac{\partial \Phi^{t}}{\partial \gamma^{t}}
\end{aligned}
$$


The expressions of $\partial \widehat{\sigma}^{s} / \partial \sigma, \partial \hat{\sigma}^{t} / \partial \sigma, M^{s s}, M^{s t}, M^{t s}$ and $M^{t t}$ are given in Appendix A.

By combining Eqs. (3)-(6), one can obtain the following expression for the stress rate $\dot{\sigma}$ :

$$
\dot{\sigma}=\mathbf{C}^{e}:\left(\mathbf{d}-\dot{\gamma}^{s} \mathbf{V}_{\sigma}^{s}-\dot{\gamma}^{t} \mathbf{V}_{\sigma}^{t}\right) \text {. }
$$

Using Eqs. (27) and (28), Eq. (26) can be reformulated as:

$$
\forall \alpha=s, t: \quad \dot{\Phi}^{\alpha}=\mathbf{V}_{\sigma}^{\alpha}: \mathbf{C}^{e}:\left(\mathbf{d}-\dot{\gamma}^{\beta} \mathbf{V}_{\sigma}^{\beta}\right)+M^{\alpha \beta} \dot{\gamma}^{\beta}=0, \quad \beta=s, t,
$$

which can be expressed in a more compact matrix form:

$$
\mathbf{A} \cdot \dot{\gamma}=\mathbf{Y}
$$

where:

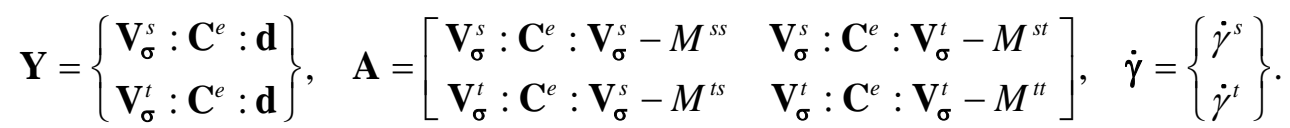

Hence, the components of vector $\dot{\gamma}$ can be determined by solving Eq. (30):

$$
\forall \alpha=s, t: \quad \dot{\gamma}^{\alpha}=\left[B^{\alpha \beta} \mathbf{V}_{\sigma}^{\beta}: \mathbf{C}^{e}\right]: \mathbf{d}, \quad \beta=s, t,
$$

where $\mathbf{B}$ is the inverse of matrix $\mathbf{A}$.

Substituting vector $\dot{\gamma}$ into Eq. (28) allows the following expression for $\dot{\sigma}$ to be obtained:

$$
\dot{\sigma}=\left(\mathbf{C}^{e}-\left(\mathbf{C}^{e}: \mathbf{V}_{\sigma}^{\alpha}\right) \otimes \mathbf{X}^{\alpha}\right): \mathbf{d}, \quad \alpha=s, t \quad \text { with } \quad \mathbf{X}^{\alpha}=B^{\alpha \beta} \mathbf{V}_{\sigma}^{\beta}: \mathbf{C}^{e}, \quad \beta=s, t .
$$

The comparison between Eq. (25) and Eq. (33) allows identifying the expression of the elastoplastic tangent modulus $\mathbf{C}^{e p}$ :

$$
\mathbf{C}^{e p}=\mathbf{C}^{e}-\left(\mathbf{C}^{e}: \mathbf{V}_{\boldsymbol{\sigma}}^{\alpha}\right) \otimes \mathbf{X}^{\alpha}, \quad \alpha=s, t .
$$

When the mechanical behavior is purely elastic (neither of the deformation modes is activated), Eq. (34) reduces to:

$$
\mathbf{C}^{e p}=\mathbf{C}^{e} .
$$

In case where only one deformation mode is activated, Eq. (34) reduces to the following expression:

$$
\begin{array}{ll}
\text { slip mode : } & \mathbf{C}^{e p}=\mathbf{C}^{e}-\frac{\left(\mathbf{C}^{e}: \mathbf{V}_{\sigma}^{s}\right) \otimes\left(\mathbf{V}_{\sigma}^{s}: \mathbf{C}^{e}\right)}{\mathbf{V}_{\sigma}^{s}: \mathbf{C}^{e}: \mathbf{V}_{\sigma}^{s}-M^{s s}}, \\
\text { twinning mode }: & \mathbf{C}^{e p}=\mathbf{C}^{e}-\frac{\left(\mathbf{C}^{e}: \mathbf{V}_{\sigma}^{t}\right) \otimes\left(\mathbf{V}_{\sigma}^{t}: \mathbf{C}^{e}\right)}{\mathbf{V}_{\sigma}^{t}: \mathbf{C}^{e}: \mathbf{V}_{\sigma}^{t}-M^{t t}}
\end{array}
$$

Once determined, the expression of the elastoplastic tangent modulus $\mathbf{C}^{e p}$ is introduced in Eq. (24) to compute the analytical tangent modulus $\mathcal{L}$. 


\subsection{Necking criteria}

\subsubsection{General plane-stress state}

An orthogonal Cartesian coordinate system $\left(\overrightarrow{\mathbf{x}}_{1}, \overrightarrow{\mathbf{x}}_{2}, \overrightarrow{\mathbf{x}}_{3}\right)$ is defined, where axes $\overrightarrow{\mathbf{x}}_{1}, \overrightarrow{\mathbf{x}}_{2}$ and $\overrightarrow{\mathbf{x}}_{3}$ coincide with the rolling (L), transverse (T), and normal (S) directions of the sheet, respectively. To predict the necking limit strains, we consider a quasi-static deformation of a thin HCP sheet submitted to in-plane biaxial loading in the plane $\left(\overrightarrow{\mathbf{x}}_{1}, \overrightarrow{\mathbf{x}}_{2}\right)$. Considering the small thickness of the sheet, and following most studies on sheet metal formability, a generalized plane-stress state is assumed (Hutchinson et al., 1978a). With this assumption, the out-of-plane components of the Cauchy stress tensor are equal to zero, namely:

$$
\boldsymbol{\sigma} \cdot \overrightarrow{\mathbf{x}}_{3}=\overrightarrow{0} \Leftrightarrow \sigma_{13}=\sigma_{23}=\sigma_{33}=0 .
$$

The metal sheet is taken to be initially stress-free. Hence, the plane-stress conditions can be expressed in terms of the components of the nominal stress rate:

$$
\dot{n}_{13}=\dot{n}_{31}=\dot{n}_{23}=\dot{n}_{32}=\dot{n}_{33}=0 \text {. }
$$

Eq. (38) also implies that the out-of-plane components of the velocity gradient $\mathbf{g}$ are equal to zero:

$$
g_{13}=g_{31}=g_{23}=g_{32}=0 .
$$

The general constitutive equation (18) can be expressed under the plane-stress conditions as follows (Akpama et al., 2017):

$$
\dot{\tilde{\mathbf{n}}}=\tilde{\mathcal{L}}: \tilde{\mathbf{g}},
$$

where:

$$
\begin{aligned}
& \dot{\tilde{\mathbf{n}}}=\left[\begin{array}{ll}
\dot{n}_{11} & \dot{n}_{12} \\
\dot{n}_{21} & \dot{n}_{22}
\end{array}\right], \quad \tilde{\mathbf{g}}=\left[\begin{array}{ll}
g_{11} & g_{12} \\
g_{21} & g_{22}
\end{array}\right], \\
& \forall \alpha, \beta, \gamma, \delta=1,2: \quad \tilde{\mathcal{L}}_{\alpha \beta \gamma \delta}=\mathcal{L}_{\alpha \beta \gamma \delta}-\frac{\mathcal{L}_{\alpha \beta 33} \mathcal{L}_{33 \gamma \delta}}{\mathcal{L}_{3333}} .
\end{aligned}
$$

\subsubsection{Bifurcation criteria}

Diffuse necking is predicted here by the general bifurcation criterion (GBC). This criterion states that plastic instability occurs when the second-order work vanishes (Drucker, 1950, 1956; Hill, 1958). To mathematically formulate this criterion, let us introduce the tangent modulus $\tilde{\mathcal{L}}^{\text {p }}$ relating the rate of the in-plane first Piola-Kirchhoff stress $\tilde{\mathbf{p}}$ to the rate of the in-plane deformation gradient $\tilde{\mathbf{f}}$ :

$$
\dot{\tilde{\mathbf{p}}}=\tilde{\mathcal{L}}^{\mathrm{p}}: \dot{\tilde{\mathbf{f}}}
$$

The tangent modulus $\tilde{\mathcal{L}}^{\text {p }}$ is obtained from the analytical tangent modulus $\tilde{\mathcal{L}}$ introduced in Eq. (40) by permutation of the first two indices (Bouktir et al., 2018): 


$$
\forall i, j, k, l=1,2: \quad \tilde{\mathcal{L}}_{i j k l}^{p}=\tilde{\mathcal{L}}_{j k l} .
$$

According to the GBC, diffuse necking is predicted when at least one eigenvalue of the symmetric part of $\tilde{\mathcal{L}}^{\text {p }}$ (called hereafter $\tilde{\mathcal{L}}_{\text {sym }}^{\text {p }}$ ) becomes negative (Bouktir et al., 2018).

The bifurcation theory has also been used to derive a localized necking criterion. This material instability approach, initially developed by Rudnicki and Rice (1975) and Rice (1976), states that plastic strain localization occurs when the determinant of the acoustic tensor vanishes. This Rice bifurcation criterion $(\mathrm{RBC})$ is mathematically expressed by the following equation:

$$
\operatorname{det}(\overrightarrow{\mathbf{v}} \cdot \tilde{\mathcal{L}} \cdot \overrightarrow{\mathbf{v}})=0
$$

where $\overrightarrow{\mathbf{v}}$ is the unit vector normal to the localization band and lying in the plane $\left(\overrightarrow{\mathbf{x}}_{1}, \overrightarrow{\mathbf{x}}_{2}\right)$. In the frame $\left(\overrightarrow{\mathbf{x}}_{1}, \overrightarrow{\mathbf{x}}_{2}\right)$, vector $\overrightarrow{\mathbf{v}}$ is given by the following components $(\cos \theta, \sin \theta)$ (with $\mathrm{O}^{\circ} \leq \boldsymbol{\theta} \leq 90^{\circ}$ ). Further details about the Rice bifurcation approach can be found in Ben Bettaieb and Abed-Meraim (2015).

\subsubsection{Initial imperfection approach}

The occurrence of localized necking is also predicted by using the imperfection approach, initially proposed by Marciniak and Kuczynski (1967). The initial version of this approach has been restricted to the prediction of the formability limits in the range of positive strain-path ratios (between the planestrain tension state and the equibiaxial tension state). Its extension to the range of negative strain-path ratios has been developed by Hutchinson et al. (1978b). Hereafter, the extended form of the initial imperfection approach ( $\mathrm{M}-\mathrm{K}$ theory) will be used. The $\mathrm{M}-\mathrm{K}$ analysis is based on the assumption of the preexistence of an initial geometric imperfection, in the form of a narrow band across the thickness of the metal sheet, as shown in Fig. 2.

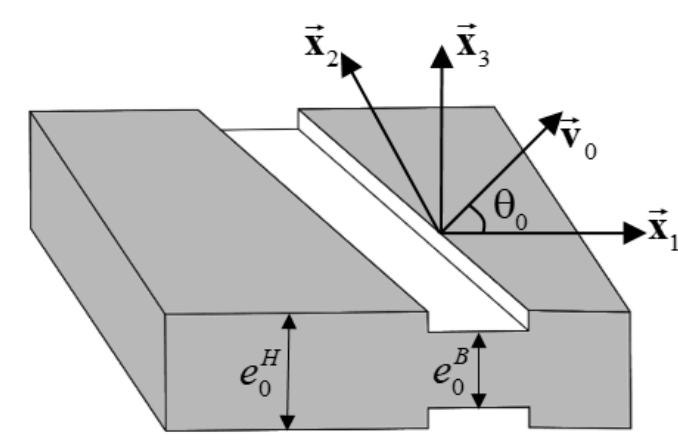

(a)

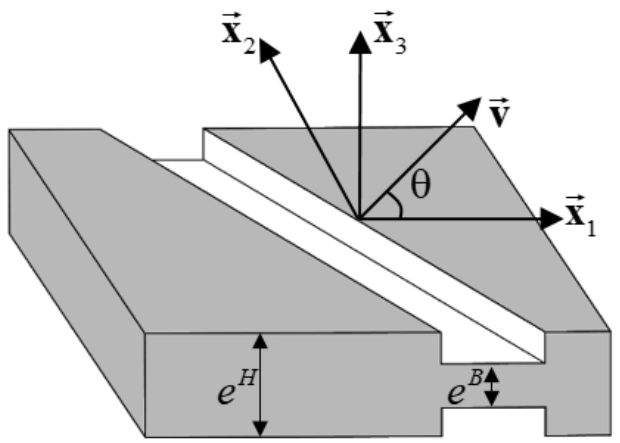

(b)

Fig. 2. Initial imperfection approach: (a) Configuration before loading; (b) Configuration after loading.

The $\mathrm{M}-\mathrm{K}$ analysis is governed by three main equations: 
- The kinematic compatibility condition between the band zone (designated as ' $B$ ') and the homogeneous zone (designated as ' $H$ '). This condition allows linking the in-plane velocity gradient in the band zone $\tilde{\mathbf{g}}^{B}$ to its counterpart in the homogeneous zone $\tilde{\mathbf{g}}^{H}$, through a jump vector $\dot{\overrightarrow{\mathbf{c}}}$ and vector $\overrightarrow{\mathbf{v}}$ normal to the band:

$$
\tilde{\mathbf{g}}^{B}=\tilde{\mathbf{g}}^{H}+\dot{\overrightarrow{\mathbf{c}}} \otimes \overrightarrow{\mathbf{v}}
$$

- The force equilibrium across the interface between the band and the homogeneous zone:

$$
\xi \overrightarrow{\mathbf{v}} . \dot{\tilde{\mathbf{n}}}^{B}=\overrightarrow{\mathbf{v}} \cdot \dot{\tilde{\mathbf{n}}}^{H}
$$

where $\xi$ is the relative thickness of the band zone, which is equal to the ratio $\mathrm{e}^{B} / \mathrm{e}^{H}$. Here $\mathrm{e}^{B}$ (resp. $\mathrm{e}^{H}$ ) is the current thickness of the band zone (resp. the homogeneous zone).

- The plane-stress constitutive framework both in the band and in the homogeneous zone:

$$
\dot{\tilde{\mathbf{n}}}^{B}=\tilde{\mathcal{L}}^{B}: \tilde{\mathbf{g}}^{B} \quad ; \quad \dot{\tilde{\mathbf{n}}}^{H}=\tilde{\mathcal{L}}^{H}: \tilde{\mathbf{g}}^{H} .
$$

The combination of equations (45), (46) and (47)) allows the following expression for the jump vector $\dot{\overrightarrow{\mathbf{c}}}$ to be obtained:

$$
\dot{\overrightarrow{\mathbf{c}}}=\left(\overrightarrow{\mathbf{v}} \cdot \tilde{\mathcal{L}}^{B} \cdot \overrightarrow{\mathbf{v}}\right)^{-1}\left(\overrightarrow{\mathbf{v}} \cdot\left(\tilde{\mathcal{L}}^{H}-\xi \tilde{\mathcal{L}}^{B}\right): \tilde{\mathbf{g}}^{H}\right)
$$

Equations (45)-(47) are supplemented by the following two evolution equations:

- The evolution of the relative thickness of the band zone $\xi$ as a function of its initial value $\xi_{0}$ and the strain components normal to the plane of the sheet $\varepsilon_{33}^{B}$ and $\varepsilon_{33}^{H}$ :

$$
\xi=\xi_{0} e^{\left(\varepsilon_{33}^{B}-\varepsilon_{33}^{H}\right)} .
$$

- The evolution of the band orientation, defined by vector $\overrightarrow{\mathbf{v}}=(\cos (\theta), \sin (\theta))$, as a function of its initial value $\theta_{0}$ and the strain components $\varepsilon_{11}^{H}$ and $\varepsilon_{22}^{H}$ :

$$
\theta=\arctan \left[\tan \left(\theta_{0}\right) e^{\left(\varepsilon_{11}^{H}-\varepsilon_{22}^{H}\right)}\right] .
$$

\section{Algorithmic aspects}

\subsection{Bifurcation criteria}

To predict the onset of necking using both bifurcation criteria, and to plot the corresponding necking limit strains in terms of forming limit diagrams (FLDs), the metal sheet is submitted to biaxial loading defined by the following form of velocity gradient $\mathbf{g}$ :

$$
\mathbf{g}=\mathbf{d}=\left[\begin{array}{ccc}
d_{11} & 0 & 0 \\
0 & \rho d_{11} & 0 \\
0 & 0 & d_{33}
\end{array}\right],
$$


where:

- $d_{11}$ is the major strain rate, which is set here to 1 all along loading. As the behavior is rateindependent, the value given to $d_{11}$ has no effect on the mechanical response.

- $\rho$ is the strain-path ratio, which ranges from $-1 / 2$ (uniaxial tension state) to 1 (equibiaxial tension state).

- the component $d_{33}$ is a priori unknown and is determined from the plane-stress condition $\left(\sigma_{33}=0\right)$.

The algorithm developed for the prediction of forming limit diagrams using both bifurcation criteria is based on two nested loops:

- For each strain-path ratio $\rho$ ranging between $-1 / 2$ and 1 (with $\Delta \rho=0.1$ ):

- For each time increment $\left[t_{n}, t_{n+1}\right]$ (with $t_{n+1}=t_{n}+\Delta t$ ), we apply the incremental algorithm detailed in Section 3.3 to integrate the constitutive equations and then to compute the analytical tangent modulus $\mathcal{L}$. Once $\mathcal{L}$ is computed, the condensed moduli $\tilde{\mathcal{L}}$ and $\tilde{\mathcal{L}}^{\text {p }}$ are determined by Eqs. (41) and (43), respectively. To apply the general bifurcation criterion, the eigenvalues of $\tilde{\mathcal{L}}_{\text {sym }}^{\text {p }}$ (symmetric part of $\tilde{\mathcal{L}}^{\text {p }}$ ) are computed. Diffuse necking is predicted when the smallest eigenvalue of $\tilde{\mathcal{L}}_{\text {sym }}^{\mathrm{p}}$ vanishes or, in an equivalent way, when $\tilde{\mathcal{L}}_{\text {sym }}^{\text {p }}$ becomes singular (as $\tilde{\mathcal{L}}_{\text {sym }}^{\text {p }}$ is positive definite before it becomes singular). To apply the Rice bifurcation criterion, the orientation $\theta$ providing the minimum value for $\operatorname{det}(\overrightarrow{\mathbf{v}} \cdot \tilde{\mathcal{L}} \cdot \overrightarrow{\mathbf{v}})$ over the interval $\left[0^{\circ}, 90^{\circ}\right]$ is searched. If this minimum value is negative, localized necking is predicted and the computation is stopped. As long as the conditions for necking are not met yet, the different mechanical variables are updated and the computation is continued for the next time increment.

\subsection{Initial imperfection approach}

When the initial imperfection approach is followed, the homogeneous zone of the sheet is submitted to the same loading as that prescribed for the whole sheet in Section 3.1. In this case, the algorithm for the determination of forming limit diagrams is mainly based on three nested loops:

- For $\rho=-1 / 2$ to $\rho=1$ at user-defined intervals (with $\Delta \rho=0.1$ ).

○ For $\theta_{0}$ spanning the admissible range of inclination angle between $0^{\circ}$ and $90^{\circ}$ (with $\left.\Delta \theta=1^{\circ}\right)$. 
- For each time increment $\left[t_{n}, t_{n+1}\right]$ (with $t_{n+1}=t_{n}+\Delta t$ ), apply the implicit incremental algorithm described in Section 3.3. The application of this incremental integration scheme is stopped when the following criterion is satisfied:

$$
d_{33}^{B} / d_{33}^{H} \geq 10 .
$$

The strain component $\varepsilon_{11}^{H}$, obtained when the criterion given by Eq. (52) is satisfied, is considered to be the critical strain $\varepsilon_{11}^{*}$ corresponding to the current band inclination $\theta$ and strain-path ratio $\rho$.

The smallest critical strain $\varepsilon_{11}^{*}$ solution of the above algorithm (over all possible initial angles $\theta_{0}$ ) and the corresponding current angle define, respectively, the necking limit strain $\varepsilon_{11}^{L}$ and the necking band orientation for the current strain-path ratio $\rho$.

In order to enhance the efficiency of the developed numerical tool, an adaptive time step technique is set up. With this strategy, the time step $\Delta t$ used in the internal loop for the incremental integration of the different equations is multiplied by a factor $\beta$ when the number of iterations for the last converged increment does not exceed $n$ iterations. In our computations, parameters $\beta$ and $n$ are set to 1.1 and 5 , respectively.

The different necking algorithms, presented in Sections 3.1 and 3.2, are implemented using the multiparadigm numerical computing environment Mathematica.

\subsection{Incremental algorithms}

The aim of the current section is to describe the incremental algorithms (over a typical time increment $\left.\left[t_{n}, t_{n+1}\right]\right)$ used to integrate the different equations that govern the coupling between the constitutive framework defined in Sections 2.1 and 2.2 and the necking criteria detailed in Section 2.3.

\subsubsection{Bifurcation criteria}

First, the set of incremental equations to be solved and the corresponding set of incremental unknowns are recalled:

- The plane-stress condition:

$$
\Delta \sigma_{33}=0
$$

The incremental unknown corresponding to this equation is $\Delta \varepsilon_{33}$.

- The normality law given by Eq. (6) leads to:

$$
\Delta \boldsymbol{\varepsilon}^{p s}=\Delta \gamma^{s} \frac{\partial \hat{\sigma}^{s}}{\partial \boldsymbol{\sigma}}, \quad \Delta \boldsymbol{\varepsilon}^{p t}=\Delta \gamma^{t} \frac{\partial \hat{\sigma}^{t}}{\partial \boldsymbol{\sigma}} .
$$


The incremental unknowns corresponding to these equations are $\Delta \boldsymbol{\varepsilon}^{p s}$ and $\Delta \boldsymbol{\varepsilon}^{p t}$. Under the plane-stress condition, and considering the incompressibility of plastic strain for each deformation mode, $\Delta \boldsymbol{\varepsilon}^{p s}$ and $\Delta \boldsymbol{\varepsilon}^{p t}$ can be generally expressed in the following generic forms:

$$
\begin{aligned}
\Delta \boldsymbol{\varepsilon}^{p s} & =\left[\begin{array}{ccc}
\Delta \varepsilon_{11}^{p s} & \Delta \varepsilon_{12}^{p s} & 0 \\
\Delta \varepsilon_{12}^{p s} & \Delta \varepsilon_{22}^{p s} & 0 \\
0 & 0 & -\left(\Delta \varepsilon_{11}^{p s}+\Delta \varepsilon_{22}^{p s}\right)
\end{array}\right], \\
\Delta \boldsymbol{\varepsilon}^{p t} & =\left[\begin{array}{ccc}
\Delta \varepsilon_{11}^{p t} & \Delta \varepsilon_{12}^{p t} & 0 \\
\Delta \varepsilon_{12}^{p t} & \Delta \varepsilon_{22}^{p t} & 0 \\
0 & 0 & -\left(\Delta \varepsilon_{11}^{p t}+\Delta \varepsilon_{22}^{p t}\right)
\end{array}\right] .
\end{aligned}
$$

- The Fischer-Burmeister conditions given by Eq. (17) lead to:

$$
\begin{aligned}
& \Psi^{s}=\sqrt{\left(\Phi^{s}\right)^{2}+\left(\Delta \gamma^{s}\right)^{2}}+\left(\Phi^{s}-\Delta \gamma^{s}\right)=0, \\
& \Psi^{t}=\sqrt{\left(\Phi^{t}\right)^{2}+\left(\Delta \gamma^{t}\right)^{2}}+\left(\Phi^{t}-\Delta \gamma^{t}\right)=0 .
\end{aligned}
$$

The incremental unknowns corresponding to these conditions are $\Delta \gamma^{s}$ and $\Delta \gamma^{t}$.

Summarizing the above relations, it appears that the governing equations for the sheet metal reduce to nine scalar equations: Eq. (53) (one scalar equation), Eq. (54) (six scalar equations) and Eq. (56) (two scalar equations). To solve these equations, nine scalar incremental unknowns need to be determined: $\Delta \varepsilon_{33}, \Delta \varepsilon_{11}^{p s}, \Delta \varepsilon_{12}^{p s}, \Delta \varepsilon_{22}^{p s}, \Delta \varepsilon_{11}^{p t}, \Delta \varepsilon_{12}^{p t}, \Delta \varepsilon_{22}^{p t}, \Delta \gamma^{s}$ and $\Delta \gamma^{t}$. The above equations are summarized in a set $\mathbf{Y}$ of nine scalar equations defined as follows:

$$
\begin{aligned}
& Y_{1 \rightarrow 5}=\left\{\Delta \sigma_{33}, \Delta \varepsilon_{11}^{p s}-\Delta \gamma^{s} \frac{\partial \hat{\sigma}^{s}}{\partial \sigma_{11}}, \Delta \varepsilon_{12}^{p s}-\Delta \gamma^{s} \frac{\partial \hat{\sigma}^{s}}{\partial \sigma_{12}}, \Delta \varepsilon_{22}^{p s}-\Delta \gamma^{s} \frac{\partial \hat{\sigma}^{s}}{\partial \sigma_{22}}, \Psi^{s}\right\}, \\
& Y_{6 \rightarrow 9}=\left\{\Delta \varepsilon_{11}^{p t}-\Delta \gamma^{t} \frac{\partial \hat{\sigma}^{t}}{\partial \sigma_{11}}, \Delta \varepsilon_{12}^{p t}-\Delta \gamma^{t} \frac{\partial \hat{\sigma}^{t}}{\partial \sigma_{12}}, \Delta \varepsilon_{22}^{p t}-\Delta \gamma^{t} \frac{\partial \hat{\sigma}^{t}}{\partial \sigma_{22}}, \Psi^{t}\right\} .
\end{aligned}
$$

The corresponding incremental unknowns are stored in a vector $\mathbf{X}$ defined as follows:

$$
\mathbf{X}=\left\{\Delta \varepsilon_{33}, \Delta \varepsilon_{11}^{p s}, \Delta \varepsilon_{12}^{p s}, \Delta \varepsilon_{22}^{p s}, \Delta \gamma^{s}, \Delta \varepsilon_{11}^{p t}, \Delta \varepsilon_{12}^{p t}, \Delta \varepsilon_{22}^{p t}, \Delta \gamma^{t}\right\}
$$

In summary, the incremental algorithm amounts to solving the following non-linear vector equation:

$$
\mathbf{Y}(\mathbf{X})=\mathbf{0} \text {. }
$$

This equation is implicitly solved using the predefined function 'FindRoot' of Mathematica. This function is based on an optimized implementation of the Newton-Raphson algorithm. Once the nonlinear system (59) is solved, the various mechanical variables are updated and the analytical modulus $\mathcal{L}$ is computed using Eq. (24). 


\subsubsection{Initial imperfection approach}

When the initial imperfection approach is used, the sheet metal is composed of the homogeneous zone and the band zone. The set of constitutive equations, very similar to the one formulated in Eq. (59), has to be developed for both zones:

$$
\mathbf{Y}^{H}\left(\mathbf{X}^{H}\right)=\mathbf{0}, \quad \mathbf{Y}^{B}\left(\mathbf{X}^{B}\right)=\mathbf{0} .
$$

Eq. (48) is added to Eq. (60) to obtain a complete set of incremental equations governing the initial imperfection approach. The unknowns to be determined from Eq. (48) are the two components of the jump vector $\dot{\overrightarrow{\mathbf{c}}}$. After having merged Eq. (48) with Eq. (60), one obtains the following global form of incremental equations:

$$
\breve{\mathbf{Y}}(\breve{\mathbf{X}})=\mathbf{0}
$$

where:

$$
\begin{aligned}
& \breve{Y}_{1 \rightarrow 5}=Y_{1 \rightarrow 5}^{H}=\left\{\Delta \sigma_{33}^{H}, \Delta \varepsilon_{11}^{p s H}-\Delta \gamma^{s H} \frac{\partial \hat{\sigma}^{s H}}{\partial \sigma_{11}}, \Delta \varepsilon_{12}^{p s H}-\Delta \gamma^{s H} \frac{\partial \hat{\sigma}^{s H}}{\partial \sigma_{12}}, \Delta \varepsilon_{22}^{p s H}-\Delta \gamma^{s H} \frac{\partial \hat{\sigma}^{s H}}{\partial \sigma_{22}}, \Psi^{s H}\right\}, \\
& \breve{Y}_{6 \rightarrow 9}=Y_{6 \rightarrow 9}^{H}=\left\{\Delta \varepsilon_{11}^{p t H}-\Delta \gamma^{t H} \frac{\partial \widehat{\sigma}^{t H}}{\partial \sigma_{11}}, \Delta \varepsilon_{12}^{p t H}-\Delta \gamma^{t H} \frac{\partial \widehat{\sigma}^{t H}}{\partial \sigma_{12}}, \Delta \varepsilon_{22}^{p t H}-\Delta \gamma^{t H} \frac{\partial \widehat{\sigma}^{t H}}{\partial \sigma_{22}}, \Psi^{t H}\right\}, \\
& \breve{Y}_{10 \rightarrow 14}=Y_{1 \rightarrow 5}^{B}=\left\{\Delta \sigma_{33}^{B}, \Delta \varepsilon_{11}^{p s B}-\Delta \gamma^{s B} \frac{\partial \widehat{\sigma}^{s B}}{\partial \sigma_{11}}, \Delta \varepsilon_{12}^{p s B}-\Delta \gamma^{s B} \frac{\partial \widehat{\sigma}^{s B}}{\partial \sigma_{12}}, \Delta \varepsilon_{22}^{p s B}-\Delta \gamma^{s B} \frac{\partial \widehat{\sigma}^{s B}}{\partial \sigma_{22}}, \Psi^{s B}\right\}, \\
& \breve{Y}_{15 \rightarrow 18}=Y_{6 \rightarrow 9}^{B}=\left\{\Delta \varepsilon_{11}^{p t B}-\Delta \gamma^{t B} \frac{\partial \widehat{\sigma}^{t B}}{\partial \sigma_{11}}, \Delta \varepsilon_{12}^{p t B}-\Delta \gamma^{t B} \frac{\partial \widehat{\sigma}^{t B}}{\partial \sigma_{12}}, \Delta \varepsilon_{22}^{p t B}-\Delta \gamma^{t B} \frac{\partial \hat{\sigma}^{t B}}{\partial \sigma_{22}}, \Psi^{t B}\right\}, \\
& \breve{Y}_{19 \rightarrow 20}=Y_{1 \rightarrow 2}^{M K}=\left\{\dot{c}_{1}-U_{1}, \dot{c}_{2}-U_{2}\right\} \text { where } \overrightarrow{\mathbf{U}}=\left(\overrightarrow{\mathbf{v}} \cdot \tilde{\mathcal{L}}^{B} \cdot \overrightarrow{\mathbf{v}}\right)^{-1}\left(\overrightarrow{\mathbf{v}} \cdot\left(\tilde{\mathcal{L}}^{H}-\xi \tilde{\mathcal{L}}^{B}\right): \tilde{\mathbf{g}}^{H}\right),
\end{aligned}
$$

and the set of unknown vectors is defined as follows:

$$
\begin{aligned}
& \breve{X}_{1 \rightarrow 9}=X_{1 \rightarrow 9}^{H}=\left\{\Delta \varepsilon_{33}^{H}, \Delta \varepsilon_{11}^{p s H}, \Delta \varepsilon_{12}^{p s H}, \Delta \varepsilon_{22}^{p s H}, \Delta \gamma^{s H}, \Delta \varepsilon_{11}^{p t H}, \Delta \varepsilon_{12}^{p t H}, \Delta \varepsilon_{22}^{p t H}, \Delta \gamma^{t H}\right\}, \\
& \breve{X}_{10 \rightarrow 18}=X_{1 \rightarrow 9}^{B}=\left\{\Delta \varepsilon_{33}^{B}, \Delta \varepsilon_{11}^{p s B}, \Delta \varepsilon_{12}^{p s B}, \Delta \varepsilon_{22}^{p s B}, \Delta \gamma^{s B}, \Delta \varepsilon_{11}^{p t B}, \Delta \varepsilon_{12}^{p t B}, \Delta \varepsilon_{22}^{p t B}, \Delta \gamma^{t B}\right\}, \\
& \breve{X}_{19 \rightarrow 20}=X_{1 \rightarrow 2}^{M K}=\left\{\dot{c}_{1}, \dot{c}_{2}\right\} .
\end{aligned}
$$

The above-described equations (i.e., twenty scalar incremental equations) are strongly non-linear and coupled. In fact, one cannot solve the equations corresponding to the band zone without determining the jump vector $\dot{\overrightarrow{\mathbf{c}}}$, because the velocity gradient in the band zone is dependent on this jump vector through the compatibility condition given by Eq. (45). Similarly, it is not possible to determine $\dot{\overrightarrow{\mathbf{c}}}$ from Eq. (48) without determining $\overrightarrow{\mathbf{v}}$ (which depends on strain components in the homogeneous zone, as shown in Eq. (50)), $\tilde{\mathcal{L}}^{H}$ (which depends on the mechanical state in the homogenous zone, as shown in Eq. (24)), $\tilde{\mathcal{L}}^{B}$ (which depends on the mechanical state in the band zone, as shown in Eq. (24)), $\xi$ (which depends on strain components in both the homogeneous zone and the safe one, as shown in Eq. (49)), and $\tilde{\mathbf{g}}^{H}$. To solve this set of equations, an iterative procedure, very similar to the one introduced 
in Section 3.3.1, shall be used. Once the different variables updated at $t_{n+1}$, the occurrence of localized necking is investigated by using criterion (52).

\section{Numerical predictions}

The material data required for the simulations reported in Sections 4.2 and 4.3 are summarized in Section 4.1. Then, the validation of the numerical algorithm proposed in Section 3.3 (implicit integration of the constitutive equations) is carried out in Section 4.2. The predictions of forming limits are presented in Section 4.3, where the associated numerical simulations are conducted using the material parameters given in Section 4.1. Also, Section 4.4 is devoted to a sensitivity study, where the impact of some material parameters on the prediction of the ductility limits is carefully analyzed. Finally, Section 4.5 focuses on the investigation of the effect of distortional hardening on the prediction of the ductility limits using the different necking criteria.

\subsection{Material data and initial imperfection factor}

The hardening yield functions for the AZ31 magnesium alloy used in the simulations shown in Sections 4.2, 4.3 and 4.4 are defined as follows (Steglich et al., 2016):

$$
\begin{aligned}
& R^{s}\left(\gamma^{s}, \gamma^{t}\right)=R_{0}^{s}+H^{s t} \gamma^{t}+Q_{1}^{s}\left(1-\exp \left(-b_{1}^{s} \gamma^{s}\right)\right)+Q_{2}^{s}\left(1-\exp \left(-b_{2}^{s} \gamma^{s}\right)\right), \\
& R^{t}\left(\gamma^{s}, \gamma^{t}\right)=R_{0}^{t}+H^{t} \gamma^{t}+Q_{1}^{t}\left(\exp \left(b_{1}^{t} \gamma^{t}\right)-1\right)+Q_{2}^{t}\left(1-\exp \left(-b_{2}^{t} \gamma^{s}\right)\right) .
\end{aligned}
$$

Consequently, the derivatives $\partial R^{s} / \partial \gamma^{s}, \partial R^{s} / \partial \gamma^{t}, \partial R^{t} / \partial \gamma^{s}$ and $\partial R^{t} / \partial \gamma^{t}$ required for the computation of $M^{s s}, M^{s t}, M^{t s}$ and $M^{t t}$ are given by the following relations:

$$
\begin{aligned}
& \frac{\partial R^{s}}{\partial \gamma^{s}}=Q_{1}^{s} b_{1}^{s} \exp \left(-b_{1}^{s} \gamma^{s}\right)+Q_{2}^{s} b_{2}^{s} \exp \left(-b_{2}^{s} \gamma^{s}\right), \quad \frac{\partial R^{s}}{\partial \gamma^{t}}=H^{s t}, \\
& \frac{\partial R^{t}}{\partial \gamma^{s}}=Q_{2}^{t} b_{2}^{t} \exp \left(-b_{2}^{t} \gamma^{s}\right), \quad \frac{\partial R^{t}}{\partial \gamma^{t}}=H^{t}+Q_{1}^{t} b_{1}^{t} \exp \left(b_{1}^{t} \gamma^{t}\right) .
\end{aligned}
$$

The material parameters corresponding to these simulations have been identified by Steglich et al. (2016) and are listed in Table 1. For these simulations, distortional hardening is neglected as all the anisotropy parameters are taken to be constant during plastic loading. 
Table 1. Material parameters for the AZ31 magnesium alloy (stress-like parameters are expressed in MPa).

\begin{tabular}{|c|c|c|c|c|c|c|c|}
\hline \multirow{2}{*}{ Elasticity } & & \multicolumn{3}{|l|}{$E$} & & & \\
\hline & & 43000 & 0.29 & & & & \\
\hline \multirow{6}{*}{ Slip } & \multirow{2}{*}{ hardening } & $R_{0}^{s}$ & $H^{s t}$ & $Q_{1}^{s}$ & $b_{1}^{s}$ & $Q_{2}^{s}$ & $b_{2}^{s}$ \\
\hline & & 145.3 & 1984.1 & 178.8 & 24.8 & 78.2 & 636.8 \\
\hline & \multirow{4}{*}{ anisotropy } & $l_{11}^{s}$ & $l_{22}^{s}$ & $l_{33}^{s}$ & $l_{12}^{s}$ & $l_{23}^{s}$ & $l_{13}^{s}$ \\
\hline & & 1.06 & 1.58 & 0.898 & 1.41 & 1 & 1 \\
\hline & & $l_{44}^{s}$ & $l_{55}^{s}$ & $l_{66}^{s}$ & $a^{s}$ & & \\
\hline & & 1 & 1 & 1 & 7.81 & & \\
\hline \multirow{6}{*}{ Twinning } & \multirow{2}{*}{ hardening } & $R_{0}^{t}$ & $H^{t}$ & $Q_{1}^{t}$ & $b_{1}^{t}$ & $Q_{2}^{t}$ & $b_{2}^{t}$ \\
\hline & & 75.0 & 470.1 & 2.38 & 54.1 & 0 & 0 \\
\hline & \multirow{4}{*}{ anisotropy } & $l_{11}^{t}$ & $l_{22}^{t}$ & $l_{33}^{t}$ & $l_{12}^{t}$ & $l_{23}^{t}$ & $l_{13}^{t}$ \\
\hline & & 1.4 & 0.013 & 1.41 & 0.077 & 0.121 & 0.664 \\
\hline & & $l_{44}^{t}$ & $l_{55}^{t}$ & $l_{66}^{t}$ & $a^{t}$ & $k$ & \\
\hline & & 1 & 1 & 0.696 & 2.98 & 0.91 & \\
\hline
\end{tabular}

The initial imperfection factor $f_{0}$, which is equal to $1-e_{0}^{B} / e_{0}^{A}$ (Fig. 2), is set to $5 \times 10^{-3}$ for all the simulations based on the M-K approach (except for the results of Fig. 6c, where the effect of $\xi_{0}$ on the shape and the level of the forming limit diagrams is analyzed). It should be mentioned that $f_{0}$ usually ranges from $10^{-3}$ to $2 \times 10^{-2}$. In the different figures presented in what follows, the stress components $\left(\sigma_{11}, \sigma_{22}, F / S_{0} \ldots\right)$ are expressed in $\mathrm{MPa}$.

\subsection{Validation of the numerical implementation}

In a preliminary stage, several numerical simulations are carried out to evaluate the accuracy of the numerical implementation of the constitutive equations with the implicit algorithm developed in Section 3.3. This evaluation is based on a number of relevant comparisons, using the parameters identified in Steglich et al. (2016), in order to assess the numerical implementation of the constitutive framework. The results of these simulations are presented in Fig. 3. Despite the complexity of the material behavior, the accuracy of the developed implicit integration scheme is clearly confirmed in Fig. 3a, where a uniaxial compression test is simulated with several strain increments $\Delta \varepsilon_{11}$. The results of this figure show that the compression stress-strain curve is almost insensitive to the strain increment size. Hence, the implemented implicit algorithm provides good accuracy even when large time increments are used. Another interesting consequence of this accuracy assessment is that the use 
of the adaptive time step strategy, embedded in the algorithm developed in Section 3.2, allows the CPU time to be considerably reduced without loss of accuracy. To further validate our numerical implementation, comparisons between the initial yield surfaces predicted by the developed numerical tool (dotted curves) and their counterparts determined by the analytical expressions given by Eqs. (8) and (12) (solid curves) are presented in Fig. 3b. This figure confirms that the different yield criteria are correctly implemented in the developed numerical tool. The comparisons between our results and those published by Steglich et al. (2016) are given in Fig. 3c and Fig. 3d. Fig. 3c shows the evolution of the nominal stress as a function of the nominal strain, as obtained by applying a uniaxial tensile test in the longitudinal direction (which coincides here with the 1 direction). As to Fig. 3d, it depicts the evolution of the relative width reduction as a function of the nominal strain for the same test. The results of Fig. $3 \mathrm{c}$ and Fig. $3 \mathrm{~d}$ reveal that the predictions obtained by the developed implicit algorithm are in excellent agreement with those reported in Steglich et al. (2016).

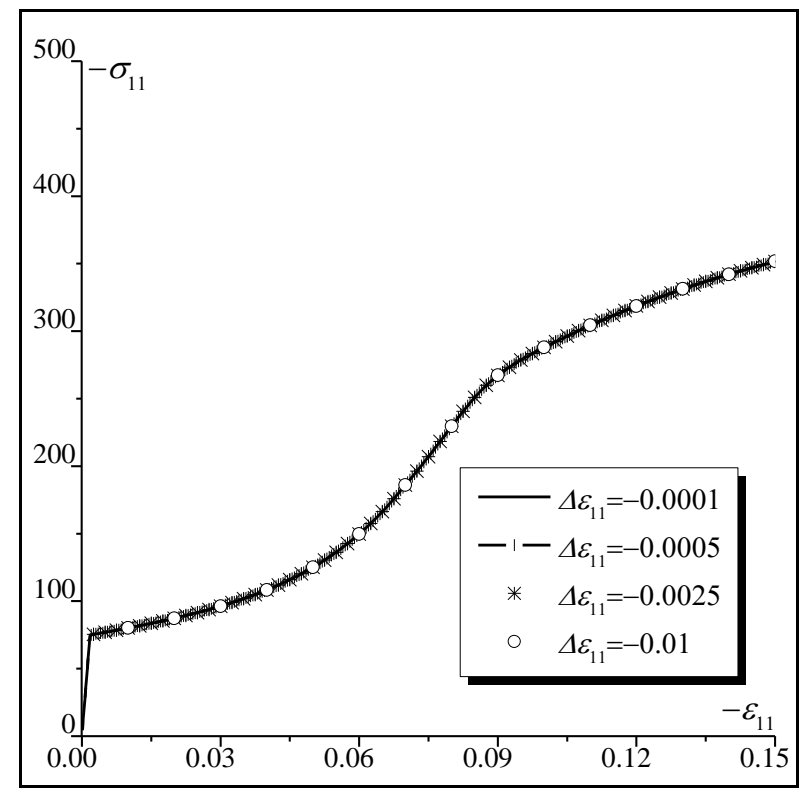

(a)

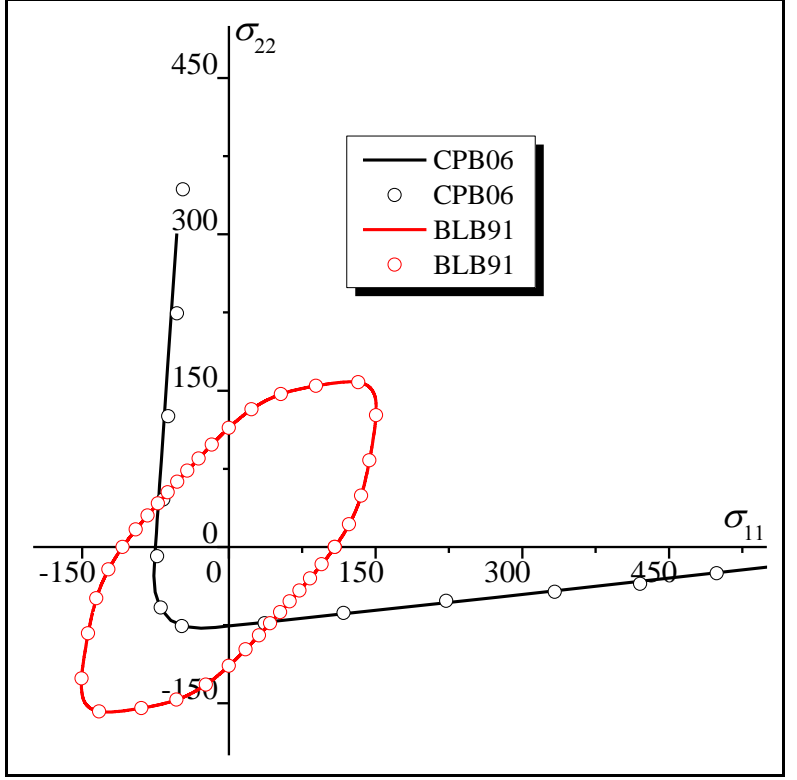

(b) 


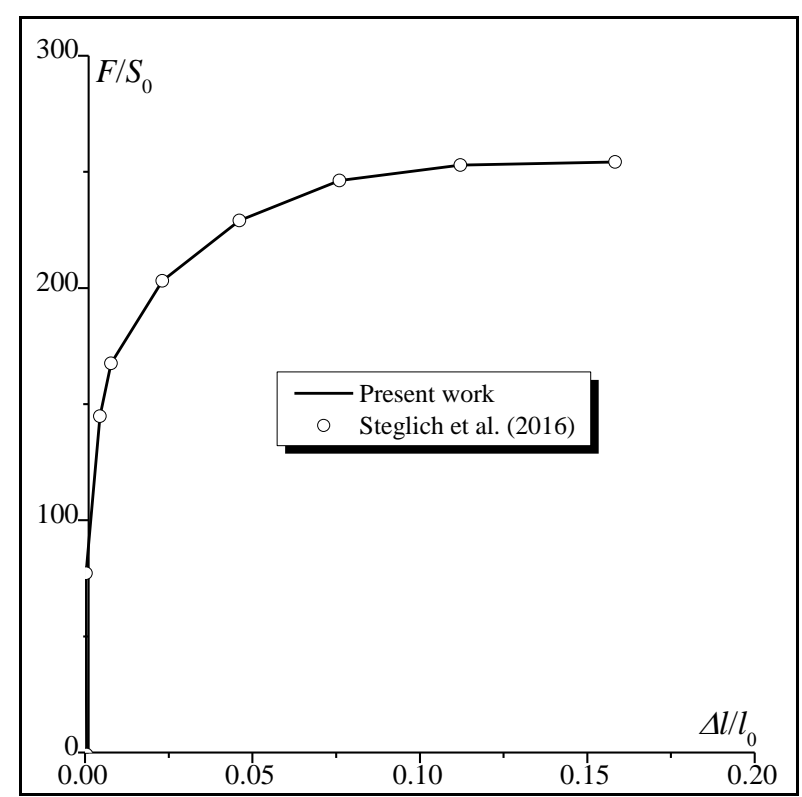

(c)

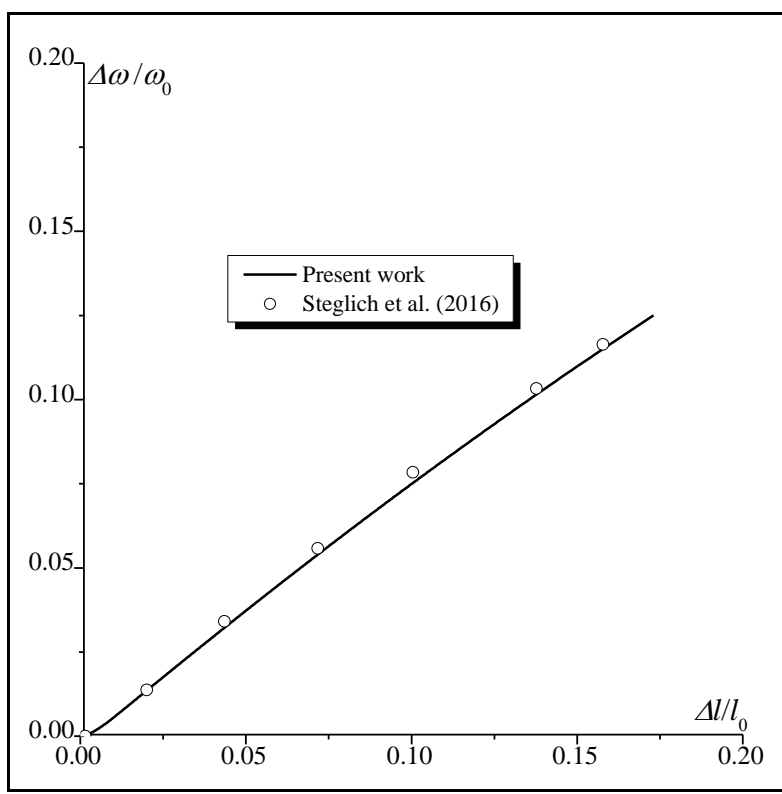

(d)

Fig. 3. Validation of the integration algorithm of the constitutive equations: (a) Accuracy of the numerical integration; (b) Validation of the implementation of the yield surfaces; (c) Comparison with results reported in Steglich et al. (2016): tension and compression stress-strain curves; (d) Comparison with results reported in Steglich et al. (2016): Lateral strain versus axial strain in tension.

\subsection{Prediction of forming limits}

The parameters given in Table 1 (Section 4.1) are used in this section to predict the mechanical behavior and the onset of necking in thin sheets. The evolutions of the accumulated slip $\gamma^{s}$ and twinning $\gamma^{t}$, as functions of the strain component $\varepsilon_{11}$, are displayed in Fig. 4a for two particular strain paths: the uniaxial tension strain path $(\rho=-0.5)$ and the equibiaxial tension strain path $(\rho=1)$. This figure clearly shows that the twinning mode is not activated for both strain paths (accordingly, the corresponding accumulated twinning remains equal to 0 all along loading), and that plasticity is only due to the activation of the slip mode. To further analyze this point, we plot in Fig. $4 \mathrm{~b}$ the initial yield surfaces and the yield surfaces corresponding to bifurcation (predicted by the GBC) for the uniaxial tensile strain path. The initial tensile state and the tensile state at bifurcation are represented by symbol 'o'. Starting from a stress-free state $\left(\sigma_{11}=\sigma_{22}=0\right)$ and following a uniaxial tension strain path, the inner yield surface determines the first active plastic deformation mode. In this case, the BLB91 yield surface is firstly reached. With increasing the equivalent slip strain $\gamma^{s}$, the material hardens and the BLB91 yield surface expands. On the other hand, the twinning yield surface remains unchanged, as the hardening parameter $Q_{2}^{t}$, which reflects the effect of the slip deformation mode on the evolution of the twinning yield function, is set to 0 . Despite the increase of $R^{s}$ all along the plastic deformation, the BLB91 yield surface does not intersect the CPB06 yield surface and hence the twinning mode is 
not activated. Consequently, the initial and the current (at bifurcation) twinning yield surfaces are identical. The same observation can be made for the equibiaxial tension strain path.

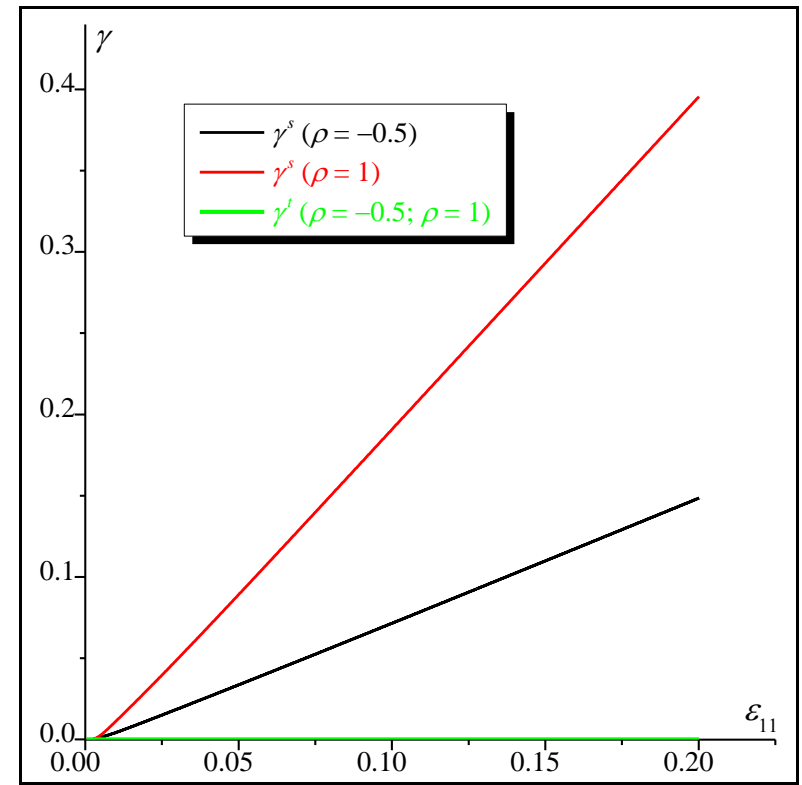

(a)

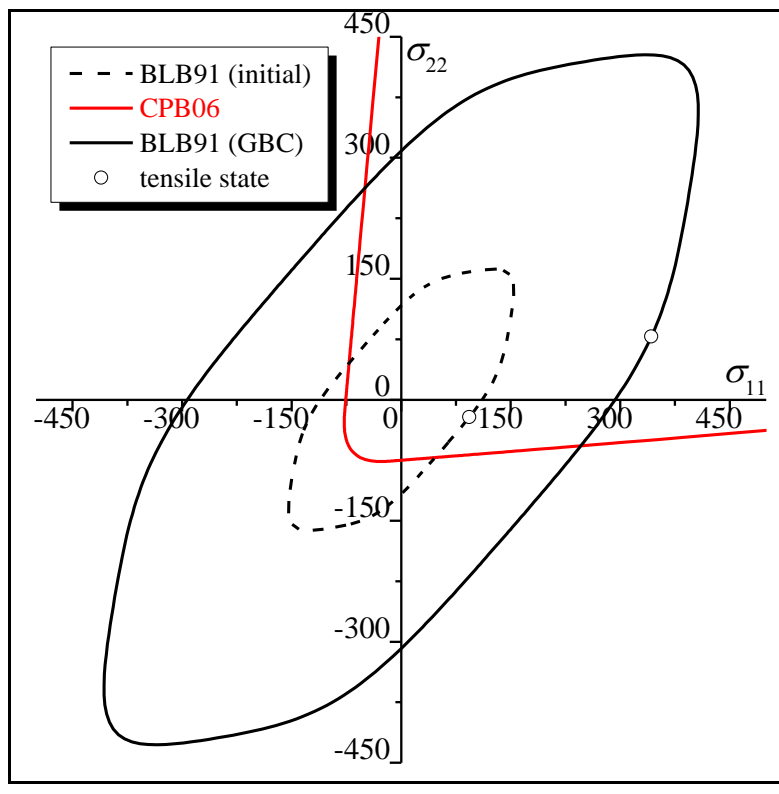

(b)

Fig. 4. Activation of the plastic deformation mode: (a) Evolution of the accumulated slip $\gamma^{s}$ and twinning $\gamma^{t}$ as functions of $\varepsilon_{11}$; (b) Evolution of the slip and twinning yield surfaces.

A preliminary study on the occurrence of plastic instability is undertaken and the corresponding results are shown in Fig. 5. Four particular strain-path ratios are selected for this preliminary analysis: $\rho=-0.5, \rho=0, \rho=0.5$ and $\rho=1$. The evolution of the cubic root of the determinant of $\tilde{\mathcal{L}}_{\text {sym }}^{\text {p }}$ (which has the same sign as the determinant of $\tilde{\mathcal{L}}_{\text {sym }}^{\text {p }}$ ) versus the major strain component $\varepsilon_{11}$ is plotted in Fig. 5a. This figure clearly shows that diffuse necking is predicted for the different strain paths at realistic strain levels (when $\tilde{\mathcal{L}}_{\text {sym }}^{\mathrm{p}}$ becomes singular). By contrast, the Rice bifurcation criterion does not predict strain localization for positive strain-path ratios (i.e., biaxial tension), as shown in Fig. 5b. This result is expectable and may be explained by the absence of key destabilizing effects within the adopted constitutive modeling. The results obtained by the M-K approach are reported in Fig. 5c. In this figure, localized necking is marked by the rapid increase of the plastic strain in the band as compared to the homogeneous zone. More specifically, localized necking occurs when the ratio $d_{33}^{B} / d_{33}^{H}$ becomes larger than 10 , as stated in Eq. (52), for a given strain path and initial band orientation. Fig. $5 \mathrm{c}$ shows that the presence of an initial geometric imperfection allows predicting localized necking at realistic strain levels for all of the investigated strain paths (even in the range of biaxial tension). 


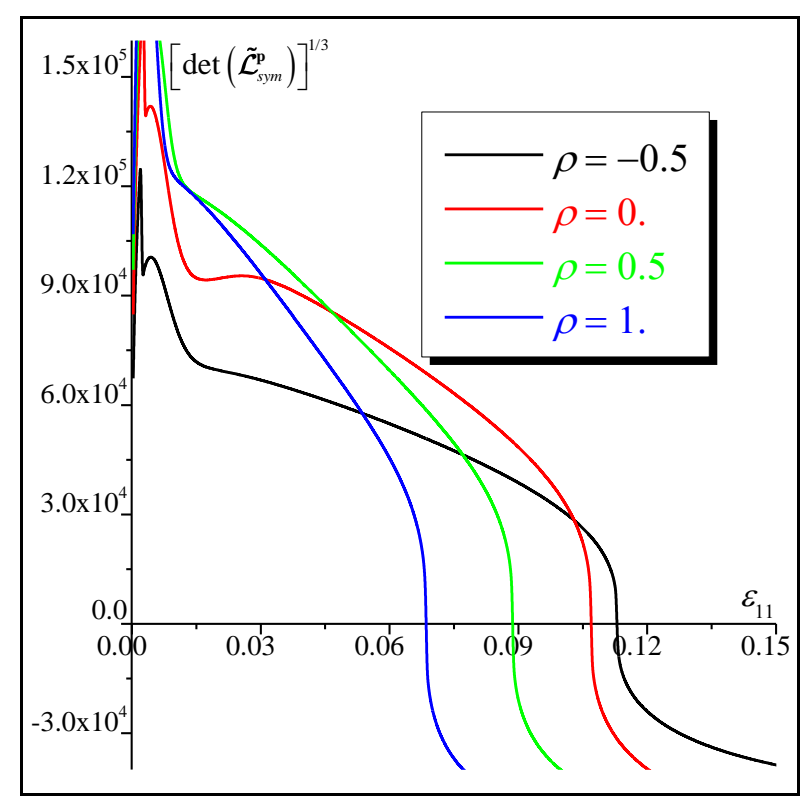

(a)

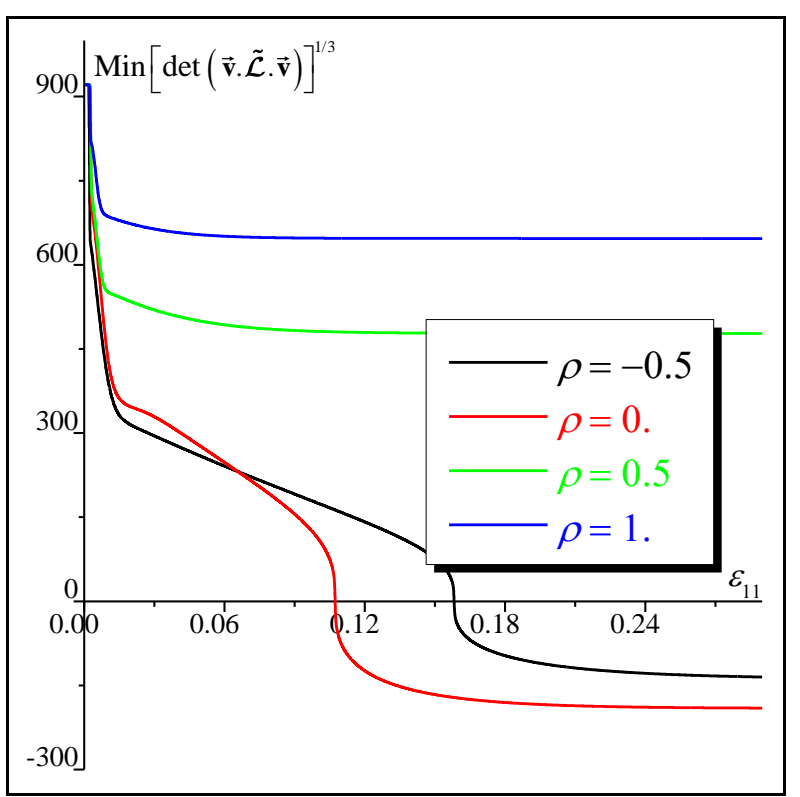

(b)

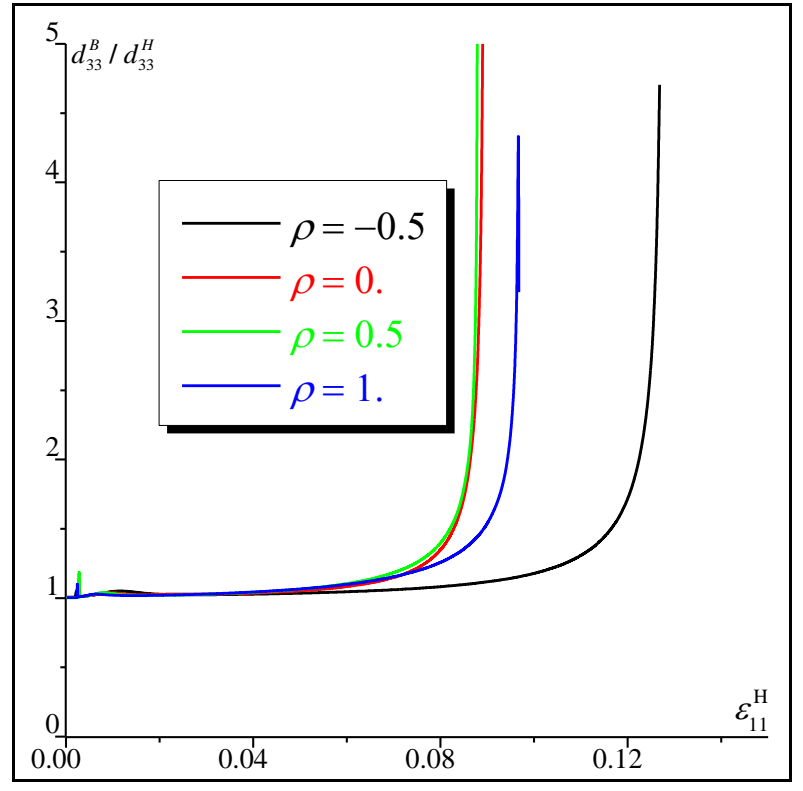

(c)

Fig. 5. Evolution of: (a) the cubic root of the determinant of $\tilde{\mathcal{L}}_{\text {sym }}^{\text {p }}$ versus $\varepsilon_{11}$ (general bifurcation criterion); (b) the minimum of the cubic root of the determinant of the acoustic tensor versus $\varepsilon_{11}$ (Rice bifurcation analysis);

(c) the localization indicator $d_{33}^{B} / d_{33}^{H}$ versus $\varepsilon_{11}^{H}$ (initial imperfection approach).

The Rice bifurcation criterion and the initial imperfection approach are used to plot the current orientation $\theta$ of the necking band versus the strain-path ratio $\rho$ (see Fig. 6a). As can be seen in this figure, the two localized necking criteria predict the same band orientation. Furthermore, the necking band remains perpendicular to the major strain direction when the strain-path ratio $\rho$ is greater than 
0.1. The FLDs predicted by the adopted bifurcation criteria are plotted in Fig. 6b. Upon close inspection of Fig. 6b, several observations are noted, which are worthy of further discussion. Firstly, diffuse necking predicted by the GBC and localized necking predicted by the RBC occur almost simultaneously for the plane-strain tension loading path $(\rho=0)$. For the other strain paths $(\rho \neq 0)$, diffuse necking occurs prior to localized necking, which is not reached at realistic strain levels for a wide range of positive strain paths $(\rho>0)$. Contrary to the $\mathrm{RBC}$, the initial imperfection approach allows predicting localized necking limit strains at realistic strain levels for the whole range of strain paths; however, these limit strains are mainly dependent on the value of the initial imperfection factor $f_{0}$ (Fig. $6 \mathrm{c}$ ). Note that when the size of the initial imperfection $f_{0}$ tends to zero, the FLDs predicted by the $\mathrm{M}-\mathrm{K}$ analysis tend towards the FLD predicted by the Rice bifurcation criterion. This observation is fully consistent considering the similarity of the mathematical formulations of the two approaches (Rice bifurcation and $\mathrm{M}-\mathrm{K}$ ), as shown by Eq. (48): if the amount of initial imperfection is set to zero in the imperfection model, the problem is reduced to the Rice bifurcation analysis.

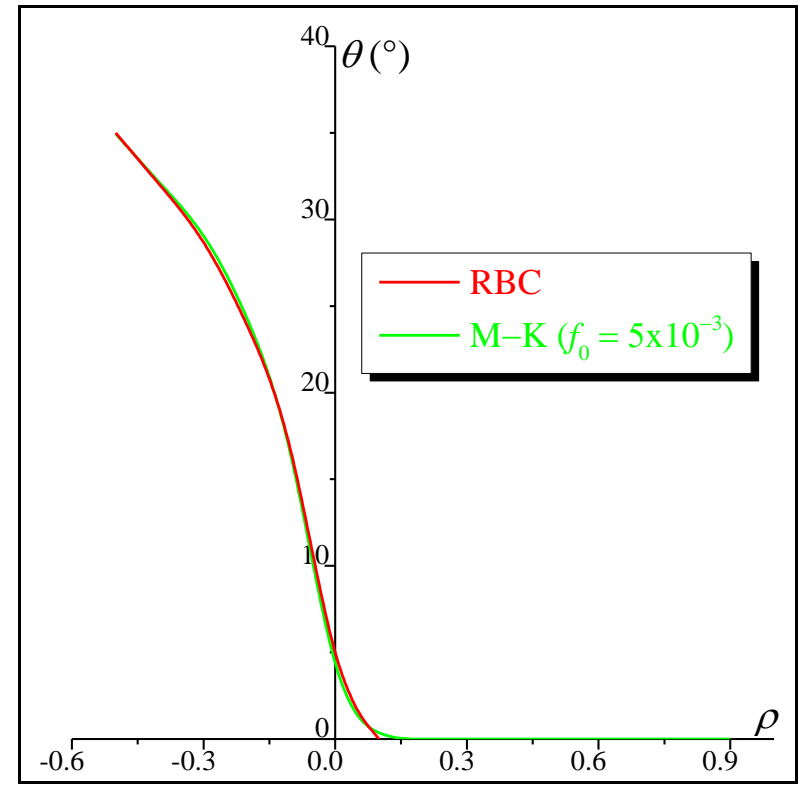

(a)

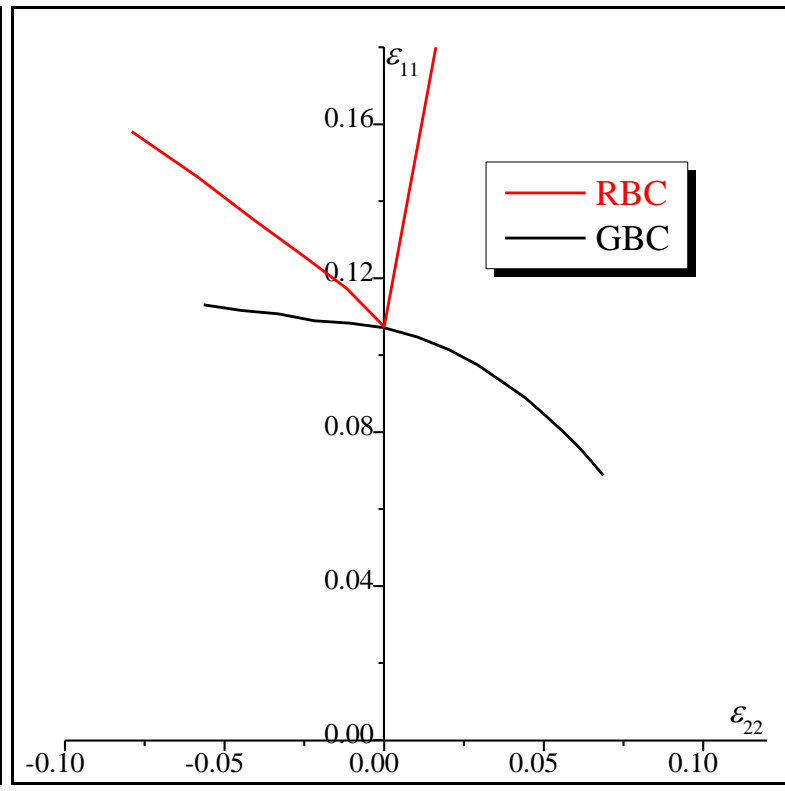

(b) 


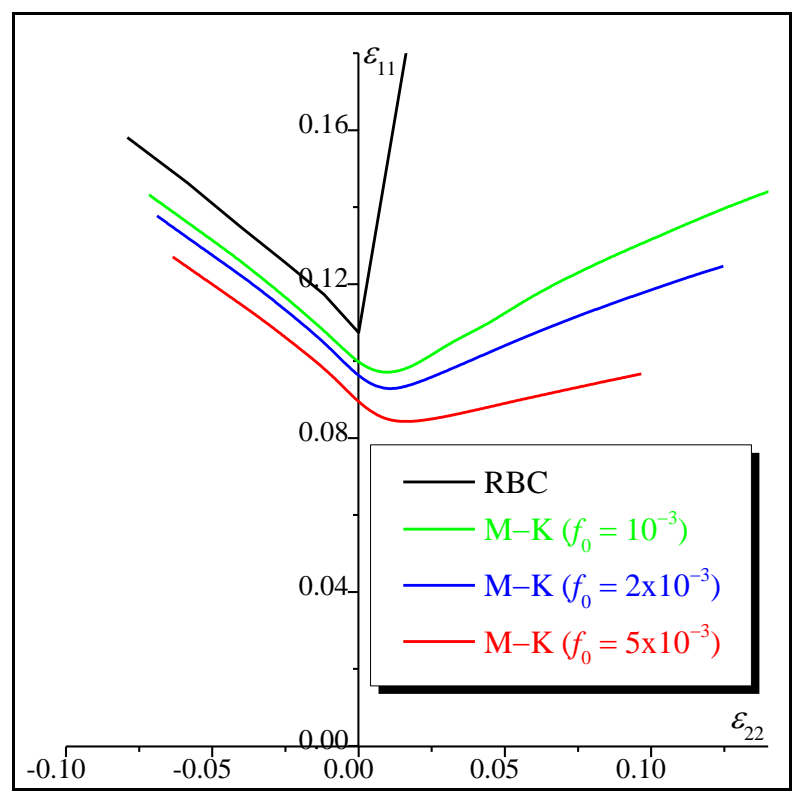

(c)

Fig. 6. Comparisons between the predictions obtained by the three plastic instability criteria for AZ31 magnesium alloy: (a) Evolution of the localization band orientation; (b) Forming limit diagrams predicted by the two bifurcation criteria (namely, RBC and GBC); (c) Forming limit diagrams predicted by the RBC and the $\mathrm{M}-\mathrm{K}$ approach with several initial imperfection ratios.

\subsection{Sensitivity study}

As discussed in the previous section, using the material parameters given in Table 1, the numerical predictions reveal that the twinning mode is not activated for strain paths ranging from uniaxial to equibiaxial tensile states. This observation is mainly due to the large value of the strength differential parameter $k$ involved in the Cazacu model (Eq. (12)). In fact, the use of such a large value for parameter $k$ results in a very high initial twinning yield stress for the domain $\left(\sigma_{11} \geq 0, \sigma_{22} \geq 0\right)$ as shown in Fig. 4b. A parameter sensitivity study is conducted to further investigate the impact of this asymmetry parameter on the mechanical behavior, in general, and on the prediction of plastic instability, in particular. In order to achieve this sensitivity analysis, we have used two other values for parameter $k: 0.5$ and 0.2 . The effect of this parameter on the shape and the evolution of the yield stress is illustrated in Fig. 7, which shows that a decrease of parameter $k$ allows a significant decrease of the twinning yield stress for the quadrant $\left(\sigma_{11} \geq 0, \sigma_{22} \geq 0\right)$. In this figure, the uniaxial tensile states at the beginning of plastic deformation and at bifurcation (predicted by the GBC) are represented by symbol 'o'. Unlike predictions obtained with $k=0.91$ (Fig. 4b), both slip and twinning modes are simultaneously activated before bifurcation when parameter $k$ is set to 0.5 or 0.2 . In these latter cases ( $k=0.5 ; 0.2$ ), the slip mode is firstly activated and subsequently the slip yield surface expands and meets the twinning yield surface. As for the case of uniaxial tensile state, slip and twinning modes are 
simultaneously activated before the occurrence of bifurcation for the other strain paths ranging between -0.5 and 1 when parameter $k$ is set to 0.5 or 0.2 .

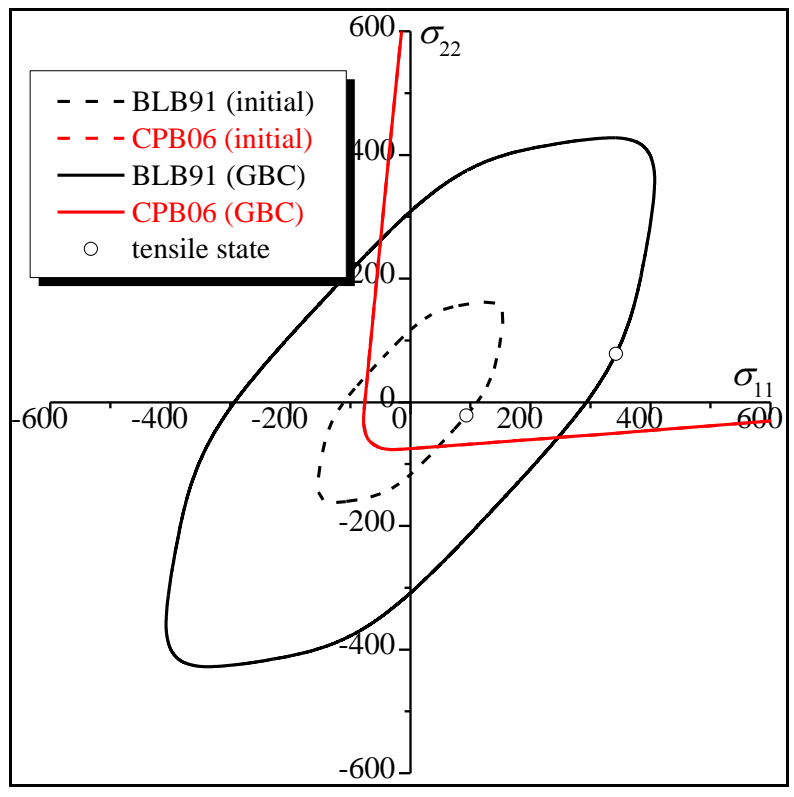

(a)

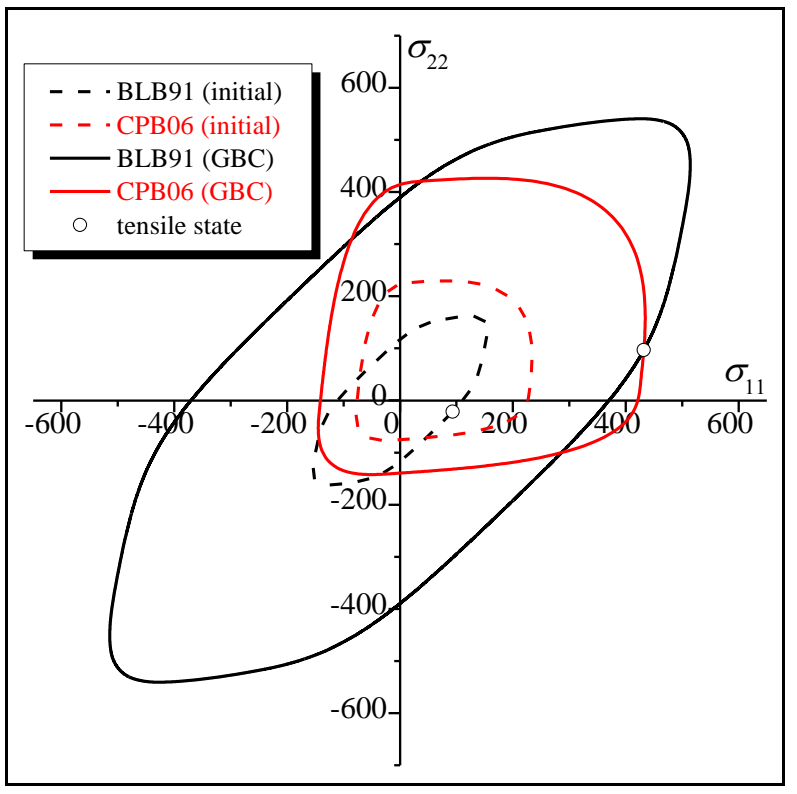

(b)

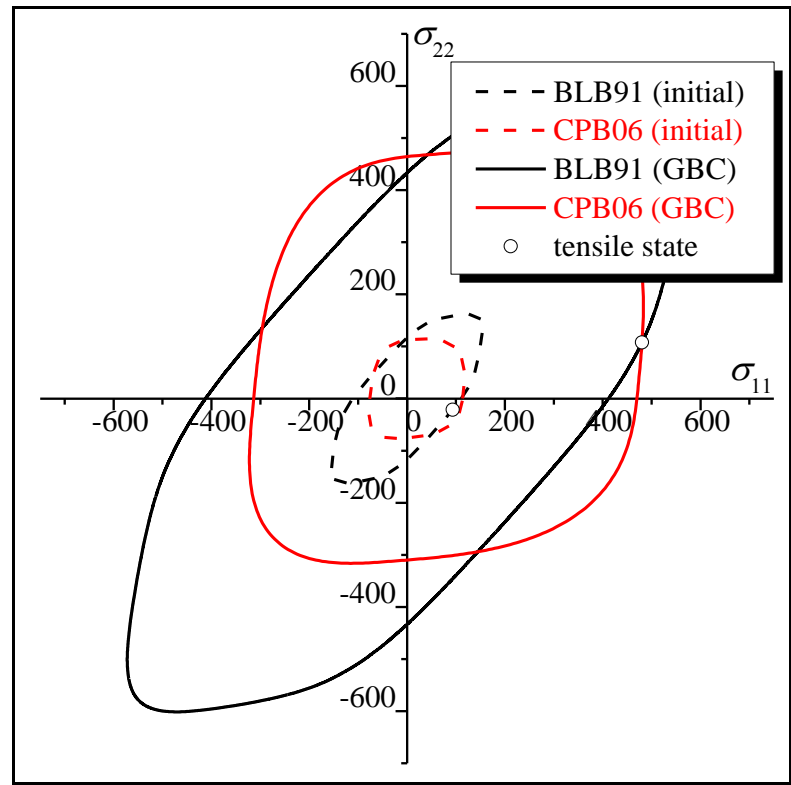

(c)

Fig. 7. Effect of the asymmetry parameter $k$ on the evolution of the yield surfaces: (a) $k=0.91$; (b) $k=0.5$; (c)

$$
k=0.2 .
$$

The effect of the strength differential parameter $k$ on the activation of the different plastic deformation modes is further investigated in Fig. 8. The curves of Fig. 8 confirm the results displayed in Fig. 7 stating that when parameter $k$ is set to 0.91 , only the slip mode (solid curves) is activated for the different studied strain paths. By contrast, twinning mode (dashed curves) is activated when $k$ is 
set to 0.2 or 0.5 with earlier activation for $k=0.2$. The activation of both deformation modes may likely be correlated with the level of the predicted ductility limits, as will be shown in Fig. 9.

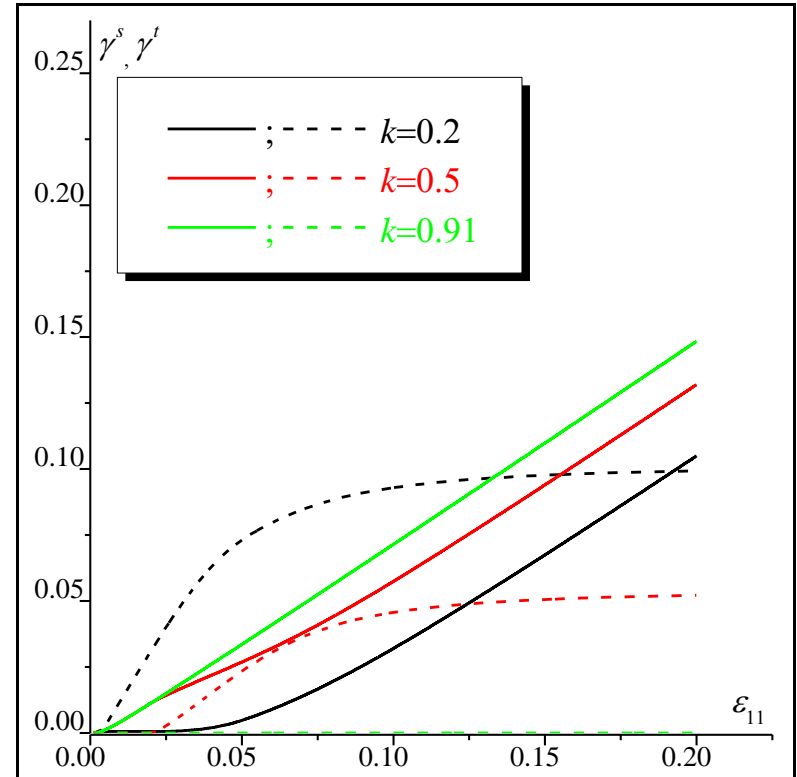

(a)

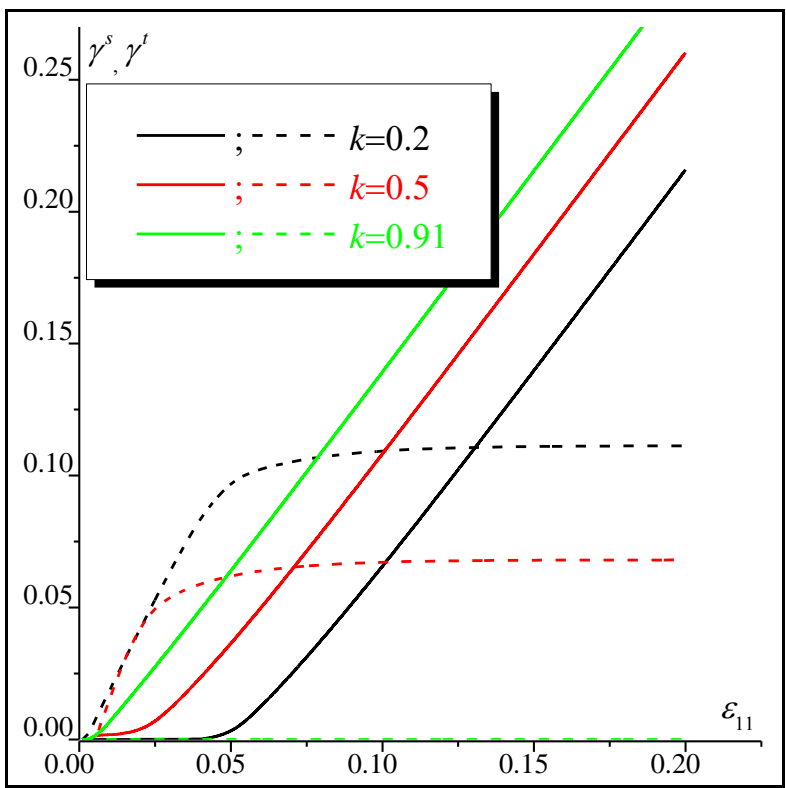

(c)

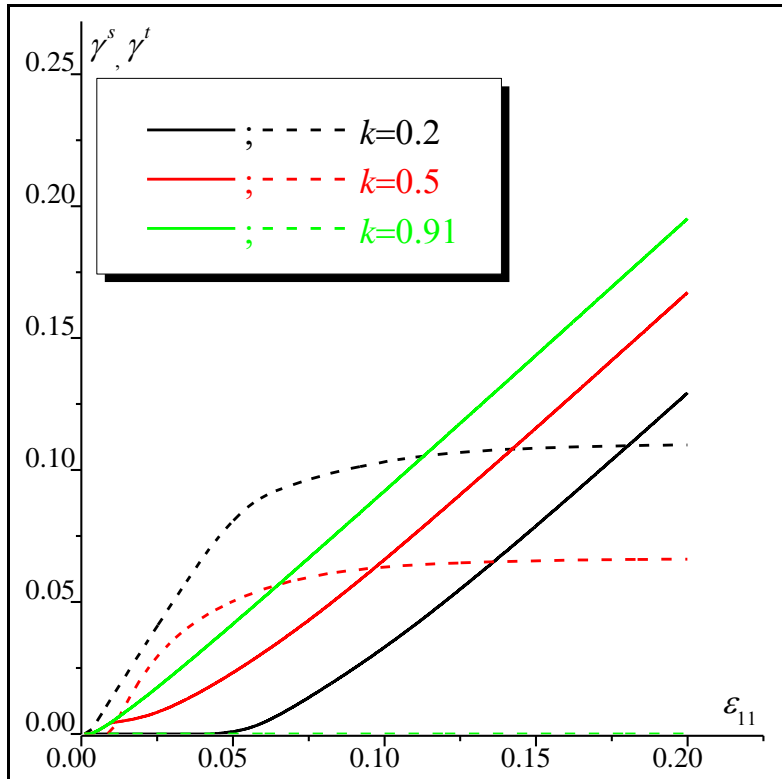

(b)

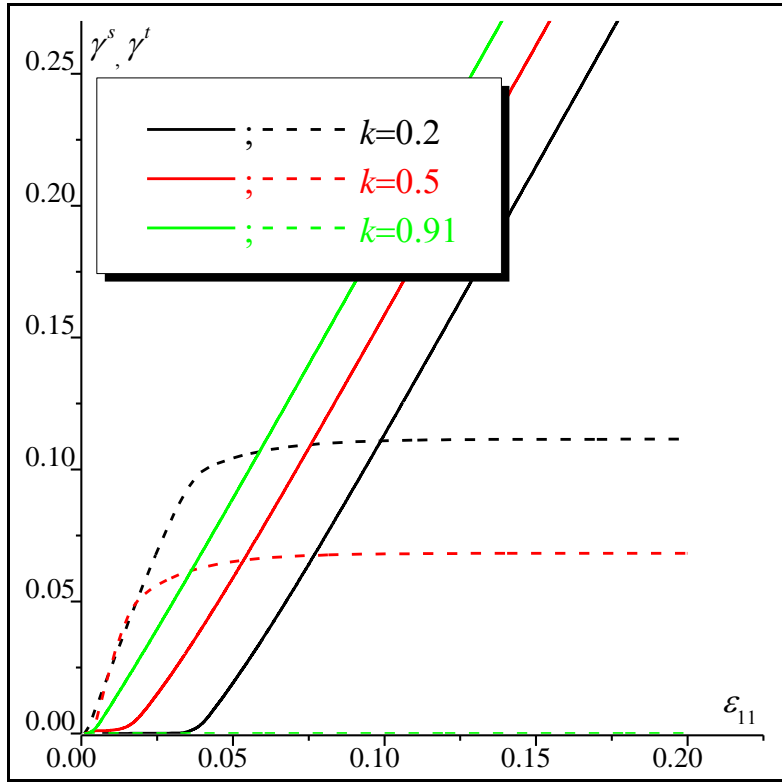

(d)

Fig. 8. Effect of the asymmetry parameter $k$ on the accumulated slip $\gamma^{s}$ and twinning $\gamma^{t}$ : (a) $\rho=-0.5 ;$ (b)

$$
\rho=0 ; \text { (c) } \rho=0.5 ; \text { (d) } \rho=1 \text {. }
$$

The effect of the asymmetry parameter $k$ on the different instability indicators (i.e., the cubic root of the determinant of $\tilde{\mathcal{L}}_{\text {sym }}^{\mathrm{p}}$, the minimum of the cubic root of the determinant of the acoustic tensor $\overrightarrow{\mathbf{v}} . \tilde{\mathcal{L}} \cdot \overrightarrow{\mathbf{v}}$, and the ratio $\left.d_{33}^{B} / d_{33}^{H}\right)$ as well as on the shape and the level of the predicted forming limit diagrams is shown in Fig. 9. This figure clearly demonstrates that the predicted necking limit strains increase when the value of the asymmetry parameter $k$ decreases. This observation is mainly due to 
the activation of the twinning mode for small values of $k(0.5$ and 0.2$)$. In fact, the activation of this mode, in addition to the slip mode, significantly modifies the shape of the resulting yield surface (as illustrated in Fig. 7) as well as the hardening evolution. It is well known that the onset of plastic instability is strongly dependent on these two key mechanical characteristics among other material parameters (such as strain-rate sensitivity). The simulations of Fig. 9 clearly illustrate the competition between hardening and plastic anisotropy effects in the prediction of diffuse and localized necking. On the one hand, the simultaneous activation of slip and twinning modes leads to a sharper yield locus (compared to the case of activation of slip mode only) and consequently promotes the occurrence of localized necking, especially in the range of positive strain paths (Barlat, 1987). However, the effect of such a curvature of the yield surface on the prediction of diffuse necking limit strains is very small, especially in the biaxial loading range, as has been demonstrated in several contributions (see, for instance, Kim et al., 2005; Zajkani and Bandizaki, 2017). On the other hand, the activation of the twinning deformation mode in addition to the slip mode leads to a significant increase in the evolution of the hardening modulus (defined as the variation of the yield stress with respect to plastic deformation). As a result, diffuse and localized necking limit strains increase with this hardening modulus. To further analyze the effect of the activation of the twinning deformation mode on the evolution of the hardening modulus, the equivalent von Mises stress $\sigma_{e q}$ is plotted versus the equivalent plastic strain $\varepsilon_{e q}^{p}$ in Fig. 10. These equivalent measures are defined as follows:

$$
\begin{aligned}
& \sigma_{e q}=\sqrt{(3 / 2) \sigma_{d}: \sigma_{d}} \text { where } \sigma_{d}=\boldsymbol{\sigma}-(\operatorname{tr}(\boldsymbol{\sigma}) / 3) \mathbf{I}_{2}, \\
& \varepsilon_{e q}^{p}=\int_{0}^{t} d_{e q}^{p} d t \text { where } d_{e q}^{p}=\sqrt{(2 / 3) \mathbf{d}^{p}: \mathbf{d}^{p}} \text { and } \mathbf{d}^{p}=\mathbf{d}^{p s}+\mathbf{d}^{p t},
\end{aligned}
$$

In the same figure, the evolution of the hardening modulus $h=d \sigma_{e q} / d \varepsilon_{e q}^{p}$ is plotted as a function of the equivalent plastic strain. The predictions presented in Fig. 10 clearly demonstrate that the activation of the twinning mode results in a significant increase in the magnitude of the hardening modulus. This evolution is likely to be linked to the expressions of the yield stresses defined in Eq. (64) and the corresponding hardening parameters reported in Table 1. Consequently, the activation of the twinning mode leads to an increase in the necking limit strains (predicted by both diffuse and localized necking criteria), as shown in Fig. 9, despite the fact that the resulting yield surface is sharper than the one corresponding to the activation of the slip mode only. In summary, in this particular context, the effect of hardening on the level of predicted limit strains is more important than the effect of the yield surface curvature. 


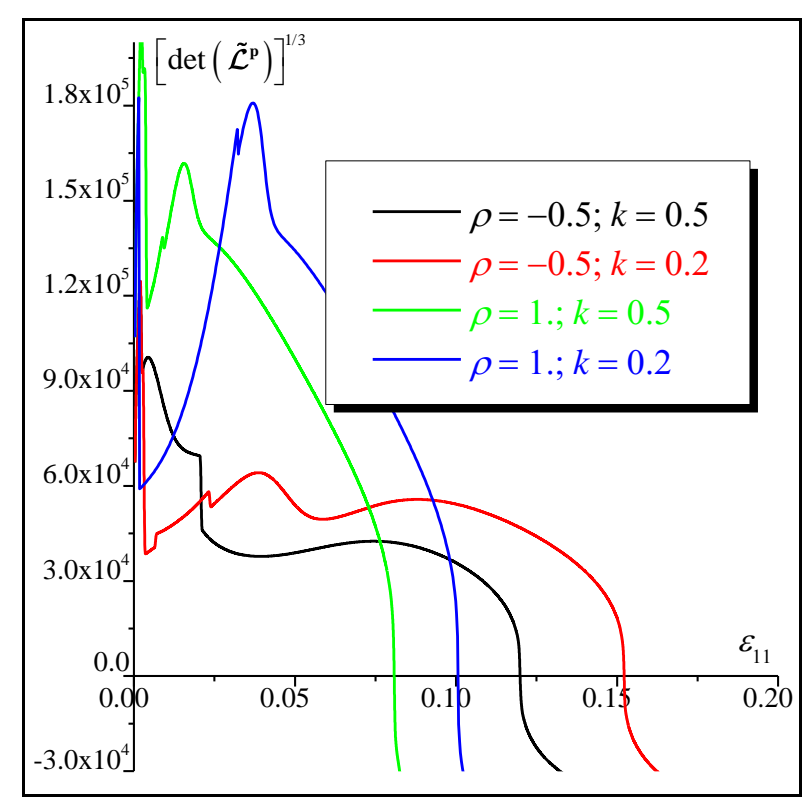

(a)

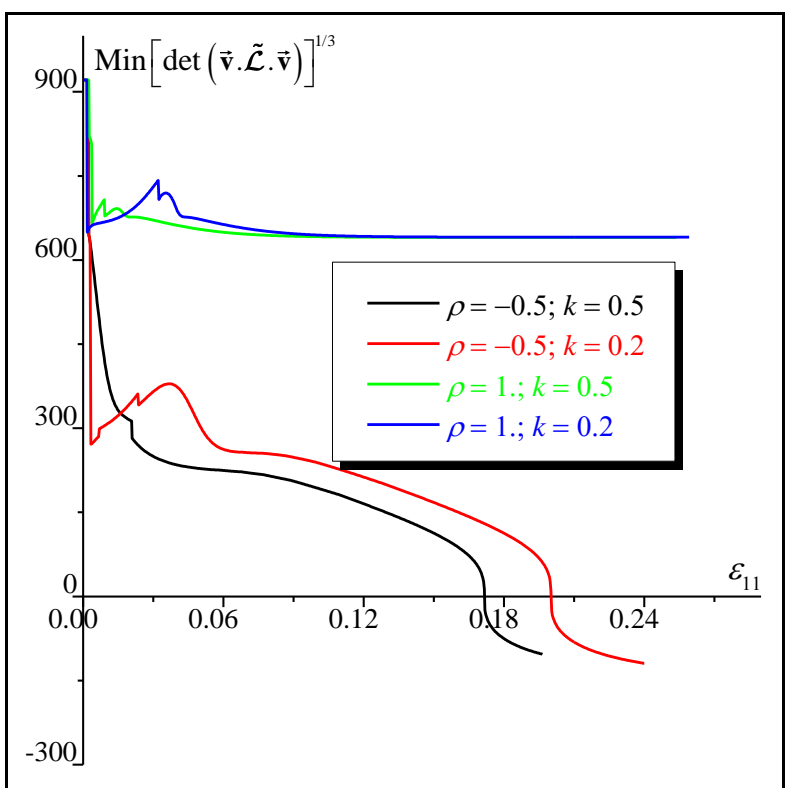

(c)

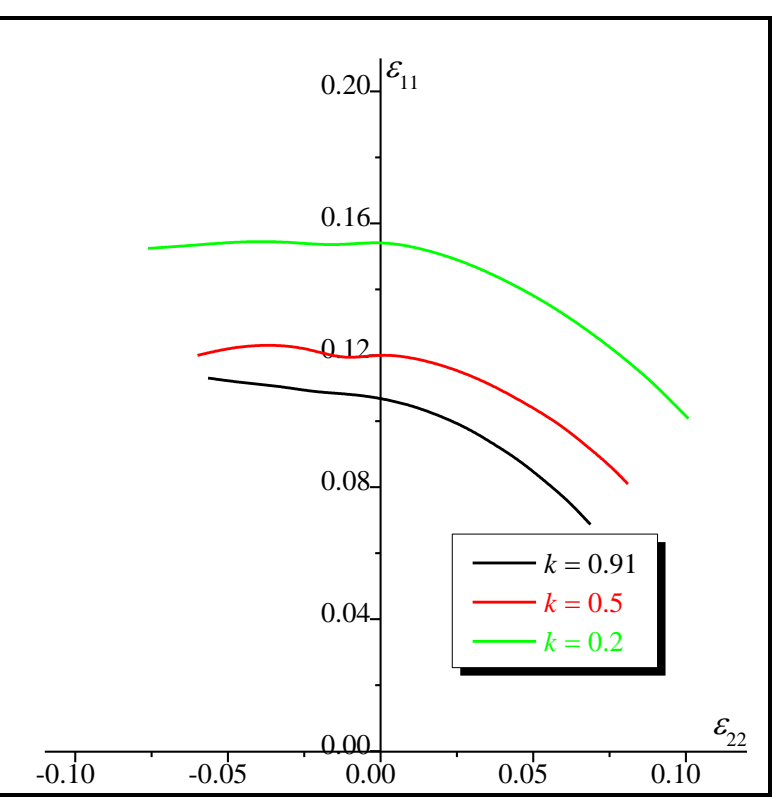

(b)

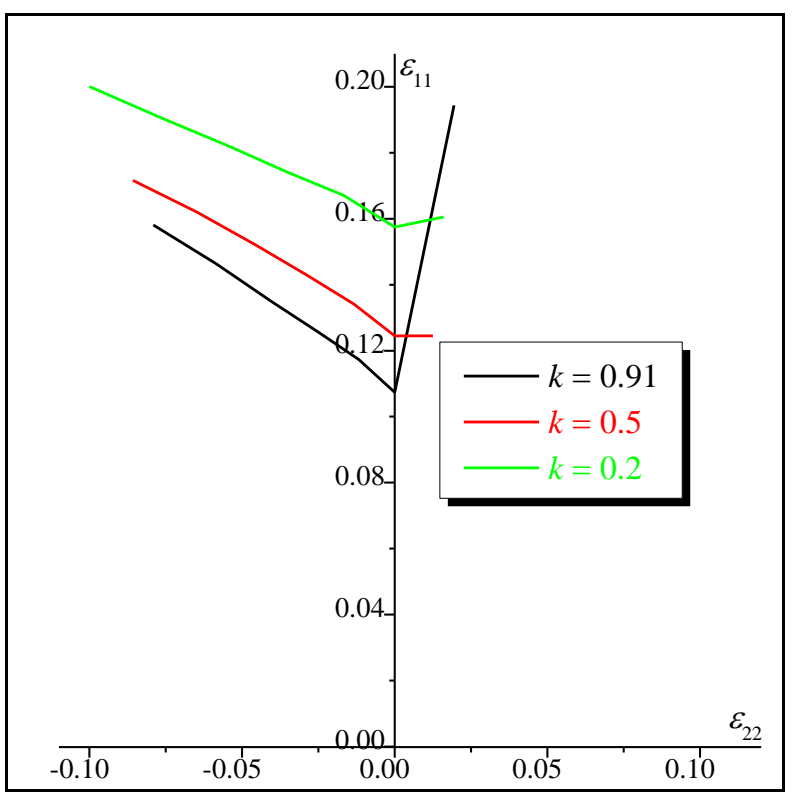

(d) 


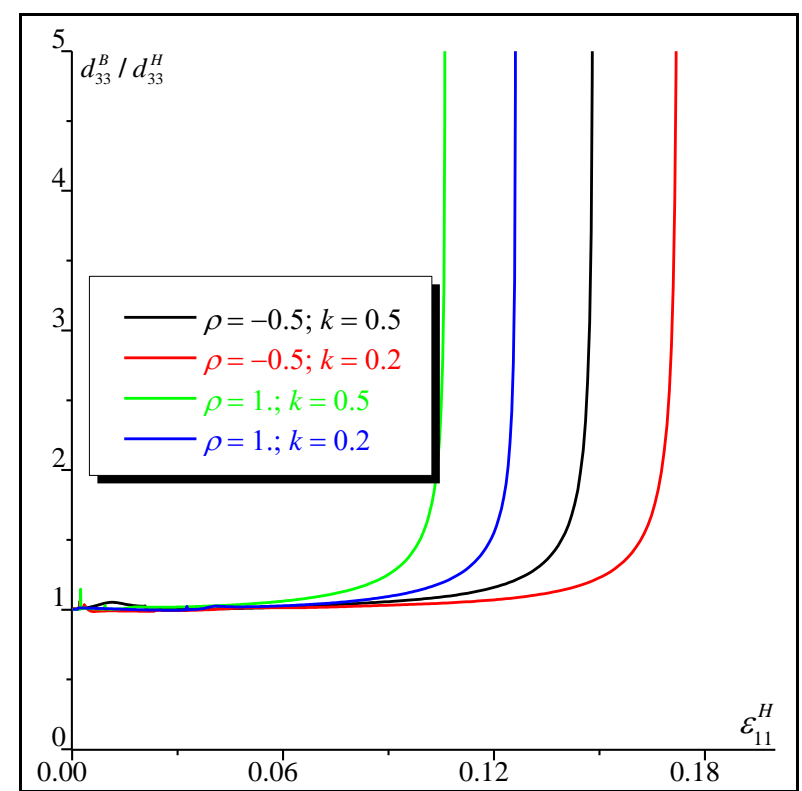

(e)

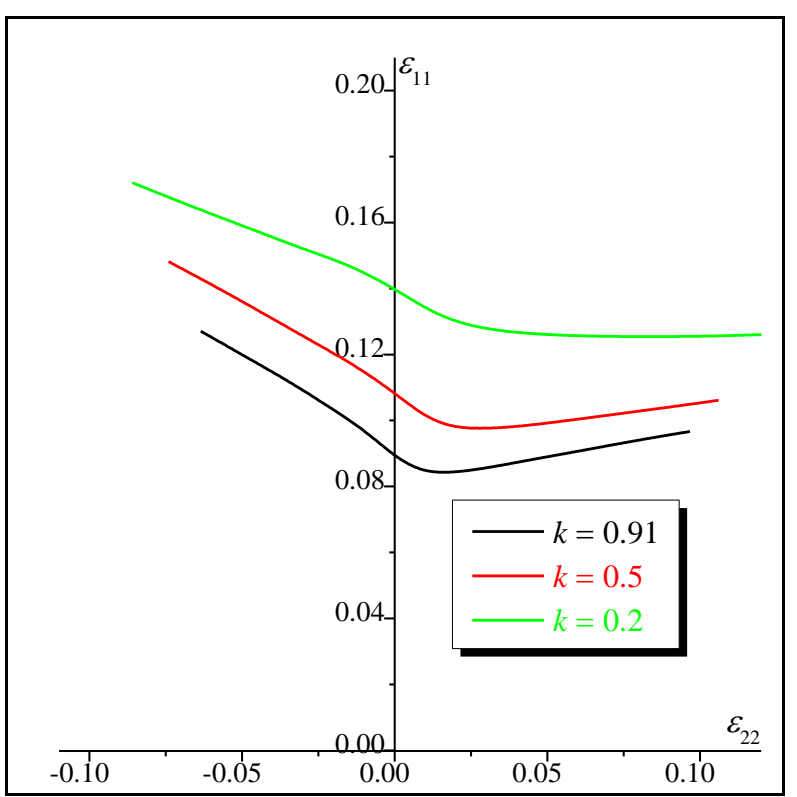

(f)

Fig. 9. Effect of the asymmetry parameter $k$ on: (a) the evolution of the cubic root of the determinant of $\tilde{\mathcal{L}}_{\text {sym }}^{\text {p }}$ versus $\varepsilon_{11}$ (general bifurcation criterion); (b) FLDs predicted by the general bifurcation criterion; (c) the evolution of the minimum of the cubic root of the determinant of the acoustic tensor $\operatorname{det}(\overrightarrow{\mathbf{v}} \cdot \tilde{\mathcal{L}} \cdot \overrightarrow{\mathbf{v}})$ versus $\varepsilon_{11}$ (Rice bifurcation analysis); (d) FLDs predicted by the Rice bifurcation analysis; (e) the evolution of the ratio $d_{33}^{B} / d_{33}^{H}$ versus $\varepsilon_{11}^{H}(\mathrm{M}-\mathrm{K}$ approach); (f) FLDs predicted by the $\mathrm{M}-\mathrm{K}$ approach.

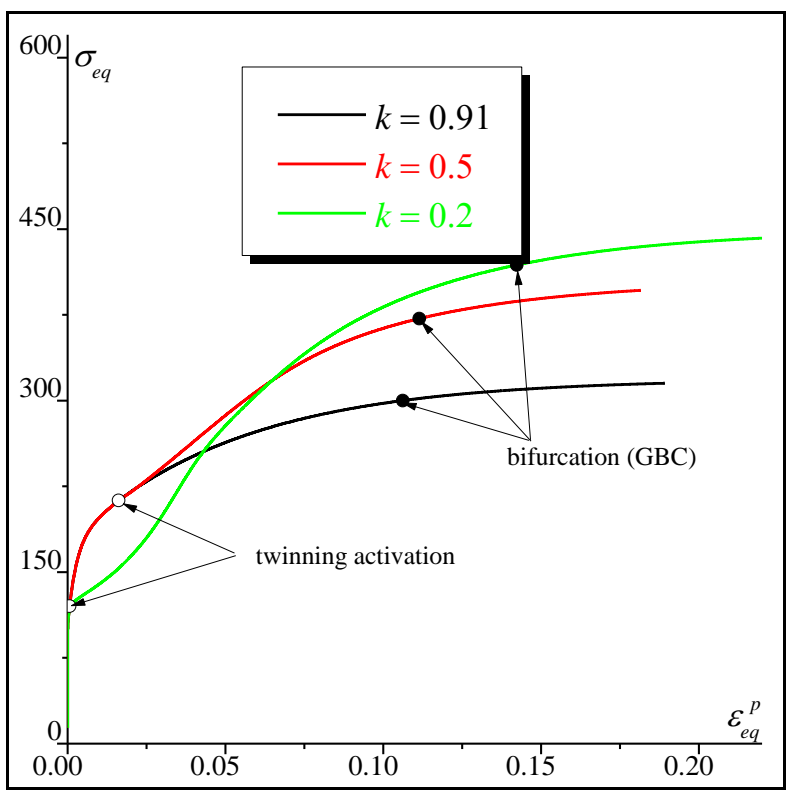

(a)

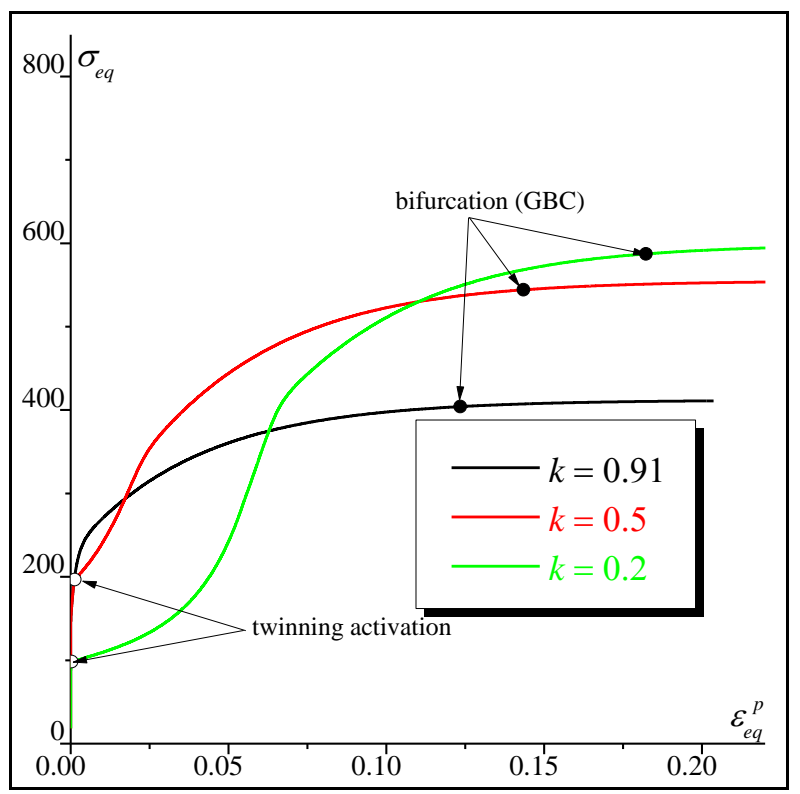

(b) 


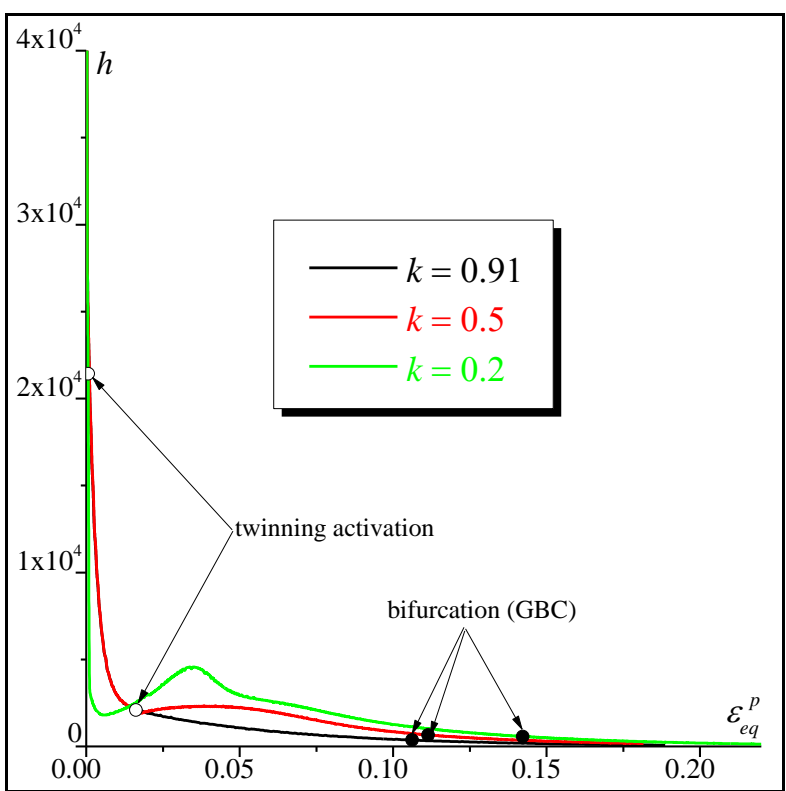

(c)

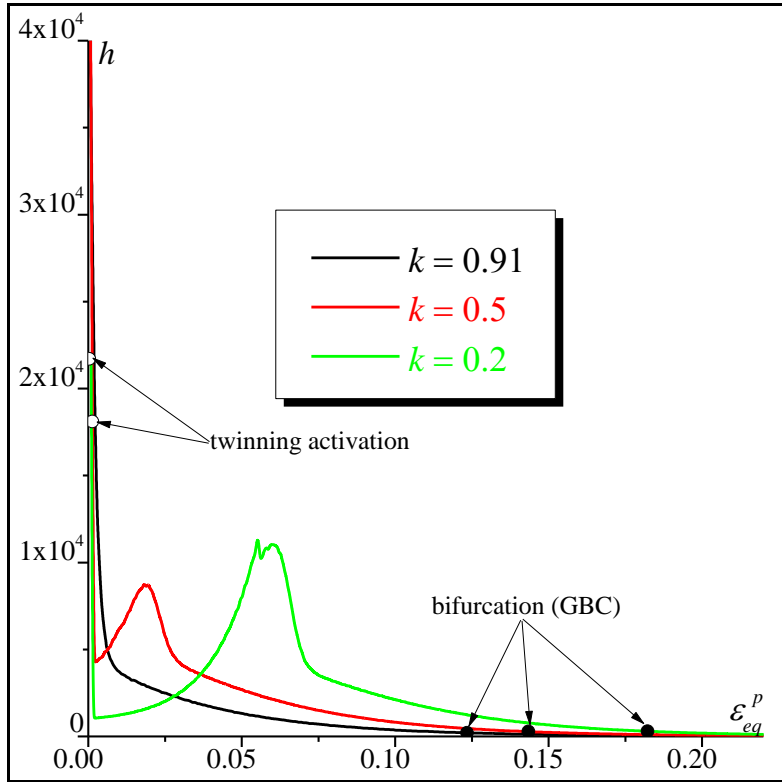

(d)

Fig. 10. Effect of the asymmetry parameter $k$ on: (a) the evolution of $\sigma_{e q}$ versus $\varepsilon_{e q}^{p}$ (uniaxial tension state); (b) the evolution of $\sigma_{e q}$ versus $\varepsilon_{e q}^{p}$ (equibiaxial tension state); (c) the evolution of the hardening modulus $h$ versus $\varepsilon_{e q}^{p}$ (uniaxial tension state); (d) the evolution of the hardening modulus $h$ versus $\varepsilon_{e q}^{p}$ (equibiaxial tension state).

The linear transformation, represented by the fourth-order tensor $\mathbf{L}^{s}$, has been initially introduced in Barlat (1991) to better capture the rotation and the distortion of the slip yield surface. To analyze the effect of this linear transformation on the predicted limit strains, two different sets of coefficients of matrix $\mathbf{L}^{s}$, as reported in Table 2, are used in the simulations shown in Fig. 11. Set 1 in this table is the same as the one given in Table 1. However, the second set (Set 2) is chosen in such a way that the transformation matrix $\mathbf{L}^{s}$ reduces to the identity tensor (and hence the associated yield surface becomes isotropic). The other material parameters are the same as those given in Table 1. The effect of the different coefficient sets on the shape of the initial slip yield surface is depicted in Fig. 11a. Similar to some studies in the literature (see, e.g., Wu et al., 2004), these yield surfaces are normalized by their corresponding equibiaxial yield stresses $\left(\sigma_{11}=\sigma_{22}\right)$, in order to underline their differences in terms of shape of the yield surface. Due to the large value of the strength differential parameter $k=0.91$, only the slip mode is activated for the various strain paths investigated. Consequently, the differences between the FLDs predicted in Fig. 11b and c are uniquely due to the distortion degree of the slip yield surface. The results of Fig. $11 \mathrm{~b}$ reveal that the effect of the choice of the coefficients of matrix $\mathbf{L}^{s}$ on the prediction of the onset of diffuse necking is very slight especially for the positive strain-path ratios. On the other hand, the shape and the level of forming limit diagrams predicted by the initial imperfection approach are more affected by the choice of the components of the linear transformation matrix $\mathbf{L}^{s}$, as demonstrated in Fig. 11c. Indeed, the level of localized necking limit strains can be correlated to the shape of the yield locus: the sharper yield locus corresponds to the 
lower localization limit strain (Barlat, 1987; Akpama et al., 2017). As demonstrated in Fig. 11a, the choice of the components of $\mathbf{L}^{s}$ affects the shape of the yield locus for the whole range of stress ratios. Consequently, this choice influences the level and the shape of the complete forming limit diagrams, as shown in Fig. 11c.

Table 2. Coefficients of the transformation matrix $\mathbf{L}^{s}$.

\begin{tabular}{|l|ccccccccc|}
\hline & $l_{11}^{s}$ & $l_{22}^{s}$ & $l_{33}^{s}$ & $l_{12}^{s}$ & $l_{23}^{s}$ & $l_{13}^{s}$ & $l_{44}^{s}$ & $l_{55}^{s}$ & $l_{66}^{s}$ \\
\hline Set 1 & 1.06 & 1.58 & 0.898 & 1.41 & 1 & 1 & 1 & 1 & 1 \\
\hline Set 2 & 1 & 1 & 1 & 0 & 0 & 0 & 1 & 1 & 1 \\
\hline
\end{tabular}

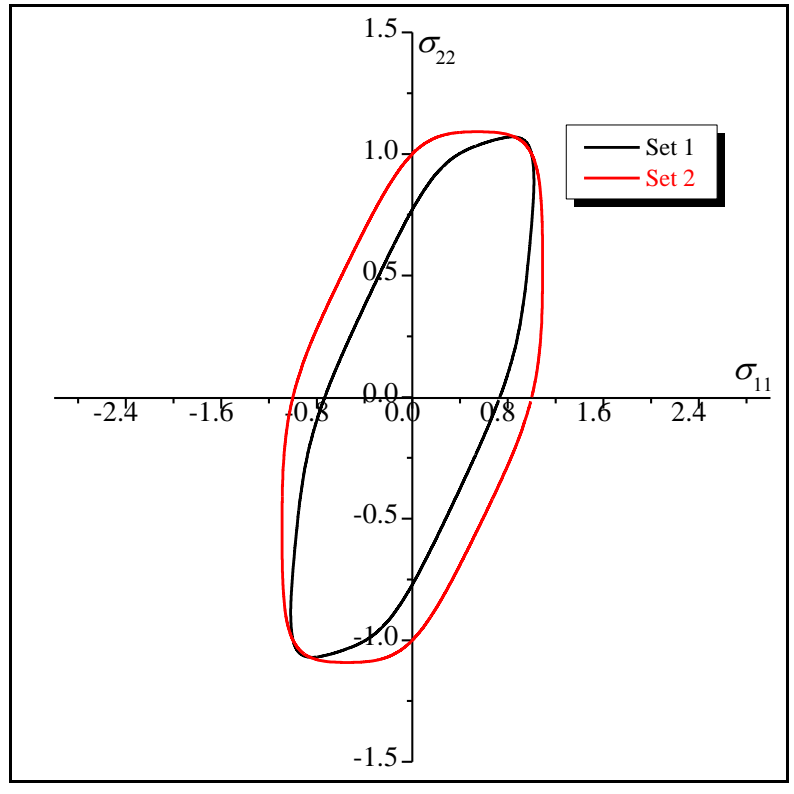

(a)

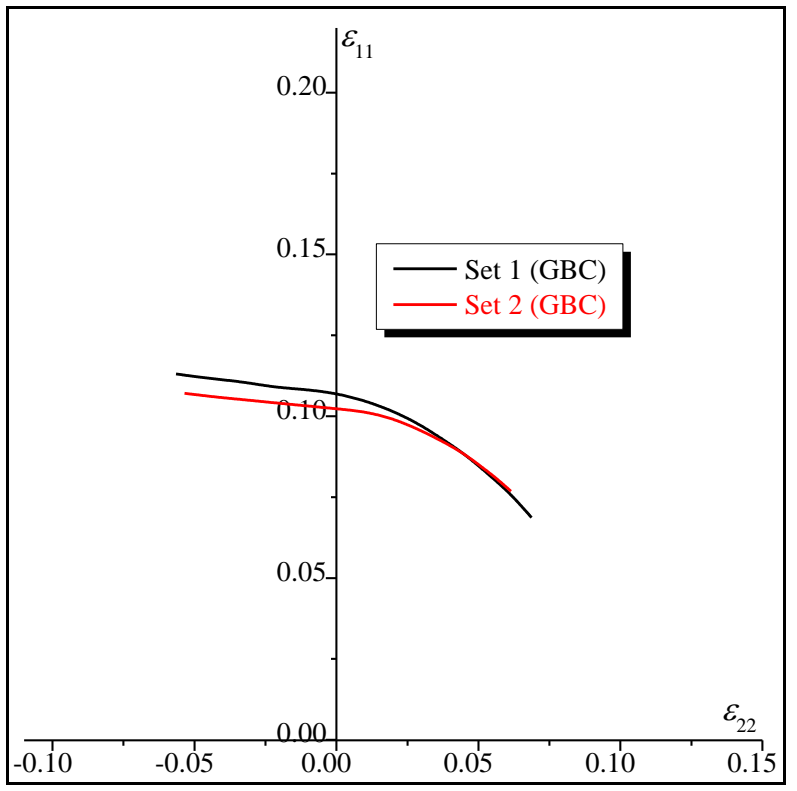

(b)

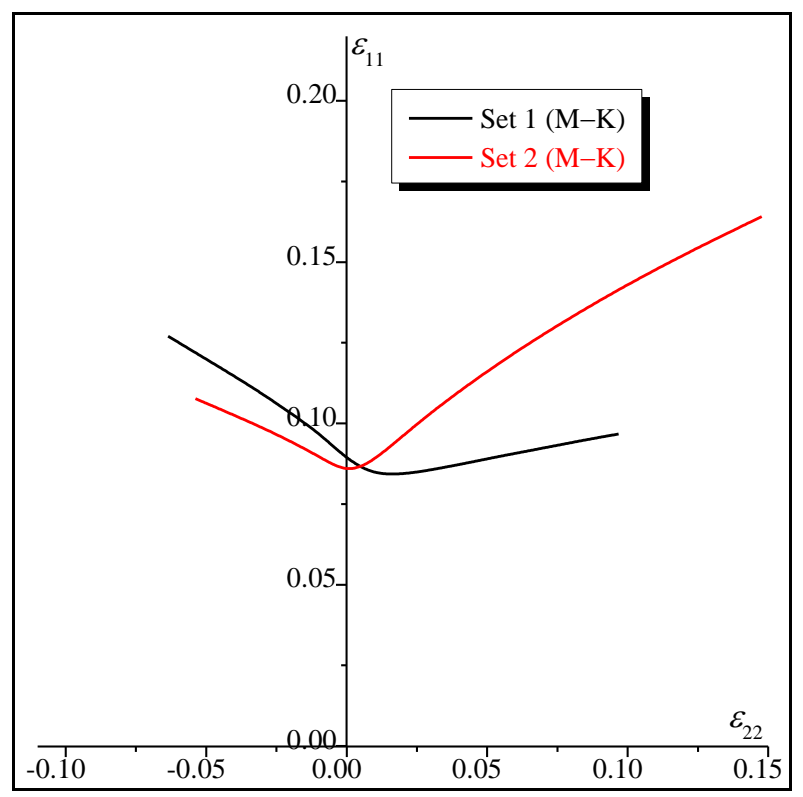

(c) 
Fig. 11. Effect of the components of the linear transformation matrix of the slip yield function on: (a) the shape of the initial yield surface; (b) the forming limit diagrams predicted by the general bifurcation criterion; (c) the forming limit diagrams predicted by the initial imperfection approach.

In the following part, focus will be confined to the study of the effect of the slip anisotropy exponent $a^{s}$ on the occurrence of plastic instability. For this task, two values for exponent $a^{s}$ are used in the predictions shown in Fig. 12: 7.81 (the same as the value given in Table 1) and 4.0. The other parameters are the same as those given in Table 1. Similar to the case of the predictions reported in Fig. 11, twinning deformation mode is never activated for the different simulations shown in Fig. 12. Unlike the effect of the linear transformation coefficients, the effect of coefficient $a^{s}$ on the shape of the initial yield surface is very small for most stress ratios, and only significant for stress ratios near the equibiaxial stress state, as shown in Fig. 12a. Consequently, the necking limit strains predicted by the $\mathrm{M}-\mathrm{K}$ approach are significantly affected by the value of $a^{s}$ for strain paths near the equibiaxial tension state, as demonstrated in Fig. 12c. However, strain localization is almost unaffected by the value of this coefficient for the range of negative strain-path ratios. On the other hand, the predictions of diffuse necking are almost insensitive to exponent $a^{s}$, on the whole, and more particularly in the range of positive strain-path ratios.

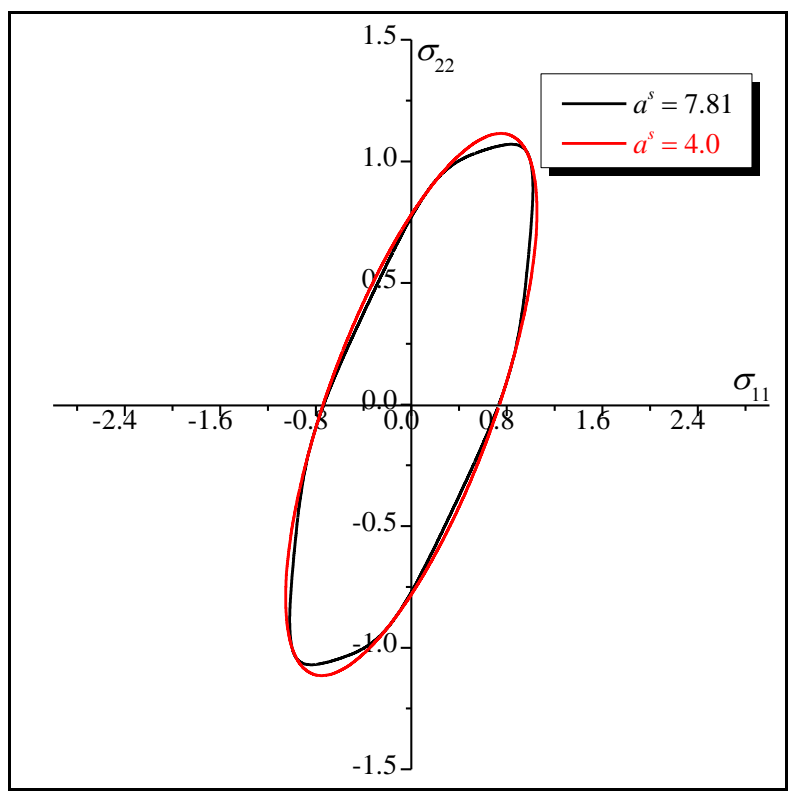

(a)

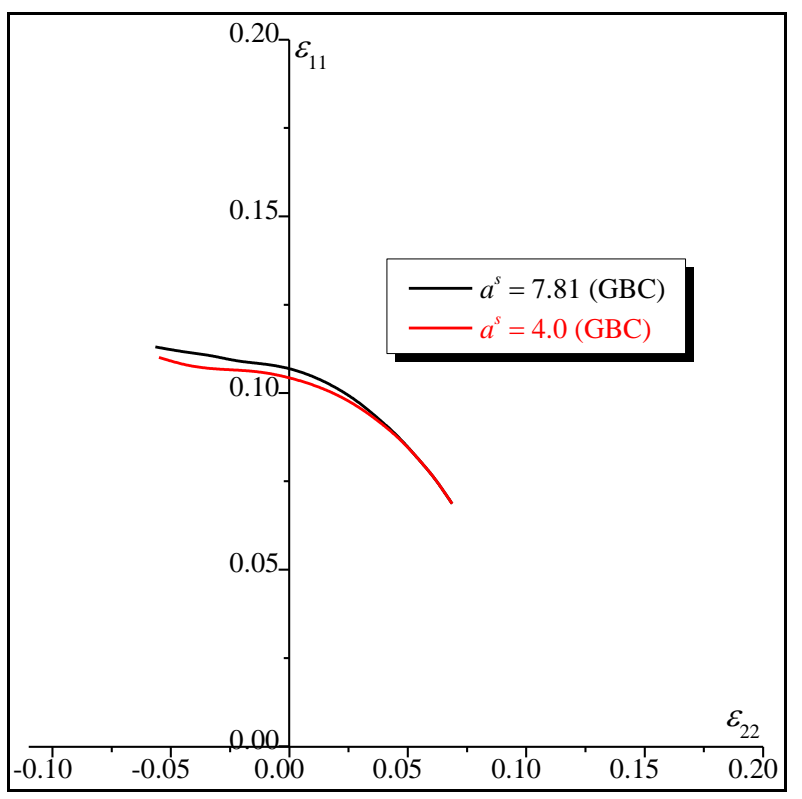

(b) 


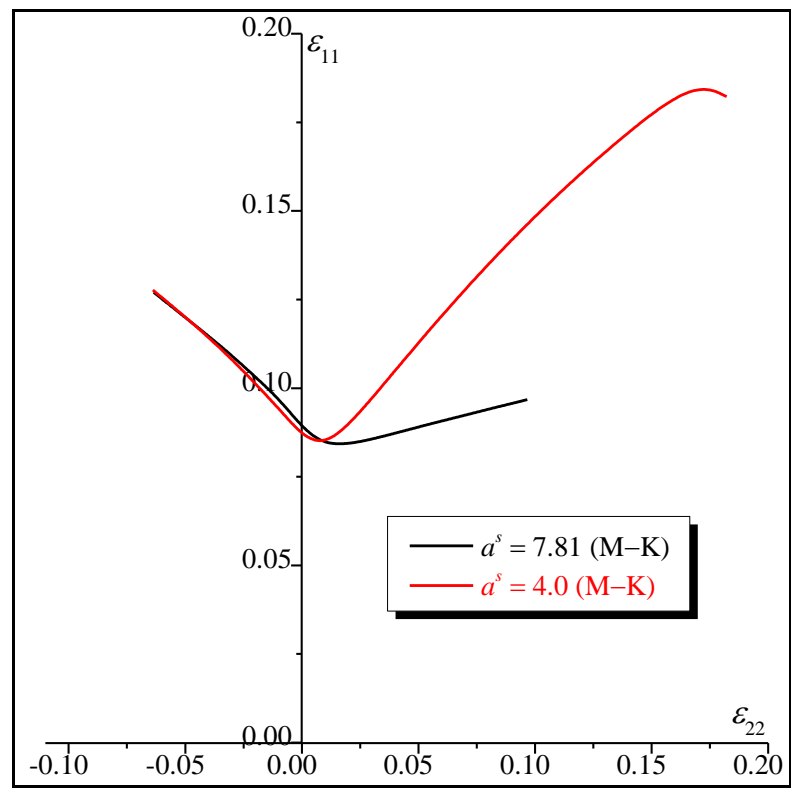

(c)

Fig. 12. Effect of the slip anisotropy exponent $a^{s}$ on: (a) the shape of the initial yield surface; (b) the forming limit diagrams predicted by the general bifurcation criterion; (c) the forming limit diagrams predicted by the initial imperfection approach.

\subsection{Effect of distortional hardening on the prediction of FLDs}

It has been experimentally observed that crystallographic texture in HCP materials rapidly evolves during plastic deformation (Yoon et al., 2013; Ghaffari Tari et al., 2014; Muhammad et al., 2015; Lee et al., 2017). This rapid evolution is mainly due to the activation of the twinning deformation mode. To account for this rapid change, Yoon et al. (2013) assumed that the anisotropy parameters evolve during loading, as a function of the accumulated plastic strain $\gamma$. The experimental results presented in Yoon et al. (2013) are used in the current investigation, as input for the developed numerical tools, for the prediction of the ductility limits of AZ31 magnesium alloy. In contrast to the model formulated in Steglich et al. (2016) and used in the first part of the simulations, plastic anisotropy and its evolution are modeled by the Cazacu yield function only (see Eq. (12)) in Yoon et al. (2013). In the above investigation, isotropic hardening is defined by the following evolution law for $R^{t}$ :

$$
R^{t}\left(\gamma^{t}\right)=A^{t}-B^{t} \exp \left(-C^{t} \gamma^{t}\right)
$$

The hardening parameters $A^{t}, B^{t}$ and $C^{t}$ have been fitted in Yoon et al. (2013) on the basis of uniaxial tensile tests:

$$
A^{t}=300.44 \mathrm{MPa}, \quad B^{t}=134.23 \mathrm{MPa}, \quad C^{t}=17.4825 .
$$

The evolution of the anisotropic yield function is displayed in Fig. 13a. This figure is the same as the one published in Yoon et al. (2013). The theoretical yield surfaces for magnesium AZ31 alloy according to the Cazacu anisotropic model are plotted for different accumulated strain levels $(0,0.03$, 
0.06, 0.10 and 0.13 ) with solid lines. Note that in this figure, the experimental points used to identify the parameters corresponding to the yield loci are shown by small squares. To clearly highlight the distortion of the yield surface resulting from the introduction of distortional hardening, the yield loci are normalized by the current equibiaxial stress (designated as $\sigma_{\text {Equib. }}$ ) and plotted in Fig. 13b.

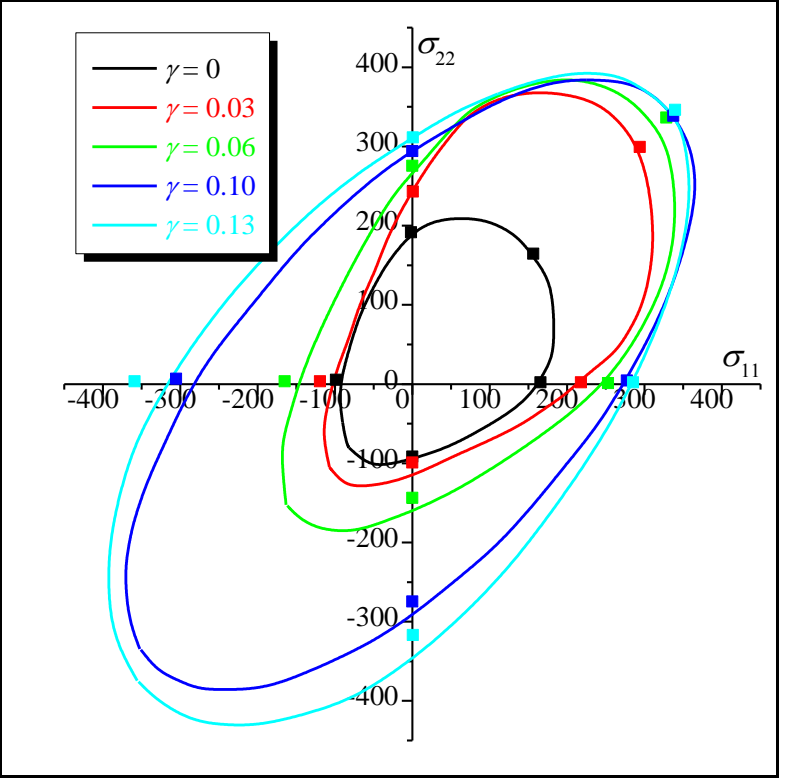

(a)

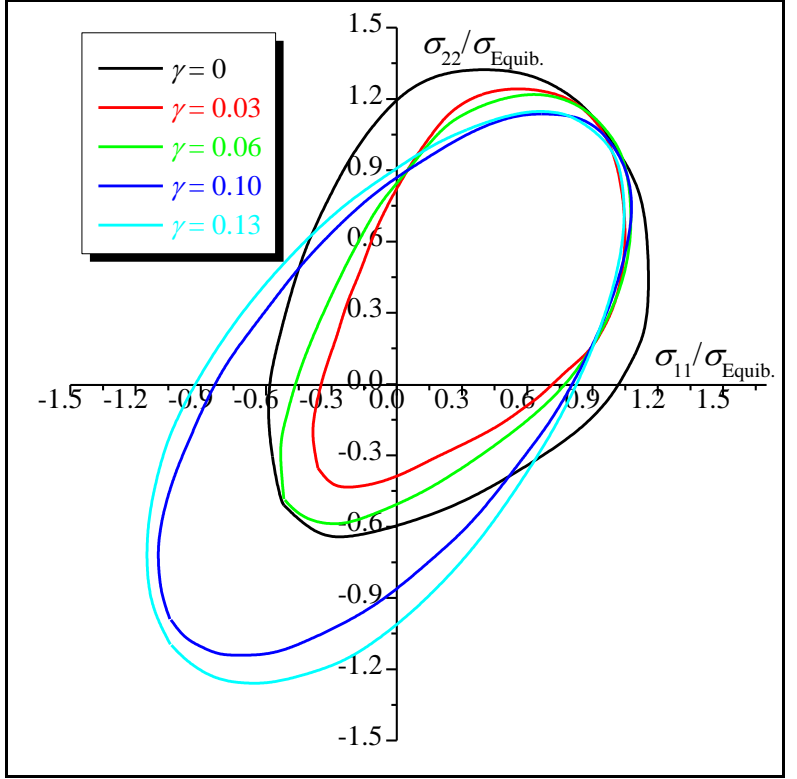

(b)

Fig. 13. Yield loci of AZ31 magnesium sheet corresponding to fixed levels of accumulated twinning: (a) without normalization by the equibiaxial stress; (b) with normalization by the equibiaxial stress.

As five deformation levels are used to determine the evolution of the yield locus, the different anisotropy parameters (the components of the transformation matrix $\mathbf{L}^{t}$ and the strength differential parameter $k$ ) are approximated by polynomials of degree four:

$$
f(\gamma)=\alpha_{0}+\alpha_{1} \gamma+\alpha_{2} \gamma^{2}+\alpha_{3} \gamma^{3}+\alpha_{4} \gamma^{4} .
$$

The values of coefficients $\alpha_{0}, \ldots, \alpha_{4}$ for these polynomial approximations are provided in Table 3 for parameters $l_{22}^{t}, l_{33}^{t}, l_{66}^{t}, l_{12}^{t}, l_{13}^{t}, l_{23}^{t}$ and $k$. The other parameters $\left(l_{11}^{t}, l_{44}^{t}\right.$ and $\left.l_{55}^{t}\right)$ are assumed to be constant during loading and taken equal to 1 . 
Table 3. Numerical values of coefficients $\alpha_{0}, \ldots, \alpha_{5}$ required for the approximation of the different anisotropy parameters.

\begin{tabular}{|c|c|c|c|c|c|}
\hline & $\alpha_{0}$ & $\alpha_{1}$ & $\alpha_{2}$ & $\alpha_{3}$ & $\alpha_{4}$ \\
\hline$l_{22}^{t}$ & 0.9813 & -3.5522, & 1.343 & 878.43 & -5097.8 \\
\hline$l_{33}^{t}$ & -0.0043 & -4.4209 & 197.12 & -3155.1 & 16113 \\
\hline$l_{66}^{t}$ & 0.7427 & 1.917 & -75.573 & 1221.7 & -4667.5 \\
\hline$l_{12}^{t}$ & 0.2795 & -6.2145 & 134.51 & -1415 & 4861.7 \\
\hline$l_{13}^{t}$ & -0.1525 & 0.812 & 55.506 & -1091.9 & 4687.4 \\
\hline$l_{23}^{t}$ & -0.0574 & 1.6746 & -38.611 & 285.94 & -679.03 \\
\hline$k$ & 0.3331 & 10.446 & -214.09 & 505.25 & 2526.9 \\
\hline
\end{tabular}

The theoretical evolution of some anisotropy parameters with plastic strain according to Eq. (69) is represented by solid lines in Fig. 14. This figure confirms that the theoretical curves fit well the experimental points (represented by small squares).

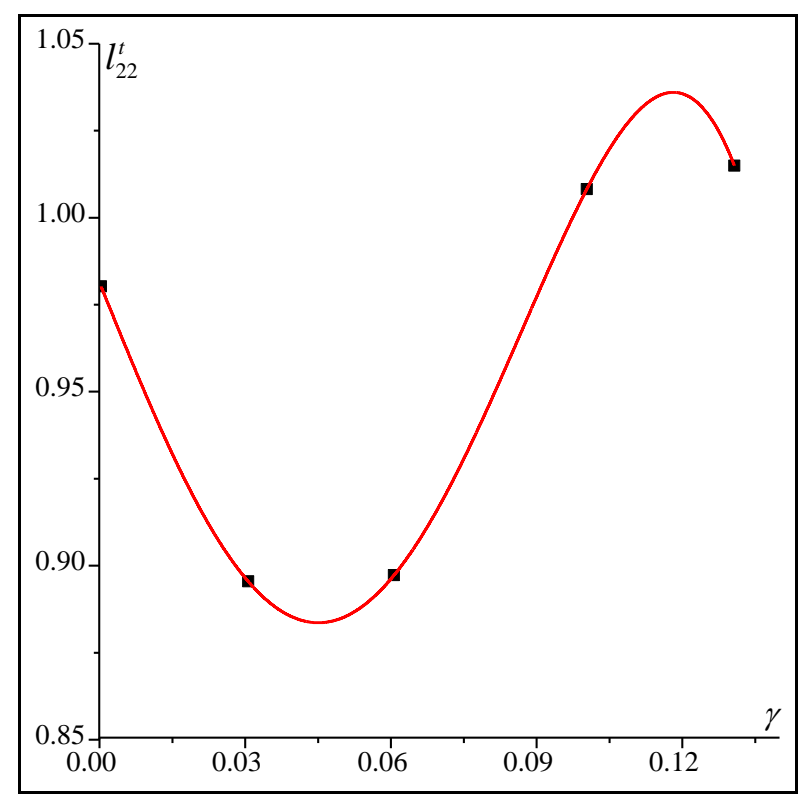

(a)

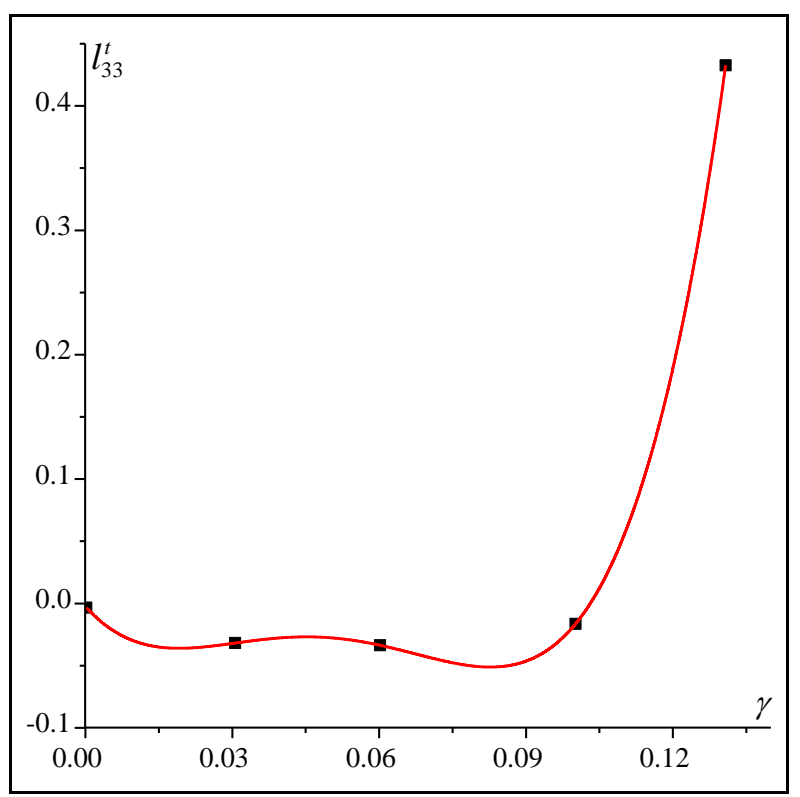

(b) 


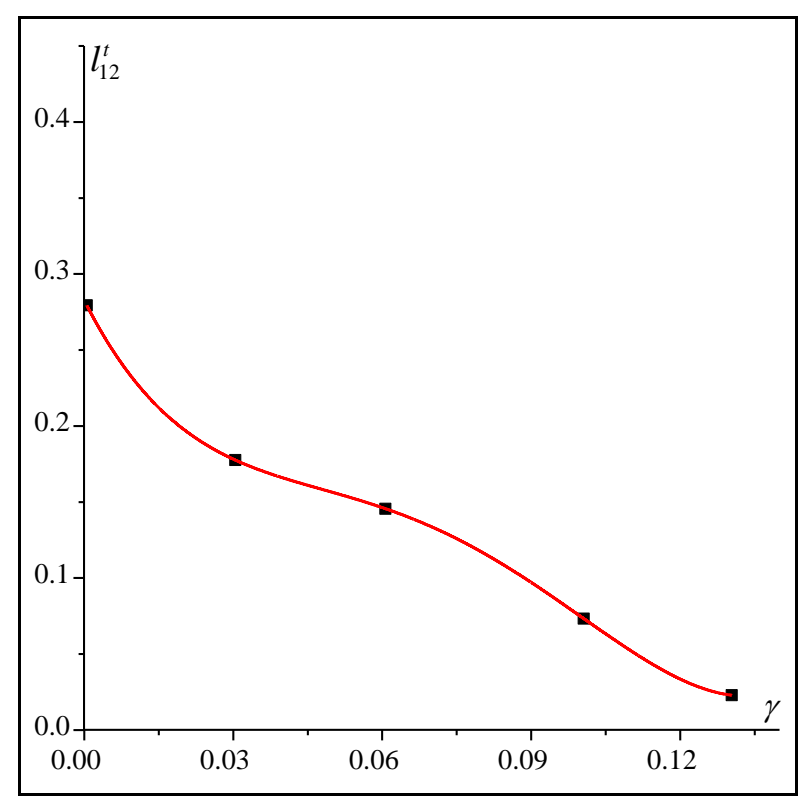

(c)

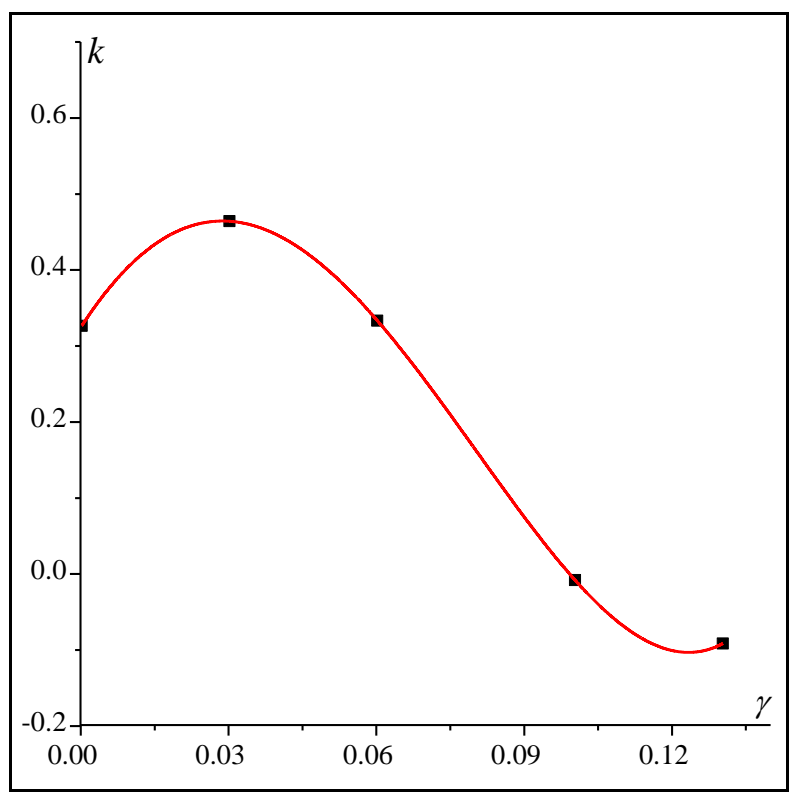

(d)

Fig. 14. Evolution of the anisotropy parameters with plastic strain according to Eq. (69): (a) $l_{22}^{t}$; (b) $l_{33}^{t}$; (c) $l_{12}^{t}$;

(d) $k$.

The anisotropy parameters identified in the above developments have been used as input for the numerical tool based on the initial imperfection approach for the prediction of the ductility limits of the magnesium AZ31 alloy. The effect of the consideration of distortional hardening on the shape and the level of the forming limit diagrams is analyzed through the curves plotted in Fig. 15. In this figure, the initial imperfection factor $f_{0}$ is set to $5 \times 10^{-3}$ and, for the case without distortional hardening, the anisotropy parameters are assumed to be constant and equal to their initial values. The effect of distortional hardening is more pronounced in the range of positive strain-path ratios, as shown in Fig. 15. This result is expectable considering the fact that distortional hardening significantly influences the evolution of the yield surface. Similar to the predictions of Figs. 11 and 12, the level of the predicted ductility limits near the equibiaxial tensile state may likely be correlated with the shape of the yield surfaces plotted in Fig. 13. Furthermore, when the anisotropy parameters are assumed to be constant, remaining equal to their initial values (i.e., the effect of distortional hardening is disregarded), the limit strains predicted in the range of positive strain-path ratios (especially for strain-path ratios $\rho>0.5$ ) are excessively high. This result is not very consistent with the experimental observations showing the low ductility of HCP materials. Consequently, it is essential to incorporate the distortional hardening in the constitutive modeling in order to obtain more realistic results. 


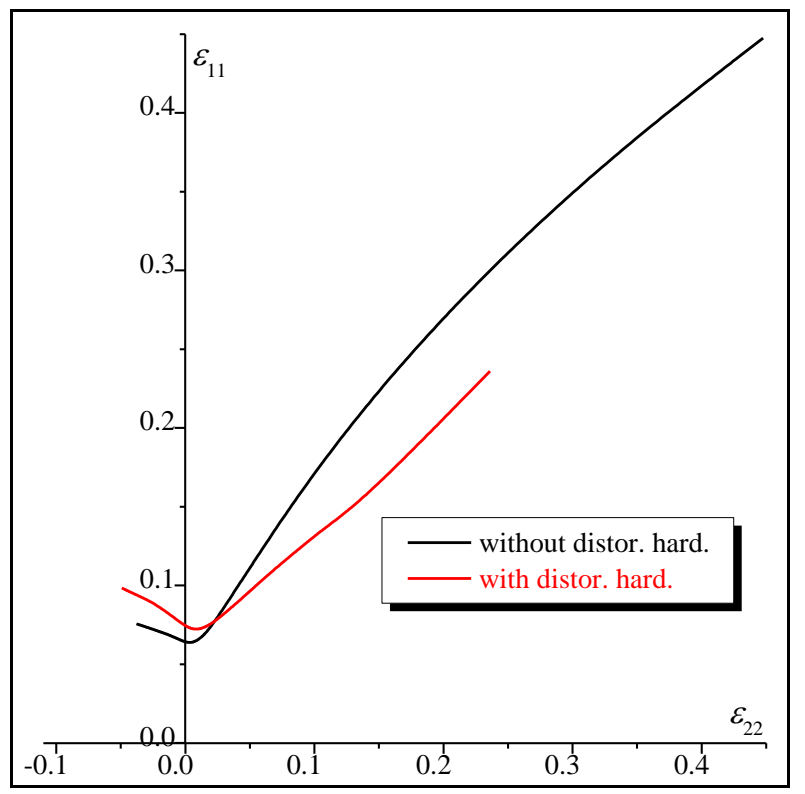

Fig. 15. Effect of distortional hardening on the FLDs predicted by the initial imperfection approach.

\section{Concluding remarks}

In this paper, a two-surface plasticity constitutive framework has been used to model the mechanical behavior of HCP materials taking into account both twinning and slip deformation modes. This model allows incorporating the effect on the mechanical response of distortional hardening resulting from the rapid change of crystallographic texture. Particular attention has been paid to the determination of the continuum tangent modulus and to the implicit integration of the corresponding constitutive equations. The accuracy and the efficiency of the developed numerical algorithm have been assessed on the basis of some numerical predictions. This algorithm can be easily implemented into a finite element code for the simulation of complex sheet metal forming applications involving HCP materials. This integration algorithm is used in the present contribution, in conjunction with three plastic instability criteria (namely the general bifurcation criterion, the Rice bifurcation theory and the initial imperfection approach), to predict the onset of diffuse and localized necking in HCP materials. Results of the numerical predictions are summarized as follows:

- When the material parameters of the AZ31 magnesium alloy are used (which are identified in Steglich et al. (2016) based on experimental data), only the slip deformation mode is activated for the whole range of strain paths involved in the determination of forming limit diagrams.

- For the plane-strain tension state, the simulation results reveal that diffuse necking predicted by the general bifurcation criterion and localized necking determined by the Rice bifurcation approach occur almost simultaneously. 
- The shape and the level of the predicted forming limit diagrams are strongly sensitive to the strength differential parameter $k$. Indeed, small values for this parameter will promote the activation of the twinning mode, whereas large values will delay it.

- When the size of the initial imperfection tends towards zero, the corresponding FLDs tend towards the Rice bifurcation-based FLD.

- The level of limit strains predicted by the initial imperfection approach is mainly dependent on the shape of the yield locus, which depends in turn on the plastic anisotropy parameters. By contrast, the predictions of diffuse necking are only slightly affected by plastic anisotropy.

- The effect of the consideration of distortional hardening on the prediction of the ductility limits is very important, especially in the range of positive strain-path ratios. Therefore, for the accurate validation of the developed numerical tools, with respect to experimental results, it is essential to incorporate distortional hardening in the constitutive modeling.

- The present study has been purposely limited to the prediction of plastic instability using a twosurface plasticity approach. The implementation of the proposed implicit integration algorithm into a finite element code for the modeling of complex sheet metal forming applications will be the subject of future investigations.

\section{Appendix A}

The objective of this appendix is to determine the expressions of $\partial \bar{\sigma}^{s} / \partial \boldsymbol{\sigma}, \partial \bar{\sigma}^{t} / \partial \boldsymbol{\sigma}, M^{s s}, M^{s t}$, $M^{t s}$ and $M^{t t}$. The derivatives $\partial \bar{\sigma}^{s} / \partial \sigma_{i j}$ and $\partial \hat{\sigma}^{t} / \partial \sigma_{i j}(i, j=1,2)$ can be expressed as follows:

$$
\forall i, j=1,2: \quad \frac{\partial \hat{\sigma}^{s}}{\partial \sigma_{i j}}=\frac{\partial \hat{\sigma}^{s}}{\partial \Sigma_{h}^{s}} \frac{\partial \Sigma_{h}^{s}}{\partial \sum_{k l}^{s}} \frac{\partial \sum_{k l}^{s}}{\partial \sigma_{i j}}, \quad \frac{\partial \hat{\sigma}^{t}}{\partial \sigma_{i j}}=\frac{\partial \hat{\sigma}^{t}}{\partial \Sigma_{h}^{t}} \frac{\partial \Sigma_{h}^{t}}{\partial \Sigma_{k l}^{t}} \frac{\partial \Sigma_{k l}^{t}}{\partial \sigma_{i j}}, \quad h, k, l=1,2,3 .
$$

To determine $\partial \hat{\sigma}^{s} / \partial \sigma_{i j}$ and $\partial \hat{\sigma}^{t} / \partial \sigma_{i j}$, the analytical expressions of $\partial \hat{\sigma}^{s} / \partial \Sigma_{h}^{s}$ and $\partial \hat{\sigma}^{t} / \partial \Sigma_{h}^{t}$ should be first computed:

$$
\begin{aligned}
& \frac{\partial \hat{\sigma}^{s}}{\partial \Sigma_{1}^{s}}=\frac{\left(\Sigma_{1}^{s}-\Sigma_{2}^{s}\right)^{a^{s}-1}+\left(\Sigma_{1}^{s}-\Sigma_{3}^{s}\right)^{a^{s}-1}}{\left(\left|\Sigma_{1}^{s}-\Sigma_{2}^{s}\right|^{s^{s}}+\left|\Sigma_{2}^{s}-\Sigma_{3}^{s}\right|^{a^{s}}+\left|\Sigma_{1}^{s}-\Sigma_{3}^{s}\right|^{a^{s}}\right)} \hat{\sigma}^{s}, \\
& \frac{\partial \hat{\sigma}^{s}}{\partial \Sigma_{2}^{s}}=\frac{\left(\Sigma_{2}^{s}-\Sigma_{3}^{s}\right)^{a^{s}-1}-\left(\Sigma_{1}^{s}-\Sigma_{2}^{s}\right)^{a^{s}-1}}{\left(\left|\Sigma_{1}^{s}-\Sigma_{2}^{s}\right|^{a^{s}}+\left|\Sigma_{2}^{s}-\Sigma_{3}^{s}\right|^{a^{s}}+\left|\Sigma_{1}^{s}-\Sigma_{3}^{s}\right|^{a^{s}}\right)} \widehat{\sigma}^{s}, \\
& \frac{\partial \hat{\sigma}^{s}}{\partial \Sigma_{3}^{s}}=-\frac{\left(\Sigma_{2}^{s}-\Sigma_{3}^{s}\right)^{a^{s}-1}+\left(\Sigma_{1}^{s}-\Sigma_{3}^{s}\right)^{a^{s}-1}}{\left(\left|\Sigma_{1}^{s}-\Sigma_{2}^{s}\right|^{a^{s}}+\left|\Sigma_{2}^{s}-\Sigma_{3}^{s}\right|^{a^{s}}+\left|\Sigma_{1}^{s}-\Sigma_{3}^{s}\right|^{a^{s}}\right)} \hat{\sigma}^{s}, \\
& \forall h=1,2,3: \quad \frac{\partial \hat{\sigma}^{t}}{\partial \Sigma_{h}^{t}}=\frac{\left(\left|\Sigma_{h}^{t}\right|-k \Sigma_{h}^{t}\right)^{a^{t}-1}\left(\operatorname{sgn}\left(\Sigma_{h}^{t}\right)-k\right)}{\left(\left(\left|\Sigma_{1}^{t}\right|-k \Sigma_{1}^{t}\right)^{a^{t}}+\left(\left|\Sigma_{2}^{t}\right|-k \Sigma_{2}^{t}\right)^{a^{t}}+\left(\left|\Sigma_{3}^{t}\right|-k \Sigma_{3}^{t}\right)^{a^{t}}\right)} \hat{\sigma}^{t} .
\end{aligned}
$$


Under the plane-stress conditions, tensor $\Sigma^{*}$ (where $\left.*=s, t\right)$ can be generically expressed in the following form:

$$
\Sigma^{*}=\left[\begin{array}{ccc}
\Sigma_{11}^{*} & \Sigma_{12}^{*} & 0 \\
\Sigma_{12}^{*} & \Sigma_{22}^{*} & 0 \\
0 & 0 & \Sigma_{33}^{*}
\end{array}\right] .
$$

Consequently, the derivatives $\frac{\partial \Sigma_{h}^{*}}{\partial \Sigma_{k l}^{*}}$ can be obtained by the following expressions:

$$
\begin{aligned}
& \frac{\partial \Sigma_{1}^{*}}{\partial \Sigma_{11}^{*}}=\frac{1}{2}\left(1+\frac{\Sigma_{11}^{*}-\Sigma_{22}^{*}}{\lambda^{*}}\right), \quad \frac{\partial \Sigma_{1}^{*}}{\partial \Sigma_{22}^{*}}=\frac{1}{2}\left(1+\frac{-\Sigma_{11}^{*}+\Sigma_{22}^{*}}{\lambda^{*}}\right), \quad \frac{\partial \Sigma_{1}^{*}}{\partial \Sigma_{12}^{*}}=\frac{2 \Sigma_{12}^{*}}{\lambda^{*}}, \frac{\partial \Sigma_{1}^{*}}{\partial \Sigma_{33}^{*}}=0, \\
& \frac{\partial \Sigma_{2}^{*}}{\partial \Sigma_{11}^{*}}=\frac{1}{2}\left(1+\frac{-\Sigma_{11}^{*}+\Sigma_{22}^{*}}{\lambda^{*}}\right), \quad \frac{\partial \Sigma_{2}^{*}}{\partial \Sigma_{22}^{*}}=\frac{1}{2}\left(1+\frac{\Sigma_{11}^{*}-\Sigma_{22}^{*}}{\lambda^{*}}\right), \frac{\partial \Sigma_{2}^{*}}{\partial \Sigma_{12}^{*}}=-\frac{2 \Sigma_{12}^{*}}{\lambda^{*}}, \frac{\partial \Sigma_{2}^{*}}{\partial \Sigma_{33}^{*}}=0, \\
& \frac{\partial \Sigma_{3}^{*}}{\partial \Sigma_{11}^{*}}=0, \quad \frac{\partial \Sigma_{3}^{*}}{\partial \Sigma_{22}^{*}}=0, \quad \frac{\partial \Sigma_{3}^{*}}{\partial \Sigma_{12}^{*}}=0, \quad \frac{\partial \Sigma_{3}^{*}}{\partial \Sigma_{33}^{*}}=1,
\end{aligned}
$$

where $\lambda^{*}$ is given by the following expression:

$$
\lambda^{*}=\sqrt{4\left(\Sigma_{12}^{*}\right)^{2}+\left(\Sigma_{11}^{*}-\Sigma_{22}^{*}\right)^{2}} .
$$

The last derivatives $\partial \Sigma_{k l}^{s} / \partial \sigma_{i j}$ and $\partial \Sigma_{k l}^{t} / \partial \sigma_{i j}$ can be easily determined from Eqs. (9) and (13):

$$
\forall i, j=1,2,3 \text { and } \forall k, l=1,2: \frac{\partial \Sigma_{i j}^{s}}{\partial \sigma_{k l}}=\left(\mathbf{L}^{s} . \mathbf{T}\right)_{i j k l}, \quad \frac{\partial \Sigma_{i j}^{t}}{\partial \sigma_{k l}}=\left(\mathbf{L}^{t} . \mathbf{T}\right)_{i j k l} .
$$

For an easy numerical implementation, tensors $\Sigma^{s}, \Sigma^{t}$ and $\sigma$ may be converted into their vector forms in Eq. (A.6).

Eqs. (A.2)-(A.6) are sufficient to completely determine the derivatives $\partial \hat{\sigma}^{s} / \partial \sigma_{i j}$ and $\partial \hat{\sigma}^{t} / \partial \sigma_{i j}$.

The components $M^{s s}, M^{s t}, M^{t s}$ and $M^{t t}$ are defined by the following generic forms (see Eqs. (26) and (27)):

$$
\begin{aligned}
& M^{s s}=\frac{\partial \Phi^{s}}{\partial \gamma^{s}}=\frac{\partial \hat{\sigma}^{s}}{\partial \gamma^{s}}-\frac{\partial R^{s}}{\partial \gamma^{s}}, \quad M^{s t}=\frac{\partial \Phi^{s}}{\partial \gamma^{t}}=\frac{\partial \hat{\sigma}^{s}}{\partial \gamma^{t}}-\frac{\partial R^{s}}{\partial \gamma^{t}}, \\
& M^{t s}=\frac{\partial \Phi^{t}}{\partial \gamma^{s}}=\frac{\partial \hat{\sigma}^{t}}{\partial \gamma^{s}}-\frac{\partial R^{t}}{\partial \gamma^{s}}, \quad M^{t t}=\frac{\partial \Phi^{t}}{\partial \gamma^{t}}=\frac{\partial \widehat{\sigma}^{t}}{\partial \gamma^{t}}-\frac{\partial R^{t}}{\partial \gamma^{t}} .
\end{aligned}
$$

To simplify the subsequent developments required to compute components $M^{s s}, M^{s t}, M^{t s}$ and $M^{t t}$, the matrix forms (10) and (14) of transformation matrices $\mathbf{L}^{s}$ and $\mathbf{L}^{t}$ are used instead of the fourthorder tensor forms.

If the distortional hardening is not considered in the modeling (as it is the case in the model studied in Section 4.1), components $\partial \hat{\sigma}^{s} / \partial \gamma^{s}, \partial \hat{\sigma}^{s} / \partial \gamma^{t}, \partial \hat{\sigma}^{t} / \partial \gamma^{s}$ and $\partial \hat{\sigma}^{t} / \partial \gamma^{t}$ are obviously equal to 
zero. However, when distortional hardening is considered (as it is the case in the model studied in Section 4.5), the above derivatives should be computed as follows:

$$
\begin{aligned}
& \frac{\partial \hat{\sigma}^{s}}{\partial \gamma^{s}}=\frac{\partial \hat{\sigma}^{s}}{\partial \Sigma_{i j}^{s}} \frac{\partial \sum_{i j}^{s}}{\partial L_{k l}^{s}} \frac{\partial L_{k l}^{s}}{\partial \gamma^{s}}, \quad \frac{\partial \hat{\sigma}^{s}}{\partial \gamma^{t}}=\frac{\partial \hat{\sigma}^{s}}{\partial \Sigma_{i j}^{s}} \frac{\partial \sum_{i j}^{s}}{\partial L_{k l}^{s}} \frac{\partial L_{k l}^{s}}{\partial \gamma^{t}}, \\
& \frac{\partial \hat{\sigma}^{t}}{\partial \gamma^{s}}=\frac{\partial \hat{\sigma}^{t}}{\partial \Sigma_{i j}^{t}} \frac{\partial \sum_{i j}^{t}}{\partial L_{k l}^{t}} \frac{\partial L_{k l}^{t}}{\partial \gamma^{s}}+\frac{\partial \hat{\sigma}^{t}}{\partial k} \frac{\partial k}{\partial \gamma^{s}}, \quad \frac{\partial \hat{\sigma}^{t}}{\partial \gamma^{t}}=\frac{\partial \hat{\sigma}^{t}}{\partial \Sigma_{i j}^{t}} \frac{\partial \Sigma_{i j}^{t}}{\partial L_{k l}^{t}} \frac{\partial L_{k l}^{t}}{\partial \gamma^{t}}+\frac{\partial \hat{\sigma}^{t}}{\partial k} \frac{\partial k}{\partial \gamma^{t}}, \quad i, j, k, l=1,2,3 .
\end{aligned}
$$

Tensors $\partial \widehat{\sigma}^{s} / \partial \Sigma_{i j}^{s}$ and $\partial \hat{\sigma}^{t} / \partial \Sigma_{i j}^{t}$ can be determined from the analytical development of the first part of this appendix, while fourth-order tensors $\partial \Sigma_{i j}^{s} / \partial L_{k l}^{s}$ and $\partial \sum_{i j}^{s} / \partial L_{k l}^{s}$ can be easily determined in the same way as Eqs. (A.6) from Eqs. (9) and (13). On the other hand, $\partial \hat{\sigma}^{t} / \partial k$ can be determined from the expression (12) of $\hat{\sigma}^{t}$ :

$$
\frac{\partial \widehat{\sigma}^{t}}{\partial k}=\widehat{\sigma}^{t} \frac{\left[-\Sigma_{1}^{t}\left(\left|\Sigma_{1}^{t}\right|-k \Sigma_{1}^{t}\right)^{-1+a^{t}}-\Sigma_{2}^{t}\left(\left|\Sigma_{2}^{t}\right|-k \Sigma_{2}^{t}\right)^{-1+a^{t}}-\Sigma_{3}^{t}\left(\left|\Sigma_{3}^{t}\right|-k \Sigma_{3}^{t}\right)^{-1+a^{t}}\right]}{\left(\left|\Sigma_{1}^{t}\right|-k \Sigma_{1}^{t}\right)^{a^{t}}+\left(\left|\Sigma_{2}^{t}\right|-k \Sigma_{2}^{t}\right)^{a^{t}}+\left(\left|\Sigma_{3}^{t}\right|-k \Sigma_{3}^{t}\right)^{a^{t}}} .
$$

Derivatives $\partial L_{k l}^{s} / \partial \gamma^{s}, \partial L_{k l}^{t} / \partial \gamma^{s}, \partial L_{k l}^{s} / \partial \gamma^{t}, \partial L_{k l}^{t} / \partial \gamma^{t}, \partial k / \partial \gamma^{s}$ and $\partial k / \partial \gamma^{t}$ can be determined from the evolution laws of the anisotropy parameters (see Section 4.5).

\section{References}

Akpama, H.K., Ben Bettaieb, M., Abed-Meraim, F., 2016. Numerical integration of rate-independent BCC single crystal plasticity models: comparative study of two classes of numerical algorithms. Int. J. Num. Meth. Eng. 108(5), 363-422.

Akpama, H.K., Ben Bettaieb, M., Abed-Meraim, F., 2017. Localized necking predictions based on rateindependent self-consistent polycrystal plasticity: Bifurcation analysis versus imperfection approach. Int. J. Plast. 91, 205-237.

Barlat, F., 1987. Crystallographic texture, anisotropic yield surfaces and forming limits of sheet metals. Mater. Sci. Eng. 91, 55-72.

Barlat, F., Lege, D.J., Brem, J.C., 1991. A six-component yield function for anisotropic materials. Int. J. Plast. 7(7), 693-712.

Ben Bettaieb, M. and Abed-Meraim, F., 2015. Investigation of localized necking in substrate-supported metal layers: Comparison of bifurcation and imperfection analyses. Int. J. Plast. 65, 168-190.

Bouktir Y., Chalal H., Abed-Meraim F., 2018. Prediction of necking in thin sheet metals using an elastic-plastic model coupled with ductile damage and bifurcation criteria. Int. J. Damage Mech. 27(6), 801-839.

Cazacu, O. and Barlat, F., 2004. A criterion for description of anisotropy and yield differential effects in pressure-insensitive metals. Int. J. Plast. 20(11), 2027-2045. 
Cazacu, O., Plunkett, B., Barlat, F., 2006. Orthotropic yield criterion for hexagonal closed packed metals. Int. J. Plast. 22(7), 1171-1194.

Chang, Q.F., Li, D.Y., Peng, Y.H., Zeng, X.Q., 2007. Experimental and numerical study of warm deep drawing of AZ31 magnesium alloy sheet. Int. J. Mach. Tool. Manu. 47, 436-443.

Chen, F.K., Huang, T.B., 2003. Formability of stamping magnesium-alloy AZ31 sheets. J. Mater. Process. Tech. $142,643-647$.

Drucker, D.C., 1950. Some implications of work hardening and ideal plasticity. Q. Appl. Mathematics 7, 411418.

Drucker, D.C. 1956. On uniqueness in the theory of plasticity. Q. Appl. Mathematics 14, 35-42.

Fischer, A., 1992. A special Newton-type optimization method. Optimization 24(3-4), 269-284.

Fischer, A., 1997. Solution of monotone complementarity problems with locally Lipschitzian functions. Math. Program. 76(3), 513-532.

Fan, X.G., Jiang, X.Q., Zeng, X., Shi, Y.G., Gao, P.F., Zhan, M., 2018. Modeling the anisotropy of hot plastic deformation of two-phase titanium alloys with a colony microstructure. Int. J. Plast. 104, 173-195.

Ghaffari Tari, D., Worswick, M.J., Ali, U., Gharghouri, M.A., 2014. Mechanical response of AZ31B magnesium alloy: experimental characterization and material modeling considering proportional loading at room temperature. Int. J. Plast. 55, 247-267.

Gilles, G., Hammami, W., Libertiaux, V., Cazacu, O., Yoon, J.H., Kuwabara, T., Habraken, A.M., Duchêne, L., 2011. Experimental characterization and elasto-plastic modeling of the quasi-static mechanical response of TA-6 V at room temperature. Int. J. Solids. Struct. 48(9), 1277-1289.

Haddag, B., Abed-Meraim F., Balan T., 2009. Strain localization analysis using a large deformation anisotropic elastic-plastic model coupled with damage. Int. J. Plast. 25, 1970-1996.

Hill, R., 1952. On discontinuous plastic states, with special reference to localized necking in thin sheets. J. Mech. Phys. Solids 1(1), 19-30.

Hill, R., 1958. A general theory of uniqueness and stability in elastic-plastic solids. J. Mech. Phys. Solids 6(3), 236-249.

Hosford, W.F. and T.J. Allen., 1973. Twinning and directional slip as a cause for a strength differential effect. Metall. Mater. Trans. B 4(5), 1424-1425.

Hutchinson, J.W., Neale, K.W., Needleman, A., 1978a. Sheet necking-I. Validity of plane stress assumptions of the long-wavelength approximation. In: Koistinen, D.P., Wang, N.M. (Eds.), Mechanics of Sheet Metal Forming. Plenum, 111-126.

Hutchinson, J.W. and Neale, K.W., 1978b. Sheet necking-II. Time-independent behavior. In: Koistinen, D.P., Wang, N.M. (Eds.), Mechanics of Sheet Metal Forming. Plenum, 127-153.

Kalidindi, S.R., 1998. Incorporation of deformation twinning in crystal plasticity models. J. Mech. Phys. Solids 46(2), 267-290. 
Khan, A.S., Yu, S., Liu, H., 2012. Deformation induced anisotropic responses of Ti-6Al-4V alloy Part II: A strain rate and temperature dependent anisotropic yield criterion. Int. J. Plast. 38, 14-26.

Kim, J.H., Kim, D., Lee, Y.S., Lee, M.G., Chung, K., Kim, H.Y., Wagoner, R.H., 2013. A temperaturedependent elasto-plastic constitutive model for magnesium alloy AZ31 sheets. Int. J. Plast. 50, 66-93.

Kim, J., Kim, S.W., Song, W.J., Kang, B.S., 2005. Analytical and numerical approach to prediction of forming limit in tube hydroforming. Int. J. Mech. Sci. 47, 1023-1037.

Kondori, B., Madi, Y., Besson, J., Benzerga, A.A., 2019. Evolution of the 3D plastic anisotropy of HCP metals: Experiments and modeling. Int. J. Plast. 117, 71-92.

Kondori, B., Benzerga, A.A., 2014. Effect of stress triaxiality on the flow and fracture of Mg alloy AZ31. Metall. Mater. Trans. 45, 3292-3307.

Krieg, R.D., 1975. A practical two surface plasticity theory. J. Appl. Mech. 42, 641-646.

Lebensohn, R.A. and Tomé, C.N., 1993. A self-consistent anisotropic approach for the simulation of plastic deformation and texture development of polycrystals: application to zirconium alloys. Acta Metall. Mater. 41(9), 2611-2624.

Lee, Y.S., Kwon, Y.N., Kang, S.H., Kim, S.W., Lee, J.H., 2008. Forming limit of AZ31 alloy sheet and strain rate on warm sheet metal forming. J. Mater. Process. Tech. 201, 431-435.

Lide, D.R., 2005. Physical Constant of Inorganic Compound. Handbook of Chemistry and Physics, 474.

Lévesque, J., Mohammadi, M., Mishra, R.K., Inal, K., 2016. An extended Taylor model to simulate localized deformation phenomena in magnesium alloys. Int. J. Plast. 78, 203-222.

Lee, M.-G., Wagoner, R.H., Lee, J.K., Chung, K., Kim, H.Y., 2008. Constitutive modeling for anisotropic/asymmetric hardening behavior of magnesium alloy sheets. Int. J. Plast. 24(4), 545-582.

Lee, J., Kim, S.-J., Lee, Y.-S., Lee, J.-Y., Kim, D., Lee, M.-G., 2017. Distortional hardening concept for modeling anisotropic/asymmetric plastic behavior of AZ31B magnesium alloy sheets. Int. J. Plast. 94, 74-97.

Li, H., Zhang, H.Q., Yang, H., Fu, M.W., Yang, H., 2017. Anisotropic and asymmetrical yielding and its evolution in plastic deformation: Titanium tubular materials. Int. J. Plast. 90, 177-211.

Lou, X.Y., Li, M., Boger, R.K., Agnew, S.R., Wagoner, R.H., 2007. Hardening evolution of AZ31B Mg sheet. Int. J. Plast. 23, 44-86.

Marciniak, Z. and Kuczyński, K., 1967. Limit strains in the processes of stretch-forming sheet metal. Int. J. Mech. Sci. 9(9), 609-620.

Madi, Y., Benzerga, A.A., Besson, J., 2017. Modeling the 3D plastic anisotropy of magnesium AZ31B alloy. In: Floryan, J.M. (Ed.), Contributions to the Foundations of Multidisciplinary Research in Mechanics, Proceedings of the XXIV International Congress of Theoretical and Applied Mechanics (ICTAM). 2730-2731.

Moiseyev, V.N., 2006. Titanium Alloys. Russian Aircraft and Aerospace Application.

Mróz, Z., 1967. On the description of anisotropic workhardening. J. Mech. Phys. Solid. 15, 163-175. 
Muhammad, W., Mohammadi, M., Kang, J., Mishra, R.K., Inal, K., 2015. An elasto-plastic constitutive model for evolving asymmetric/anisotropic hardening behavior of AZ31B and ZEK100 magnesium alloy sheets considering monotonic and reverse loading paths. Int. J. Plast. 70, 30-59.

Neilsen, M.K. and Schreyer, H.L., 1993. Bifurcations in elastic-plastic materials. Int. J. Solids Struct. 30(4), $521-544$.

Nixon, M.E., Cazacu, O., Lebensohn, R.A., 2010. Anisotropic response of high-purity $\alpha$-titanium: Experimental characterization and constitutive modeling. Int. J. Plast. 26(4), 516-532.

Plunkett, B., Lebensohn, R.A., Cazacu, O., Barlat, F., 2006. Anisotropic yield function of hexagonal materials taking into account texture development and anisotropic hardening. Acta Mater. 54(16), 4159-4169.

Paramatmuni, C., Kanjarla, A.K., 2019. A crystal plasticity FFT based study of deformation twinning, anisotropy and micromechanics in HCP materials: Application to AZ31 alloy. Int. J. Plast. 113, 269-290.

Rudnicki, J.W. and Rice, J.R., 1975. Conditions for the localization of deformation in pressure-sensitive dilatant materials. J. Mech. Phys. Solids 23(6), 371-394.

Rice, J.R., 1976. The localization of plastic deformation. In: 14th International Congress of Theoretical and Applied Mechanics, 207-220.

Sidoroff, F. and Dogui, A., 2001. Some issues about anisotropic elasticplastic models at finite strain. Int. J. Solids Struct. 38(52), 9569-9578.

Steglich, D., Tian, X., Besson, J., 2016. Mechanism-based modelling of plastic deformation in magnesium alloys. Eur. J. Mech. -A/Solids 55, 289-303.

Steglich, D., Jeong, Y., 2016. Texture-based forming limit prediction for Mg sheet alloys ZE10 and AZ31. Int. J. Mech. Sci. 117, 102-435.

Song, D. and Castañeda, P.P., 2018. Fully optimized second-order homogenization estimates for the macroscopic response and texture evolution of low-symmetry viscoplastic polycrystals. Int. J. Plast. 110, 272293.

Valanis, K.C. 1989. Banding and stability in plastic materials. Acta Mech. 79(1-2), 113-141.

Wang, H., Wu, P.D., Gharghouri, M.A., 2010. Effects of basal texture on mechanical behaviour of magnesium alloy AZ31B sheet. Mater. Sci. Eng. A 527(15), 3588-3594.

Wang, H., Wu, P.D., Boyle, K.P., Neale, K.W., 2011. On crystal plasticity formability analysis for magnesium alloy sheets. Int. J. Solids Struct. 48(6), 1000-1010.

Wu, P.D., MacEwen, S.R., Lloyd, D.J., Neale, K.W., 2004. Effect of cube texture on sheet metal formability. Mater. Sci. Eng. A 364(1-2), 182-187.

Wu, S.H., Song, N.N., Pires, F.M.A., Santos, A.D., 2015. Prediction of forming limit diagrams for materials with HCP structure. Acta Metall. Sin. 28(12), 1442-14.

Yoon, J., Cazacu, O., Mishra, R.K., 2013. Constitutive modeling of AZ31 sheet alloy with application to axial crushing. Mat. Sci. Eng. A 565, 203-212. 
Zajkani A., Bandizaki A., 2017. An efficient model for diffuse to localized necking transition in rate-dependent bifurcation analysis of metallic sheets. Int. J. Mech. Sci. 133, 794-803.

$-48-$ 